THE ASTROPHYSiCAL Journal SupPlement SERIES, 91:553-581, 1994 April

(C) 1994. The American Astronomical Society. All rights reserved. Printed in U.S.A.

\title{
EVOLUTIONARY MODELS FOR HIGH-METALLICITY GIANT EXTRAGALACTIC H II REGIONS
}

\author{
Maria Luisa García Vargas and ANGeles I. DíaZ \\ Departamento de Física Teórica, Universidad Autónoma de Madrid, 28049-Madrid, Spain \\ Received 1993 March 2; accepted 1993 August 4
}

\begin{abstract}
We present theoretical models for giant $\mathrm{H}$ II regions of high metallicity representative of those observed in the inner parts of spiral galaxies.

The regions are ionized by young star clusters whose spectral energy distributions are computed on the basis of evolutionary synthesis. The emergent ionizing continua are used as input for the photoionization code CLOUDY to obtain the corresponding emission-line spectra that are then compared to existing observations.

The possible implications on the IMF parameter determination for this kind of $\mathrm{H}$ II regions are discussed.

Subject headings: galaxies: ISM - H II regions — ISM: abundances
\end{abstract}

\section{INTRODUCTION}

The motivation of this work is to study high-metallicity starforming regions, in order to derive chemical properties of the interstellar medium and to obtain information about the physical processes which produce the ionization in such regions. Extraordinary laboratories for such a project are the extragalactic $H$ II regions, characterized by their huge size $(D \approx 500$ $\mathrm{pc}$ ), as measured from $\mathrm{H} \alpha$ images (van der Hulst et al. 1988), low density $\left(\approx 10-100 \mathrm{~cm}^{-3}\right)$, and a number of ionizing photons, derived from the observed $\mathrm{H} \alpha$-emission luminosity, of about $10^{51}-10^{52} \mathrm{~s}^{-1}$, which imply ionization by a star cluster. These regions are very different from many of the $\mathrm{H}$ il regions in our own Galaxy, smaller and probably ionized by a single star. Some reviews of the general properties of giant extragalactic $\mathrm{H}$ II regions (GEHRs) can be found in Shields (1990) and Kennicutt (1991). When these regions are located in nearby galaxies, they offer an exceptional opportunity to study the ionization structure of a giant complex of ionized gas. Some of them allow a detailed study with spatial resolution. This is the case of NGC 5471 in M101 (Skillmann 1985), 30 Dor in the LMC (Mathis, Chu, \& Peterson 1985), and NGC 604 in M33 (Díaz et al. 1987).

From the theoretical point of view, McGaugh (1991) has presented the results of applying a grid of photoionization models for $\mathrm{H}$ II regions of several metallicities, using as ionizing source the main-sequence $\mathrm{O}-\mathrm{B}$ stars of the zero-age main sequence (ZAMS) given by Maeder (1990). However, these models do not take into account the evolution of the cluster, an important factor which affects the shape of the ionizing spectrum, and consequently, the emission-line spectrum of the surrounding gas.

Díaz et al. (1991) have calculated a grid of simple photoionization models in order to investigate the ionization properties of high-metallicity $\mathrm{H}$ II regions, assuming as ionizing spectrum the spectral energy distribution (SED) of single stars. The emission-line spectra of these $H$ II regions are shown to be controlled by the shape of the ionizing radiation, the ratio of the ionizing photon density to the particle density, and the chemical composition of the gas. Therefore, from the func- tional point of view, $\mathrm{H}$ II regions form a three-parameter family characterized by the effective temperature of the ionizing stars $\left(T_{\text {eff }}\right)$, the ionization parameter $(u)$, and the metallicity $(Z)$. The work presented here tries to give a physical meaning to these models, passing from these "functional parameters": $T_{\text {eff }}, u$, and $Z$ to the corresponding star cluster parameters: age, total mass, and metallicity, assuming an evolving star cluster as the ionizing source.

Some physical properties of the ionizing star clusters can be inferred by comparing the results of these models with observational data.

\section{EVOLUTIONARY MODELS}

The models used in this work are part of a bigger grid of evolutionary models at solar metallicity, and the details of the synthesis code will be discussed in a forthcoming paper. The following two subsections give the main properties of the ionizing star clusters and their corresponding SEDs.

\subsection{Ionizing Star Clusters}

The ionizing clusters have been assumed to form in a single burst with a power-law IMF, $\phi(m)=m^{-\alpha}$, with $\alpha=2.35$ (Salpeter), 2.8, 3.35, considering the masses of formed stars between the lower limit, $m_{\text {low }}=0.85 M_{\odot}$, and the upper limit, $m_{\text {up }}=40,60,85,120 M_{\odot}$. Models are computed for total cluster masses, $M_{T}=\int_{m_{\text {low }}}^{m_{\text {up }}} m \phi(m) d m$, between $0.12 \times 10^{5} M_{\odot}$ and $2 \times 10^{6} M_{\odot}$. Clusters in this range of masses, with an appropriate IMF, can provide the necessary ionizing photons, $Q(\mathrm{H})$ to produce the observed $\mathrm{H} \alpha$ luminosities $[\log Q(\mathrm{H}) \approx$ 51]. The grid of computed models is summarized in Table 1. Additional models for $\alpha=1.7$ and $M_{T}=0.1 \times 10^{5} M_{\odot}$ have been computed for selected ages in order to show extreme effects in the SEDs ( see $\S 2.2$ ).

The clusters are evolved along the evolutionary tracks of Maeder \& Meynet (1989) for stars between 0.85 and $120 M_{\odot}$ and solar composition. These tracks are computed taking into account mass loss and moderate core overshooting. Isochrones for different ages between 1.5 and 5.4 Myr have been com- 
TABLE 1

IONIZING CLUSTER PARAMETERS

\begin{tabular}{lrl}
\hline \hline \multicolumn{1}{c}{$\alpha$} & $m_{\text {up }}$ & \multicolumn{1}{c}{$M_{T}\left(\times 10^{5} M_{\odot}\right)$} \\
\hline $2.35 \ldots \ldots$ & 120 & $0.12,0.2,0.4,0.6$ \\
$2.35 \ldots \cdots$ & 60 & $0.4,0.6$ \\
$2.35 \ldots \ldots$ & 40 & $0.4,0.6$ \\
$2.80 \ldots \ldots$ & 120 & $0.4,0.6,4$ \\
$3.35 \ldots \ldots$ & 120 & $4,6,8,20$ \\
$3.35 \ldots \ldots$ & 60 & 20 \\
$3.35 \ldots \cdots$ & 40 & 20 \\
\hline
\end{tabular}

puted by interpolation between points of adjacent tracks corresponding to the same evolutionary phase. Clusters older than 5.4 Myr cannot provide the ionizing photons required to reproduce the observations.

Stars more massive than $40 M_{\odot}$ start their evolution to the left of the H-R diagram at about $3 \mathrm{Myr}$ and appear as extreme W-R stars between 3.5 and 5.4 Myr. These stars, if present, dominate the cluster ionizing spectrum, due to their high effective temperature and luminosity. At about $4 \mathrm{Myr}$, the precursors of these stars go through a rapid red supergiant phase. Since the mass-spectrum has been generated with an analytical power law for the IMF, for the less massive clusters only a small number (or even none) of these stars are present. The number of massive stars depends on $M_{T}$ and the IMF parameters and decreases for lower values of $\alpha$, higher values of $m_{\mathrm{up}}$, or both. This richness effect is presented in Table 2, in which the number of extreme W-R stars is given for clusters synthesized with different masses and IMF parameters.

\subsection{Spectral Energy Distributions}

For each of the clusters in each evolutionary stage, we have synthesized the emergent spectrum by calculating the number of stars in each point of the H-R diagram and assigning to it the most adequate stellar atmosphere model (the closest one in effective temperature and surface gravity). The corresponding stellar spectrum has then been scaled to the luminosity of the corresponding theoretical star in the H-R diagram. The atmosphere models we have used are those of Clegg \& Middlemass (1987) for stars with $T_{\text {eff }} \geq 50,000 \mathrm{~K}$ (corresponding to the last evolutionary stages of massive stars) and those of Kurucz (1979) for stars with $5500 \mathrm{~K} \leq T_{\text {eff }}<50,000 \mathrm{~K}$. For stars with $T_{\text {eff }}<5500 \mathrm{~K}$, we have used the stellar atlas of Jacobi, Hunter, \& Christian (1984). We have chosen Kurucz's models for two reasons: first, because there is a good agreement between the models of Kurucz and Clegg \& Middlemass of a same $T_{\text {eff }}$, which provides a consistent temperature scale, and second, because Kurucz's models include blanketing effects, which can be important at high metallicity.

Figure 1 shows the synthesized spectra of a cluster of $0.6 \times$ $10^{5} M_{\odot}$ with the "standard" IMF $\left(\alpha=2.35, m_{\text {low }}=0.85 M_{\odot}\right.$, $m_{\mathrm{up}}=120 M_{\odot}$ ) for different ages from 2 to $5.5 \mathrm{Myr}$. The spectrum becomes harder at 3.5 and $4 \mathrm{Myr}$ due to the appearance of the extreme W-R stars. Nevertheless, the optical spectra are almost identical for all ages except around $4 \mathrm{Myr}$ where the contribution of red supergiants becomes conspicuous.

For a given cluster, the number of Lyman ionizing photons, $Q(\mathrm{H})$, does not change much during its evolution; however, the ratio of the number of helium ionizing photons, $Q(\mathrm{He})$, to $Q(\mathrm{H})$ changes appreciably. We have defined an equivalent ionizing temperature, $T_{\text {eq }}$, for each cluster as $T_{\text {eff }}$ of an ionizing star whose spectrum has the same value of $Q(\mathrm{He}) / Q(\mathrm{H})$. According to this definition, the computed cluster spectra can reach temperatures between $40,000 \mathrm{~K}$ and $60,000 \mathrm{~K}$.

For a given age, clusters with higher mass have higher lumin-

TABLE 2

Number of EXTREME WOLF-RAYET STARS AS A FunCTION OF CLUSTER PARAMETERS A. $\alpha=2.35, m_{\mathrm{up}}=120 M_{\odot}$

\begin{tabular}{ccccc}
\hline $\begin{array}{c}\text { Age } \\
(\mathrm{Myr})\end{array}$ & $\begin{array}{c}0.1 \times 10^{5} \\
M_{\odot}\end{array}$ & $\begin{array}{c}0.12 \times 10^{5} \\
M_{\odot}\end{array}$ & $\begin{array}{c}0.2 \times 10^{5} \\
M_{\odot}\end{array}$ & $\begin{array}{c}0.6 \times 10^{5} \\
M_{\odot}\end{array}$ \\
\hline $3.5 \ldots \ldots$ & 0 & 1 & 5 & 20 \\
$4.0 \ldots \ldots$ & 2 & 3 & 6 & 23 \\
$4.5 \ldots \ldots$ & 3 & 3 & 7 & 21 \\
$5.4 \ldots \ldots$ & 3 & 4 & 6 & 20 \\
\hline
\end{tabular}

B. $\alpha=2.80, m_{\mathrm{up}}=120 M_{\odot}$

\begin{tabular}{ccccc}
\hline $\begin{array}{c}\text { Age } \\
(\mathrm{Myr})\end{array}$ & $\begin{array}{c}0.6 \times 10^{5} \\
M_{\odot}\end{array}$ & $\begin{array}{c}4 \times 10^{5} \\
M_{\odot}\end{array}$ & $\begin{array}{c}6 \times 10^{5} \\
M_{\odot}\end{array}$ & $\begin{array}{c}8 \times 10^{5} \\
M_{\odot}\end{array}$ \\
\hline $3.5 \ldots \ldots$ & 3 & 31 & 50 & 66 \\
$4.0 \ldots \ldots$ & 5 & 44 & 66 & 89 \\
$4.5 \ldots \ldots$ & 5 & 34 & 65 & 88 \\
$5.4 \ldots \ldots$ & 7 & 47 & 70 & 94 \\
\hline
\end{tabular}

\begin{tabular}{ccccc}
\hline \multicolumn{5}{c}{ C. $\alpha=3.35, m_{\text {up }}=120 M_{\odot}$} \\
\hline \hline Age & $0.4 \times 10^{5}$ & $0.6 \times 10^{5}$ & $4 \times 10^{5}$ & $8 \times 10^{5}$ \\
$(\mathrm{Myr})$ & $M_{\odot}$ & $M_{\odot}$ & $M_{\odot}$ & $M_{\odot}$ \\
\hline $3.5 \ldots \ldots$ & 0 & 0 & 1 & 8 \\
$4.0 \ldots \ldots$ & 0 & 0 & 5 & 11 \\
$4.5 \ldots \ldots$ & 0 & 0 & 7 & 14 \\
$5.4 \ldots \ldots$ & 0 & 1 & 9 & 18 \\
\hline
\end{tabular}

D. $\alpha=3.35, m_{\mathrm{up}}=40 M_{\odot}$

\begin{tabular}{ccccc}
\hline \hline Age & $4 \times 10^{5}$ & $6 \times 10^{5}$ & $8 \times 10^{5}$ & $2 \times 10^{5}$ \\
$(\mathrm{Myr})$ & $M_{\odot}$ & $M_{\odot}$ & $M_{\odot}$ & $M_{\odot}$ \\
\hline
\end{tabular}

\begin{tabular}{lllll}
$3.5 \ldots \ldots$ & 0 & 0 & 0 & 0 \\
$4.0 \ldots \ldots$ & 0 & 0 & 0 & 0 \\
$4.5 \ldots \ldots$ & 0 & 0 & 0 & 0 \\
$5.4 \ldots \ldots$ & 0 & 0 & 0 & 0 \\
\hline
\end{tabular}

E. EFFECT OF $m_{\mathrm{up}}$ FOR A CLUSTER WITH $M_{T}=0.4 \times 10^{5} M_{\odot}$

\begin{tabular}{lccccc}
\hline \hline $\begin{array}{c}\text { Age } \\
(\mathrm{Myr})\end{array}$ & $40 M_{\odot}$ & $60 M_{\odot}$ & $85 M_{\odot}$ & $100 M_{\odot}$ & $120 M_{\odot}$ \\
\hline $3.5 \ldots \ldots$ & 0 & 0 & 3 & 7 & 12 \\
$4.0 \ldots \ldots$ & 0 & 2 & 15 & 15 & 15 \\
$4.5 \ldots \ldots$ & 0 & 14 & 14 & 14 & 14 \\
$5.4 \ldots \ldots$ & 0 & 13 & 13 & 13 & 13 \\
\hline
\end{tabular}




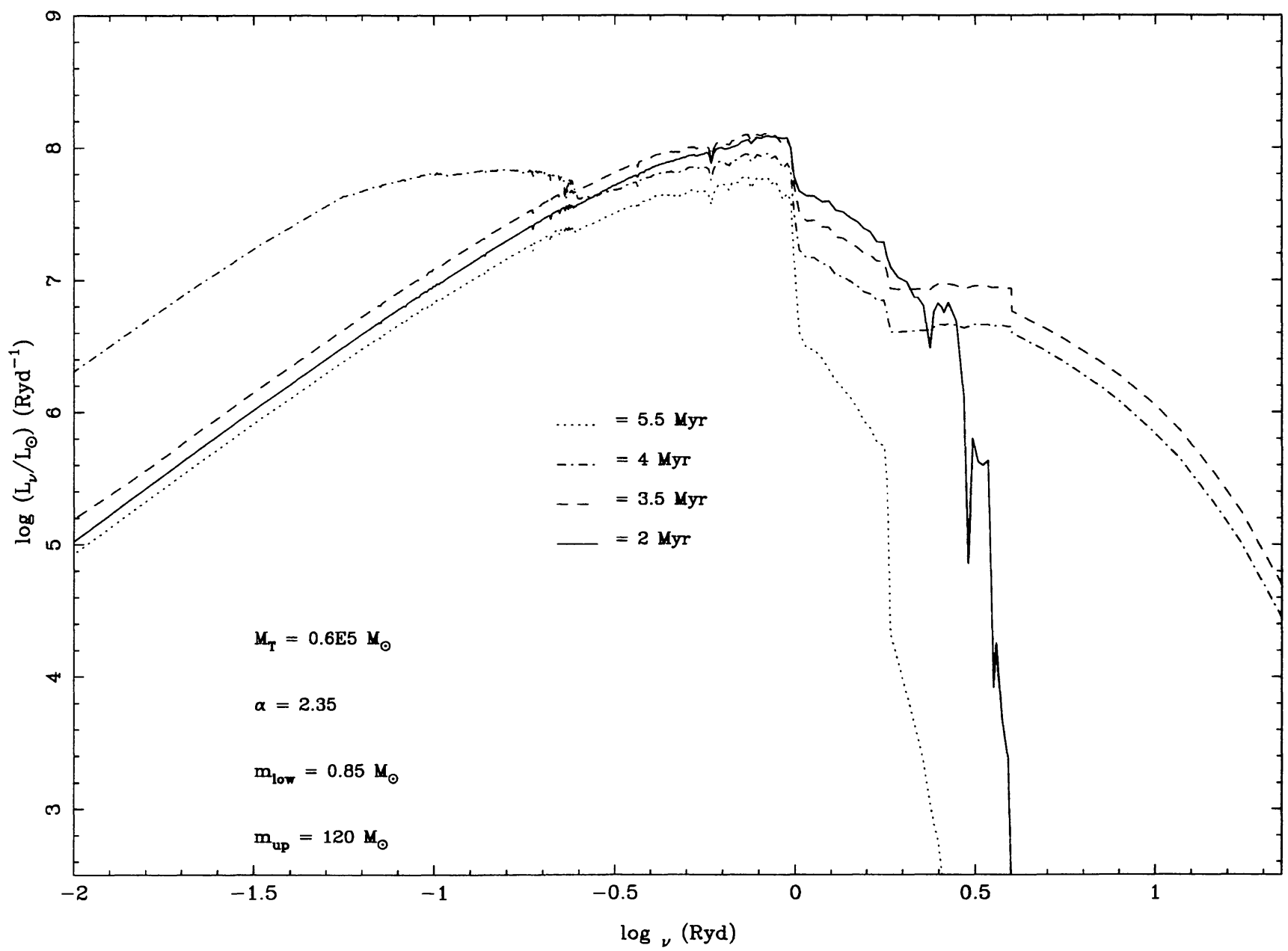

F1G. 1.-Evolution of the spectral energy distribution for a cluster with $M_{T}=0.6 \times M_{\odot}$ synthesized with the standard IMF

osities as can be seen from Figure 2, in which we represent the synthesized spectra for clusters with different masses, at an age of $3.5 \mathrm{Myr}$, for standard values of the IMF as above. We can see the "richness effect": for the less massive cluster $\left(M_{T}=1 \times\right.$ $10^{4}$ ), we see a cutoff in the spectrum at 1 ryd, due to the absence of extreme Wolf-Rayet stars, which are not formed.

The importance of the IMF parameters in the SED of the cluster can be seen in Figures 3 and 4 which show spectra for a 4 Myr old cluster with $M_{T}=0.4 \times 10^{5} M_{\odot}$, in which the IMF parameters, $\alpha$ in Figure 3 and $m_{\mathrm{up}}$ in Figure 4, are allowed to vary. It can be seen that for certain values of these parameters there is a cutoff in the shape of the ionizing spectrum beyond 1 ryd due to the lack of extreme W-R stars.

\section{PHOTOIONIZATION MODELS}

The cluster ionizing spectra computed as described in the previous section have been used as input for the photionization code CLOUDY (Ferland 1990). The resulting emissionline spectra of the ionized regions have been computed, under certain hypotheses about the geometry and the physical and chemical conditions of the gas. A static spherical geometry is assumed in the models, considering the star cluster in the center of the sphere, and the gas surrounding it, located in a shell at an inner radius $R$ from the ionizing source. Constant density throughout the nebula has been assumed for simplicity. The ionization parameter, $u$, is defined as the ratio between the density of the ionizing photons and the density of particles, and is equal to $u=Q(\mathrm{H}) / 4 \pi c n_{\mathrm{H}} R^{2}$. The density of the ionizing photons is calculated from the total number of Lyman-continuum photons ( $\nu \geq 1$ ryd) which reach the region of radius $R$ at a velocity, $c$, and $n_{\mathrm{H}}$ is the density of ionized hydrogen, taken to be equal to the electronic density, $n_{e}$, if the region is completely ionized. Contributions to $n_{e}$ from ionized He amount to only $10 \%$ in the more excited regions. For our models the assumed values for $n_{\mathrm{H}}$ and $R$ are $n_{\mathrm{H}}=10 \mathrm{~cm}^{-3}$ and $R=250 \mathrm{pc}$, values characteristic of extragalactic $\mathrm{H}$ II regions (e.g., van der Hulst et al.; 1988, Díaz et al. 1991) which, together with the number of ionizing photons corresponding to each cluster, $Q(\mathrm{H})$, determine the ionization parameter, $u$. This ionization parameter changes by a factor of about 2 during the evolution of a given cluster. 


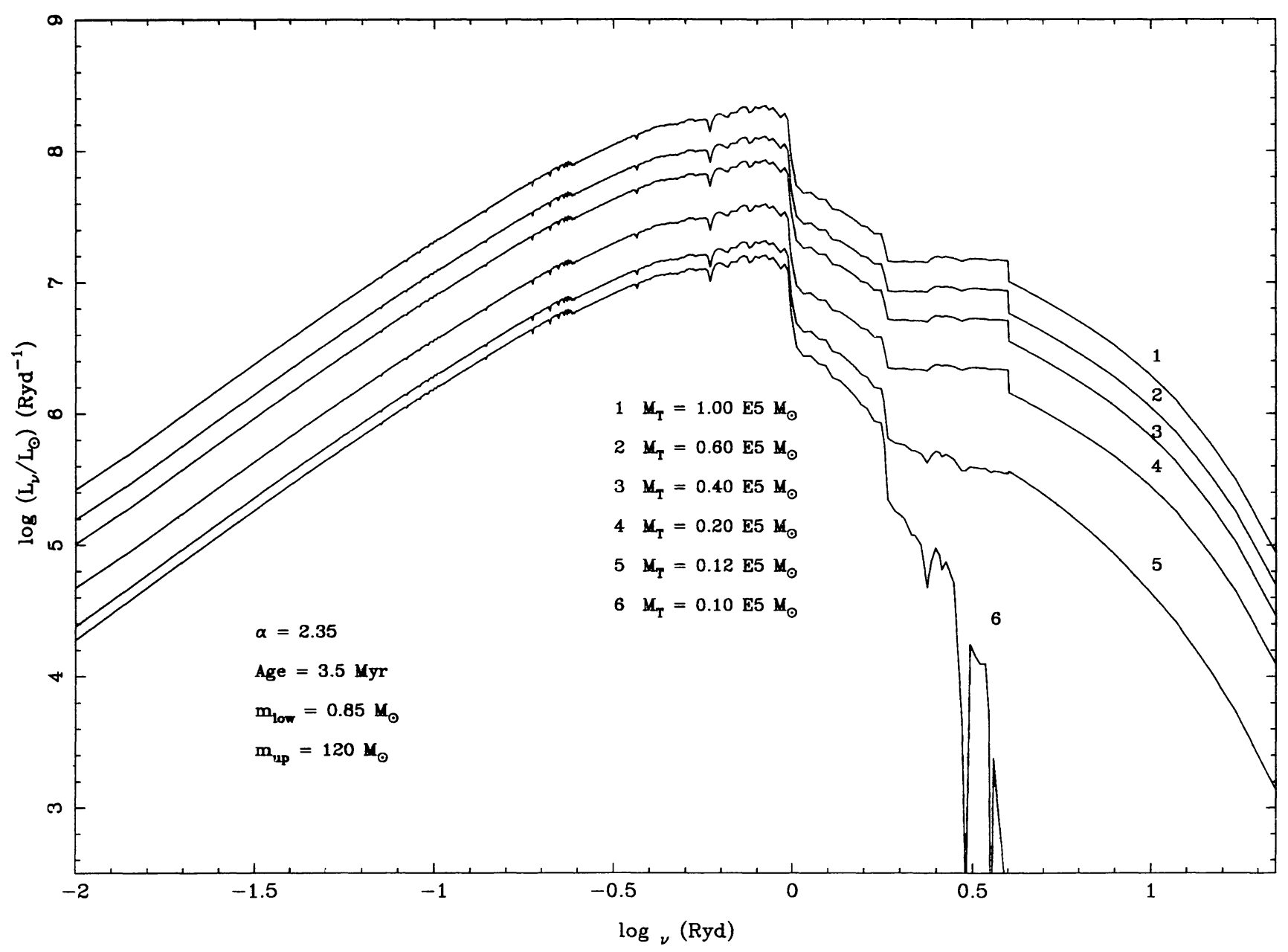

FIG. 2.-Richness effect in the spectral energy distribution for 3.5 Myr clusters with different masses

For a given IMF, the emission-line spectrum is controlled ( $a$ ) by the ionization parameter that changes mainly according to the mass of the cluster through the value of $Q(\mathrm{H}) ;(b)$ by the shape of the ionizing continuum that changes with the age of the cluster; and $(c)$ by the metallicity of the gas, which we have taken to be either solar or twice solar. Table 3 presents the solar abundances as assumed in our set of models. All of the values are the same as in Stasińska (1990) (including $\mathrm{Ca}, \mathrm{Mg}, \mathrm{Si}$, and $\mathrm{Fe}$, which were depleted by a factor of 10 ), except the sulfur abundance, for which the ratio $\mathrm{S} / \mathrm{O}$ given by Grevesse \& Anders (1989) has been taken. The twice solar metallicity has all the solar abundances multiplied by a factor of 2 , except for helium.

Some limitations of these models should be mentioned. First, the hypothesis of constant density through the nebula might not be the most adequate. There are observations in some objects with emission-line spectra implying a correlation between the equivalent widths of forbidden lines and the upper levels from which these lines may be collisionally deexcited (De Robertis \& Osterbrock 1986), which can be explained only if the photoionized gas presents a certain density gradient.
Models which include this effect (Stasińska 1984; Péquignot 1984; Binette 1985; Ferland \& Osterbrock 1986) yield abundances of some metallic elements lower than those obtained for models with constant density. Since these latter models assume maximum efficiency for the emission in lines which get collisionally deexcited, the conditions would be less favorable if a stratification in density is assumed.

Second, these models do not include the effects of shock waves probably generated in supernova explosions: SN Type Ib subluminous, whose progenitors are stars with masses between 25 and $120 M_{\odot}$ and explode between 3.5 and $10 \mathrm{Myr}$, and SN Type II, final stages of stars with masses between 8 and $25 M_{\odot}$ exploding after 5.5-50 Myr. The noninclusion of these effects can lead to an underestimation of some emission lines. The treatment of this contribution is very complex, and a first solution to the problem is discussed in Terlevich et al. (1992). This is the reason for stopping our calculations at ages later than 5.4 Myr, since clusters older than this age cannot provide the ionizing photons required to reproduce the observations of GEHRs without including the effects of SN explosions.

Third, some amount of dust is expected to be associated with 


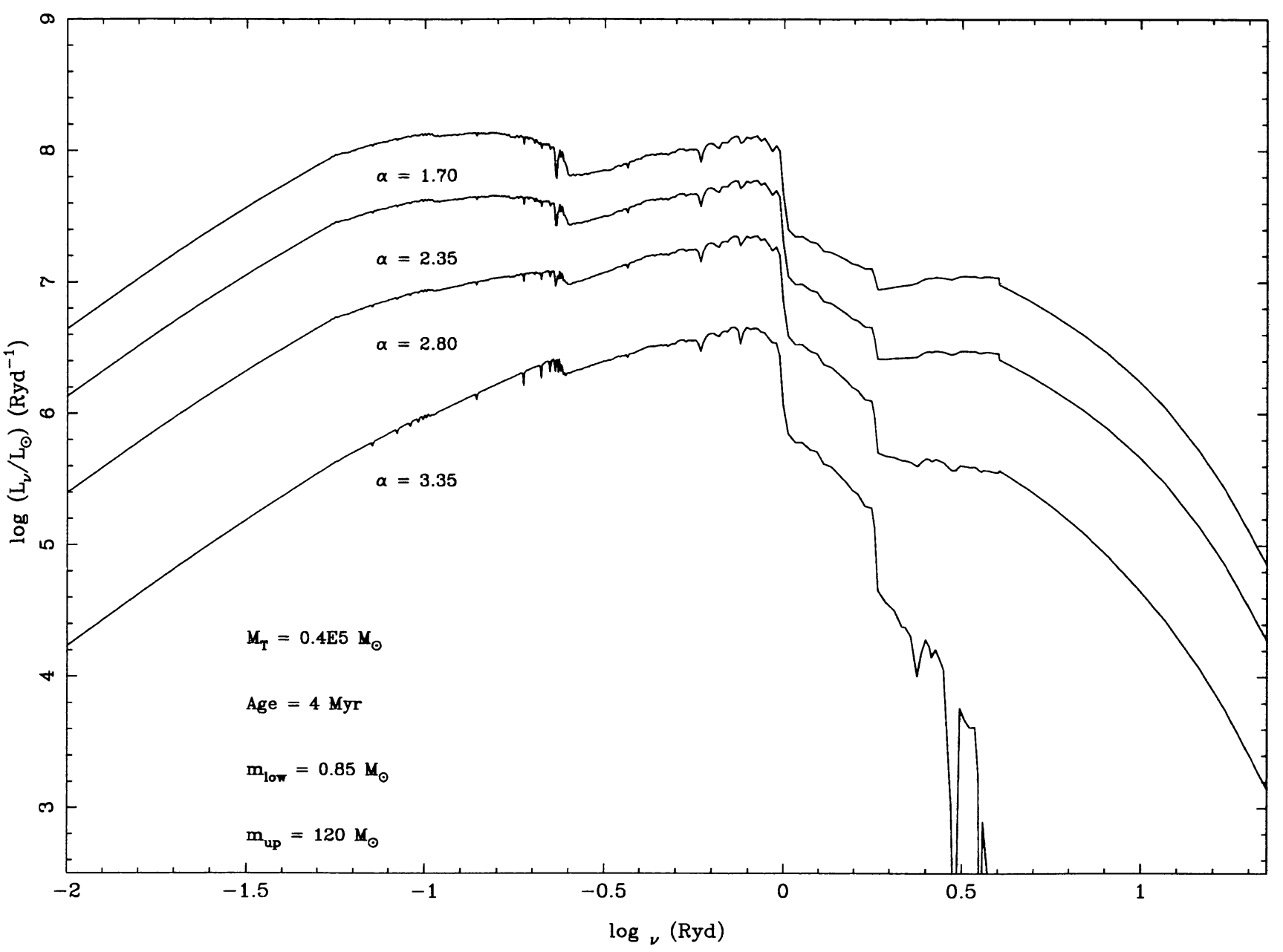

FIG. 3.-Effect in the spectral energy distribution of the power-law index of the IMF, $\alpha$, variation

the cluster, and this effect has not been considered in this set of models, except for a depletion of the heavy elements, as can be seen in the assumed abundances. The most important effect would be the absorption of part of the Lyman-continuum photons by this dust, which would be reemitted in the IR. This fact implies that our estimations of the cluster masses are only lower limits.

\section{RESULTS}

The results of the models are presented in Tables 4-20. Each ionizing cluster is characterized by its total mass, $M_{T}$, age, and IMF parameters $\left(\alpha, m_{\mathrm{low}}\right.$, and $\left.m_{\mathrm{up}}\right)$ as given in Table 1 . These tables include information about the intrinsic properties of the ionizing cluster, and the emission-line spectrum of the gas, assuming solar and twice solar abundances. Each table has five parts, marked a, b, c, d, and e.

The tables lettered (a) include the following information: Columns (1)-(5) present intrinsic properties of the ionizing clusters, which are independent of both the assumed geometry and the metallicity of the gas. These properties are (1) age of the cluster (Myr); (2) $\log L_{\mathrm{Ly} \alpha}$, logarithm of the ionizing luminosity, in ergs $^{-1} ;(3) \log [Q(\mathrm{H})]$, logarithm of the total number of ionizing photons ( $\nu \geq 1$ ryd) per second; (4) $Q(\mathrm{He}) /$ $Q(\mathrm{H})$, ratio between the number of helium ionizing photons $(\nu \geq 1.8 \mathrm{ryd})$ and the total ionizing photons; $(5) Q(\mathrm{O}) / Q(\mathrm{H})$, ratio of the number of $\mathrm{O}^{+}$ionizing photons $(\nu \geq 2.6 \mathrm{ryd})$ to the total ionizing photons. Columns (6)-(9) present some properties independent of the metallicity, but affected by the geometry, the radius, $R$, and the gas density. These parameters are (6) $\log u ;(7) \log I(\mathrm{H} \beta)$, in emission $\left(\mathrm{ergs}^{-1}\right) ;(8) \epsilon$, the filling factor, given by the ratio $\epsilon=\left(r_{1} / r\right)^{3}$, where $r$ is the observed radius, and $r_{1}$ is the Strömgrem radius, defined as $r_{1}=$ $\left[3 Q(\mathrm{H}) / 4 \pi \alpha_{\mathrm{B}} \mathrm{n}_{\mathrm{H}}^{2}\right]^{1 / 3}$, where $\alpha_{\mathrm{B}}$ is the case $\mathrm{B}$ recombination coefficient; and (9), the total mass of ionized hydrogen in solar masses, calculated with the expression $M_{\mathrm{H} \text { II }} / M_{\odot}=5.58 \times 10^{13}$ $u^{3} \epsilon^{-2} n_{\mathrm{H}}^{-2}$.

The tables lettered $(b)$ and $(c)$ (at solar abundances) and $(d)$ and $(e)$ (at twice solar abundances) present the intensity ratios, $I_{\lambda} / I(\mathrm{H} \beta)$, of some important emission lines of the gas, ionized by the cluster in each evolutionary stage. All of the line wavelengths are in $\AA$, except for [C II] which is given in microns. 


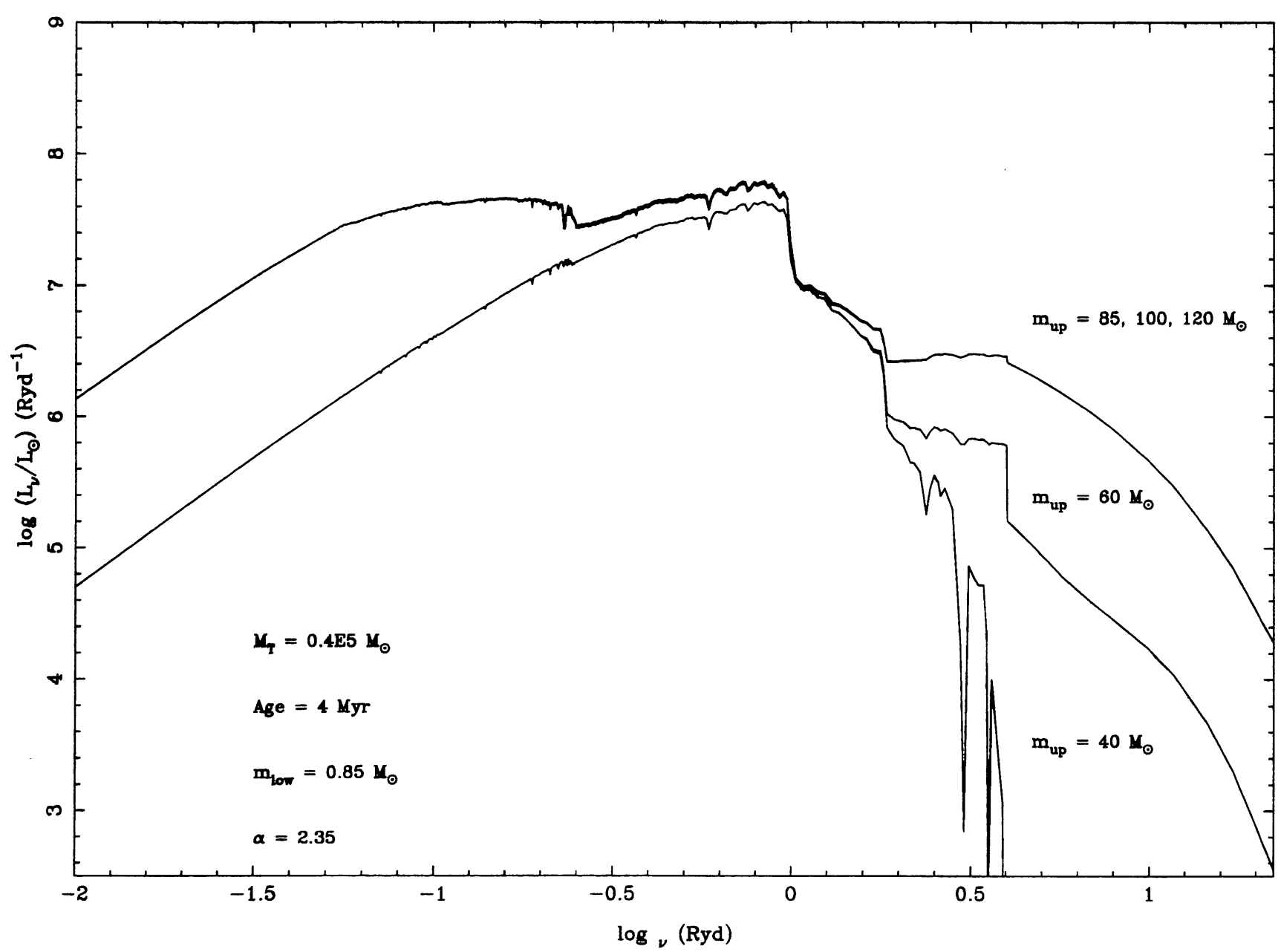

FIG. 4.-Effect in the spectral energy distribution of the upper mass limit of the IMF, $m_{\mathrm{up}}$, variation

The last two columns in the tables lettered $(c)$ and $(e)$ give the electron temperature, $T_{e}$, (in CLOUDY $T_{p}$, defined as the mean electron temperature, weighted by the $\mathrm{H}^{+}$density) and the equivalent width of the $\mathrm{H} \beta$ line in $\AA$, which has been calculated considering both nebular and cluster continua.

TABLE 3

ASSUMED SOLAR ABUNDANCES

\begin{tabular}{|c|c|}
\hline$Z_{i}$ & $\log \left(Z_{i} / \mathrm{H}\right)$ \\
\hline $\mathrm{He} .$. & -1.000 \\
\hline $\mathrm{C} \ldots \ldots \ldots \ldots$ & -3.328 \\
\hline $\mathrm{N} \ldots \ldots \ldots \ldots \ldots$ & -4.009 \\
\hline $\mathrm{O} \ldots \ldots \ldots \ldots$ & -3.081 \\
\hline $\mathrm{Ne} \ldots \ldots \ldots \ldots \ldots$ & -3.959 \\
\hline $\operatorname{Mg} \ldots \ldots \ldots \ldots$ & -5.585 \\
\hline $\mathrm{Al} \ldots \ldots \ldots \ldots \ldots$ & -5.569 \\
\hline $\mathrm{Si} \ldots \ldots \ldots \ldots \ldots$ & -5.398 \\
\hline$S \ldots \ldots \ldots \ldots+\cdots$ & -4.782 \\
\hline $\operatorname{Ar} \ldots \ldots \ldots \ldots$ & -5.201 \\
\hline $\mathrm{Ca} \ldots \ldots \ldots \ldots \ldots$ & -5.638 \\
\hline $\mathrm{Fe} \ldots \ldots \ldots \ldots \ldots$ & -5.796 \\
\hline
\end{tabular}

\section{DISCUSSION}

It can be seen from these tables that all the models synthesized with a standard IMF have $\mathrm{H} \beta$ equivalent widths between 64 and $610 \AA$. These values are similar to those calculated by other authors with different synthesis models (Melnick, Terlevich, \& Eggleton 1985; Mas-Hesse \& Kunth 1991). However, most of the observed $\mathrm{H}$ II regions of high metallicity have values of $\mathrm{EW}(\mathrm{H} \beta)$ between 5 and $90 \AA$. One way of reproducing such small equivalent widths is to hypothesize the presence of an underlying older stellar population which would provide an extra continuum. The existence of such a population may be difficult to explain in the arms of spiral galaxies where many of these regions are located. A more plausible way out is to consider the ionizing cluster as composed of smaller subregions which are seen as a single one due to the lack of spatial resolution. In fact the few spatially resolved observations of GEHR show several emitting regions with very different spectra (Skillman 1985; Díaz et al. 1987). Very weak emission-line spectra are observed coming from the central part of the region. This might be due to the effect of the winds of the more massive stars in the cluster which have swept out the gas. The different 
TABLE 4

$M_{T}=0.12 \times 10^{5} M_{\odot}$, IMF PARAMETERS: $\alpha=2.35, m_{\mathrm{low}}=0.85 M_{\odot}, m_{\mathrm{up}}=120 M_{\odot}$

Table 4.a. Parameters independent of metallicity

\begin{tabular}{ccccccccc}
\hline \hline Age & $\log L_{L y \alpha}$ & $\log Q H$ & QHe/QH & QO/QH & $\log u$ & $\log I_{H}$ & $\epsilon$ & $M_{H I I} / \mathrm{M}_{\odot}$ \\
\hline 1.5 & 40.26 & 50.73 & 0.22 & 0.05 & -3.60 & 38.45 & 0.008 & $0.13 \mathrm{E}+06$ \\
2.0 & 40.19 & 50.70 & 0.16 & 0.03 & -3.64 & 38.41 & 0.008 & $0.12 \mathrm{E}+06$ \\
2.5 & 40.19 & 50.70 & 0.14 & 0.02 & -3.64 & 38.41 & 0.008 & $0.12 \mathrm{E}+06$ \\
3.0 & 40.15 & 50.65 & 0.15 & 0.03 & -3.68 & 38.37 & 0.007 & $0.11 \mathrm{E}+06$ \\
3.5 & 40.21 & 50.60 & 0.24 & 0.15 & -3.73 & 38.32 & 0.006 & $0.97 \mathrm{E}+05$ \\
4.0 & 40.23 & 50.52 & 0.39 & 0.27 & -3.82 & 38.26 & 0.005 & $0.76 \mathrm{E}+05$ \\
4.5 & 40.07 & 50.42 & 0.37 & 0.23 & -3.92 & 38.13 & 0.004 & $0.62 \mathrm{E}+05$ \\
5.0 & 40.18 & 50.53 & 0.41 & 0.21 & -3.80 & 38.25 & 0.005 & $0.82 \mathrm{E}+05$ \\
5.1 & 40.15 & 50.40 & 0.49 & 0.35 & -3.94 & 38.13 & 0.004 & $0.59 \mathrm{E}+05$ \\
5.2 & 39.88 & 50.13 & 0.48 & 0.35 & -4.21 & 37.86 & 0.002 & $0.31 \mathrm{E}+05$ \\
5.3 & 39.80 & 50.07 & 0.44 & 0.32 & -4.27 & 37.79 & 0.002 & $0.27 \mathrm{E}+05$ \\
5.4 & 39.69 & 49.95 & 0.43 & 0.32 & -4.39 & 37.67 & 0.001 & $0.21 \mathrm{E}+05$ \\
\hline
\end{tabular}

Table 4.b. Emission line spectrum at solar metallicity (I)

\begin{tabular}{cccccccc}
\hline \hline Age & Ly $\alpha$ 1216 & HeII 1640 & MgII 2800 & [OII] 3727 & HeII 4686 & [OIII] 5007 & [OI] 6300 \\
\hline 1.5 & 23.06 & 0.00 & 0.06 & 3.90 & 0.00 & 0.26 & 0.17 \\
2.0 & 22.99 & 0.00 & 0.04 & 3.21 & 0.00 & 0.12 & 0.11 \\
2.5 & 22.96 & 0.00 & 0.04 & 2.92 & 0.00 & 0.09 & 0.10 \\
3.0 & 22.97 & 0.00 & 0.04 & 2.99 & 0.00 & 0.10 & 0.11 \\
3.5 & 23.28 & 0.38 & 0.08 & 4.61 & 0.04 & 0.34 & 0.42 \\
4.0 & 24.14 & 0.70 & 0.15 & 7.26 & 0.08 & 0.52 & 0.88 \\
4.5 & 23.91 & 0.31 & 0.16 & 6.62 & 0.03 & 0.32 & 0.82 \\
5.0 & 23.87 & 0.32 & 0.16 & 7.05 & 0.03 & 0.47 & 0.68 \\
5.1 & 24.83 & 0.83 & 0.20 & 8.95 & 0.09 & 0.42 & 1.28 \\
5.2 & 24.76 & 0.66 & 0.23 & 8.20 & 0.07 & 0.14 & 1.63 \\
5.3 & 24.49 & 0.56 & 0.21 & 7.25 & 0.06 & 0.10 & 1.60 \\
5.4 & 24.27 & 0.42 & 0.21 & 6.47 & 0.05 & 0.06 & 1.71 \\
\hline
\end{tabular}

Table 4.c. Emission line spectrum at solar metallicity (II)

\begin{tabular}{cccccccc}
\hline \hline Age & [NII] 6584 & [SII] 6716 & [SII] 6731 & [SIII] 9532 & [CII] 158 $\mu \mathrm{m}$ & $\mathrm{EW}(\mathrm{H} \beta)$ & $T_{e}(K)$ \\
\hline 1.5 & 1.77 & 1.11 & 0.77 & 0.66 & 2.35 & 484.86 & 7140 \\
2.0 & 1.57 & 1.03 & 0.71 & 0.51 & 2.40 & 391.38 & 6880 \\
2.5 & 1.49 & 0.98 & 0.68 & 0.47 & 2.40 & 355.95 & 6760 \\
3.0 & 1.52 & 1.04 & 0.71 & 0.45 & 2.48 & 282.83 & 6790 \\
3.5 & 2.09 & 1.73 & 1.19 & 0.51 & 3.60 & 226.67 & 7290 \\
4.0 & 2.96 & 2.58 & 1.78 & 0.65 & 4.68 & 122.16 & 7790 \\
4.5 & 2.80 & 2.46 & 1.70 & 0.57 & 4.28 & 161.68 & 7770 \\
5.0 & 2.82 & 2.20 & 1.52 & 0.75 & 3.60 & 265.84 & 7910 \\
5.1 & 3.55 & 3.26 & 2.25 & 0.67 & 5.72 & 214.04 & 7970 \\
5.2 & 3.55 & 3.79 & 2.61 & 0.47 & 7.01 & 114.10 & 7830 \\
5.3 & 3.31 & 3.70 & 2.55 & 0.40 & 7.28 & 92.95 & 7650 \\
5.4 & 3.14 & 3.80 & 2.61 & 0.34 & 8.30 & 70.39 & 7440 \\
\hline
\end{tabular}

Table 4.d. Emission line spectrum at twice solar metallicity (I)

\begin{tabular}{cccccccc}
\hline \hline Age & Ly $\alpha$ 1216 & HeII 1640 & MgII 2800 & [OII] 3727 & HeII 4686 & [OIII] 5007 & [OI] 6300 \\
\hline 1.5 & 22.74 & 0.00 & 0.02 & 1.59 & 0.00 & 0.11 & 0.12 \\
2.0 & 22.69 & 0.00 & 0.01 & 1.15 & 0.00 & 0.04 & 0.07 \\
2.5 & 22.67 & 0.00 & 0.01 & 0.97 & 0.00 & 0.04 & 0.06 \\
3.0 & 22.69 & 0.00 & 0.01 & 1.04 & 0.00 & 0.04 & 0.07 \\
3.5 & 22.77 & 0.34 & 0.03 & 2.06 & 0.04 & 0.15 & 0.29 \\
4.0 & 22.91 & 0.65 & 0.07 & 4.18 & 0.07 & 0.30 & 0.72 \\
4.5 & 22.88 & 0.28 & 0.07 & 3.58 & 0.03 & 0.18 & 0.65 \\
5.0 & 22.90 & 0.29 & 0.07 & 3.93 & 0.03 & 0.26 & 0.55 \\
5.1 & 23.01 & 0.78 & 0.10 & 5.74 & 0.09 & 0.28 & 1.10 \\
5.2 & 22.94 & 0.62 & 0.10 & 4.80 & 0.07 & 0.10 & 1.25 \\
5.3 & 22.89 & 0.52 & 0.08 & 3.97 & 0.06 & 0.07 & 1.16 \\
5.4 & 22.82 & 0.38 & 0.07 & 3.26 & 0.04 & 0.04 & 1.13 \\
\hline
\end{tabular}

Table 4.e. Emission line spectrum at twice solar metallicity (II)

\begin{tabular}{cccccccc}
\hline \hline Age & [NII] 6584 & [SII] 6716 & [SII] 6731 & [SIII] 9532 & [CII] 158 $\mu \mathrm{m}$ & $\mathrm{EW}(\mathrm{H} \beta)$ & $T_{e}(K)$ \\
\hline 1.5 & 1.33 & 0.90 & 0.62 & 0.58 & 4.09 & 503.59 & 5510 \\
2.0 & 1.10 & 0.77 & 0.53 & 0.44 & 4.15 & 400.21 & 5270 \\
2.5 & 1.00 & 0.71 & 0.49 & 0.39 & 4.15 & 361.62 & 5160 \\
3.0 & 1.04 & 0.76 & 0.52 & 0.38 & 4.26 & 287.38 & 5200 \\
3.5 & 1.66 & 1.44 & 0.99 & 0.46 & 5.90 & 227.93 & 5690 \\
4.0 & 2.76 & 2.48 & 1.70 & 0.69 & 7.61 & 119.68 & 6280 \\
4.5 & 2.51 & 2.30 & 1.58 & 0.59 & 7.20 & 160.09 & 6170 \\
5.0 & 2.57 & 2.10 & 1.44 & 0.78 & 6.20 & 267.79 & 6280 \\
5.1 & 3.57 & 3.31 & 2.27 & 0.77 & 9.23 & 210.67 & 6530 \\
5.2 & 3.34 & 3.59 & 2.47 & 0.53 & 11.51 & 111.33 & 6310 \\
5.3 & 2.97 & 3.36 & 2.30 & 0.45 & 11.79 & 90.97 & 6130 \\
5.4 & 2.64 & 3.23 & 2.21 & 0.36 & 13.20 & 68.19 & 5890 \\
\hline
\end{tabular}


TABLE 5

$M_{T}=0.2 \times 10^{5} M_{\odot}$, IMF PARAMETERS: $\alpha=2.35, m_{\mathrm{low}}=0.85 M_{\odot}, m_{\mathrm{up}}=120 M_{\odot}$

Table 5.a. Parameters independent of metallicity

\begin{tabular}{ccccccccc}
\hline \hline Age & $\log L_{L y \alpha}$ & $\log Q H$ & $\mathrm{QHe}_{\mathrm{QH}}$ & $\mathrm{QO} / \mathrm{QH}$ & $\log u$ & $\log I_{H}$ & $\epsilon$ & $\bar{M}_{H I I} / \mathrm{M}_{\odot}$ \\
\hline $\mathbf{1 . 5}$ & $\mathbf{4 0 . 6 3}$ & 51.10 & 0.23 & 0.06 & -3.23 & 38.81 & 0.019 & $0.31 \mathrm{E}+06$ \\
2.0 & $\mathbf{4 0 . 5 6}$ & 51.06 & 0.16 & 0.03 & -3.28 & 38.76 & 0.017 & $0.27 \mathrm{E}+06$ \\
2.5 & 40.54 & 51.05 & 0.13 & 0.02 & -3.28 & 38.76 & 0.017 & $0.27 \mathrm{E}+06$ \\
3.0 & 40.57 & 51.06 & 0.18 & 0.04 & -3.28 & 38.77 & 0.017 & $0.26 \mathrm{E}+06$ \\
3.5 & 40.75 & 51.03 & 0.41 & 0.28 & -3.30 & 38.78 & 0.016 & $0.26 \mathrm{E}+06$ \\
4.0 & 40.52 & 50.79 & 0.42 & 0.30 & -3.55 & 38.53 & 0.009 & $0.14 \mathrm{E}+06$ \\
4.5 & 40.39 & 50.71 & 0.42 & 0.27 & -3.63 & 38.43 & 0.008 & $0.12 \mathrm{E}+06$ \\
5.0 & 40.38 & 50.74 & 0.40 & 0.21 & -3.60 & 38.46 & 0.008 & $0.13 \mathrm{E}+06$ \\
5.1 & 40.36 & 50.61 & 0.47 & 0.33 & -3.73 & 38.36 & 0.006 & $0.95 \mathrm{E}+05$ \\
5.2 & 40.07 & 50.33 & 0.46 & 0.33 & -4.01 & 38.07 & 0.003 & $0.49 \mathrm{E}+05$ \\
5.3 & 39.99 & 50.27 & 0.42 & 0.30 & -4.06 & 38.00 & 0.003 & $0.45 \mathrm{E}+05$ \\
5.4 & 39.88 & 50.15 & 0.41 & 0.30 & -4.18 & 37.88 & 0.002 & $0.34 \mathrm{E}+05$ \\
\hline
\end{tabular}

Table 5.b. Emission line spectrum at solar metallicity (I)

\begin{tabular}{cccccccc}
\hline \hline Age & Ly $\alpha$ 1216 & HeII 1640 & MgII 2800 & [OII] 3727 & HeII 4686 & [OIII] 5007 & [OI] 6300 \\
\hline 1.5 & 23.05 & 0.00 & 0.05 & 3.22 & 0.00 & 0.67 & 0.11 \\
2.0 & 22.97 & 0.00 & 0.03 & 2.77 & 0.00 & 0.33 & 0.06 \\
2.5 & 22.94 & 0.00 & 0.03 & 2.48 & 0.00 & 0.22 & 0.05 \\
3.0 & 22.99 & 0.00 & 0.04 & 2.79 & 0.00 & 0.44 & 0.09 \\
3.5 & 24.01 & 0.93 & 0.11 & 6.04 & 0.10 & 2.24 & 0.49 \\
4.0 & 24.24 & 0.99 & 0.13 & 7.40 & 0.11 & 1.37 & 0.72 \\
4.5 & 24.22 & 0.53 & 0.16 & 7.39 & 0.06 & 1.00 & 0.69 \\
5.0 & 23.81 & 0.39 & 0.14 & 6.64 & 0.04 & 0.89 & 0.52 \\
5.1 & 24.55 & 0.94 & 0.17 & 8.57 & 0.10 & 0.89 & 0.95 \\
5.2 & 24.63 & 0.76 & 0.20 & 8.30 & 0.08 & 0.32 & 1.26 \\
5.3 & 24.41 & 0.64 & 0.19 & 7.49 & 0.07 & 0.23 & 1.26 \\
5.4 & 24.34 & 0.49 & 0.19 & 6.83 & 0.05 & 0.13 & 1.42 \\
\hline
\end{tabular}

Table 5.c. Emissicn line spectrum at solar metallicity (II)

\begin{tabular}{cccccccc}
\hline \hline Age & [NII] 6584 & [SII] 6716 & [SII] 6731 & [SIII] 9532 & [CII] 158 $\mu \mathrm{m}$ & EW(H $\beta)$ & $T_{e}(K)$ \\
\hline 1.5 & 1.40 & 0.74 & 0.51 & 0.84 & 1.72 & 537.50 & 6960 \\
2.0 & 1.34 & 0.70 & 0.49 & 0.69 & 1.92 & 411.24 & 6730 \\
2.5 & 1.28 & 0.69 & 0.47 & 0.62 & 2.02 & 357.60 & 6600 \\
3.0 & 1.33 & 0.73 & 0.51 & 0.70 & 1.90 & 333.62 & 6760 \\
3.5 & 2.27 & 1.62 & 1.12 & 1.12 & 2.99 & 313.41 & 7880 \\
4.0 & 2.83 & 2.22 & 1.53 & 0.93 & 3.95 & 98.70 & 7930 \\
4.5 & 2.84 & 2.16 & 1.49 & 0.86 & 3.60 & 189.49 & 7950 \\
5.0 & 2.57 & 1.82 & 1.26 & 0.89 & 2.96 & 254.36 & 7900 \\
5.1 & 3.26 & 2.70 & 1.86 & 0.83 & 4.68 & 210.37 & 8020 \\
5.2 & 3.40 & 3.26 & 2.25 & 0.57 & 5.77 & 108.20 & 7910 \\
5.3 & 3.21 & 3.21 & 2.21 & 0.49 & 5.88 & 88.33 & 7780 \\
5.4 & 3.12 & 3.40 & 2.34 & 0.41 & 6.96 & 66.44 & 7550 \\
\hline
\end{tabular}

Table 5.d. Emission line spectrum at twice solar metallicity (I)

\begin{tabular}{cccccccc}
\hline \hline Age & Ly $\alpha$ 1216 & HeII 1640 & MgII 2800 & [OII] 3727 & HeII 4686 & [OIII] 5007 & [OI] 6300 \\
\hline 1.5 & 22.64 & 0.00 & 0.01 & 1.28 & 0.00 & 0.19 & 0.08 \\
2.0 & 22.64 & 0.00 & 0.01 & 0.93 & 0.00 & 0.09 & 0.04 \\
2.5 & 22.63 & 0.00 & 0.01 & 0.77 & 0.00 & 0.06 & 0.03 \\
3.0 & 22.61 & 0.00 & 0.01 & 0.99 & 0.00 & 0.11 & 0.06 \\
3.5 & 22.88 & 0.89 & 0.05 & 3.72 & 0.10 & 0.92 & 0.45 \\
4.0 & 22.95 & 0.92 & 0.06 & 4.48 & 0.10 & 0.70 & 0.63 \\
4.5 & 22.94 & 0.49 & 0.07 & 4.44 & 0.05 & 0.52 & 0.61 \\
5.0 & 22.88 & 0.36 & 0.06 & 3.67 & 0.04 & 0.43 & 0.44 \\
5.1 & 23.01 & 0.89 & 0.08 & 5.54 & 0.10 & 0.55 & 0.85 \\
5.2 & 22.97 & 0.71 & 0.09 & 5.04 & 0.08 & 0.20 & 1.06 \\
5.3 & 22.92 & 0.60 & 0.08 & 4.27 & 0.07 & 0.13 & 0.99 \\
5.4 & 22.88 & 0.44 & 0.07 & 3.75 & 0.05 & 0.08 & 103 \\
\hline
\end{tabular}

Table 5.e. Emission line spectrum at twice solar metallicity (II)

\begin{tabular}{cccccccc}
\hline \hline Age & [NII] 6584 & [SII] 6716 & [SII] 6731 & [SIII] 9532 & [CII] 158 $\mu \mathrm{m}$ & $\mathrm{EW}(\mathrm{H} \beta)$ & $T_{e}(K)$ \\
\hline 1.5 & 0.99 & 0.57 & 0.39 & 0.63 & 3.00 & 563.54 & 5190 \\
2.0 & 0.87 & 0.51 & 0.35 & 0.52 & 3.29 & 426.10 & 5060 \\
2.5 & 0.80 & 0.47 & 0.33 & 0.46 & 3.45 & 368.93 & 4930 \\
3.0 & 0.86 & 0.53 & 0.36 & 0.50 & 3.24 & 344.45 & 5030 \\
3.5 & 2.14 & 1.57 & 1.08 & 1.01 & 4.78 & 314.03 & 6170 \\
4.0 & 2.71 & 2.18 & 1.50 & 0.93 & 6.30 & 96.68 & 6370 \\
4.5 & 2.70 & 2.14 & 1.47 & 0.88 & 5.82 & 188.24 & 6410 \\
5.0 & 2.32 & 1.74 & 1.20 & 0.88 & 5.08 & 256.56 & 6240 \\
5.1 & 3.29 & 2.77 & 1.91 & 0.91 & 7.51 & 205.53 & 6550 \\
5.2 & 3.31 & 3.23 & 2.22 & 0.64 & 9.44 & 104.52 & 6430 \\
5.3 & 2.98 & 3.04 & 2.09 & 0.54 & 9.71 & 86.18 & 6250 \\
5.4 & 2.79 & 3.07 & 2.11 & 0.44 & 10.86 & 64.75 & 6080 \\
\hline
\end{tabular}


TABLE 6

$M_{T}=0.4 \times 10^{5} M_{\odot}$, IMF PARAMETERS: $\alpha=2.35, m_{\mathrm{low}}=0.85 M_{\odot}, m_{\mathrm{up}}=120 M_{\odot}$

Table 6.a. Parameters independent of metallicity

\begin{tabular}{ccccccccc}
\hline \hline Age & $\log L_{L y_{\alpha}}$ & $\log Q H$ & $\mathrm{QHe}_{\mathrm{QH}}$ & $\mathrm{QO} / \mathrm{QH}$ & $\log u$ & $\log I_{H_{\beta}}$ & $\epsilon$ & $M_{H I I} / \mathrm{M}_{\odot}$ \\
\hline 1.5 & 40.98 & 51.45 & 0.24 & 0.06 & -2.88 & 39.16 & 0.043 & $0.69 \mathrm{E}+06$ \\
2.0 & 40.91 & 51.41 & 0.16 & 0.03 & -2.93 & 39.11 & 0.039 & $0.60 \mathrm{E}+06$ \\
2.5 & 40.88 & 51.40 & 0.13 & 0.02 & -2.94 & 39.10 & 0.038 & $0.58 \mathrm{E}+06$ \\
3.0 & 40.92 & 51.41 & 0.19 & 0.05 & -2.93 & 39.12 & 0.039 & $0.60 \mathrm{E}+06$ \\
3.5 & 41.12 & 51.40 & 0.43 & 0.29 & -2.94 & 39.13 & 0.038 & $0.59 \mathrm{E}+06$ \\
4.0 & 40.90 & 51.14 & 0.46 & 0.33 & -3.20 & 38.89 & 0.021 & $0.32 \mathrm{E}+06$ \\
4.5 & 40.69 & 51.01 & 0.42 & 0.27 & -3.32 & 38.74 & 0.016 & $0.25 \mathrm{E}+06$ \\
5.0 & 40.70 & 51.06 & 0.40 & 0.21 & -3.28 & 38.77 & 0.017 & $0.27 \mathrm{E}+06$ \\
5.1 & 40.67 & 50.92 & 0.48 & 0.34 & -3.41 & 38.67 & 0.013 & $0.20 \mathrm{E}+06$ \\
5.2 & 40.40 & 50.66 & 0.47 & 0.34 & -3.68 & 38.40 & 0.007 & $0.11 \mathrm{E}+06$ \\
5.3 & 40.32 & 50.60 & 0.43 & 0.31 & -3.74 & 38.34 & 0.006 & $0.93 \mathrm{E}+05$ \\
5.4 & 40.21 & 50.48 & 0.42 & 0.31 & -3.86 & 38.22 & 0.005 & $0.70 \mathrm{E}+05$ \\
\hline
\end{tabular}

Table 6.b. Emission line spectrum at solar metallicity (I)

\begin{tabular}{cccccccc}
\hline \hline Age & Ly $\alpha 1216$ & HeII 1640 & MgII 2800 & [OII] 3727 & HeII 4686 & [OIII] 5007 & [OI] 6300 \\
\hline 1.5 & 22.95 & 0.00 & 0.03 & 2.19 & 0.00 & 0.97 & 0.06 \\
2.0 & 22.91 & 0.00 & 0.02 & 2.06 & 0.00 & 0.57 & 0.03 \\
2.5 & 22.89 & 0.00 & 0.02 & 1.94 & 0.00 & 0.38 & 0.03 \\
3.0 & 22.92 & 0.00 & 0.03 & 1.97 & 0.00 & 0.70 & 0.05 \\
3.5 & 23.89 & 1.14 & 0.09 & 4.36 & 0.13 & 3.91 & 0.31 \\
4.0 & 24.22 & 1.32 & 0.11 & 6.17 & 0.14 & 3.24 & 0.51 \\
4.5 & 24.10 & 0.63 & 0.13 & 6.22 & 0.07 & 2.03 & 0.46 \\
5.0 & 23.73 & 0.45 & 0.11 & 5.51 & 0.05 & 1.81 & 0.34 \\
5.1 & 24.39 & 1.19 & 0.13 & 7.51 & 0.13 & 2.22 & 0.65 \\
5.2 & 24.59 & 1.04 & 0.16 & 8.46 & 0.11 & 1.07 & 0.89 \\
5.3 & 24.40 & 0.90 & 0.16 & 7.93 & 0.10 & 0.79 & 0.89 \\
5.4 & 24.60 & 0.71 & 0.17 & 7.87 & 0.08 & 0.49 & 1.11 \\
\hline
\end{tabular}

Table 6.c. Emission line spectrum at solar metallicity (II)

\begin{tabular}{cccccccc}
\hline \hline Age & {$[\mathrm{NII}] 6584$} & [SII] 6716 & [SII] 6731 & [SIII] 9532 & {$[\mathrm{CII}] 158 \mu \mathrm{m}$} & $\mathrm{EW}\left(\mathrm{H}_{\beta}\right)$ & $T_{e}\left(K^{\prime}\right)$ \\
\hline 1.5 & 0.90 & 0.43 & 0.30 & 0.85 & 1.17 & 558.36 \\
2.0 & 0.96 & 0.43 & 0.29 & 0.77 & 1.44 & 420.78 & 6440 \\
2.5 & 0.99 & 0.43 & 0.30 & 0.72 & 1.64 & 357.43 & 6390 \\
3.0 & 0.91 & 0.44 & 0.30 & 0.75 & 1.37 & 338.43 & 6410 \\
3.5 & 1.52 & 1.05 & 0.72 & 1.40 & 1.91 & 325.85 & 7930 \\
4.0 & 2.22 & 1.61 & 1.11 & 1.31 & 2.84 & 111.08 & 8090 \\
4.5 & 2.28 & 1.58 & 1.09 & 1.09 & 2.60 & 186.76 & 7920 \\
5.0 & 2.03 & 1.28 & 0.89 & 1.10 & 2.06 & 258.01 & 7810 \\
5.1 & 2.73 & 2.03 & 1.40 & 1.13 & 3.40 & 213.17 & 8130 \\
5.2 & 3.20 & 2.62 & 1.81 & 0.85 & 4.32 & 113.85 & 8080 \\
5.3 & 3.10 & 2.61 & 1.80 & 0.75 & 4.44 & 94.12 & 7970 \\
5.4 & 3.20 & 2.94 & 2.03 & 0.64 & 5.44 & 70.72 & 7810 \\
\hline
\end{tabular}

Table 6.d. Emission line spectrum at twice solar metallicity (I)

\begin{tabular}{cccccccc}
\hline \hline Age & Ly $\alpha 1216$ & HeII 1640 & MgII 2800 & [OII] 3727 & HeII 4686 & [OIII] 5007 & [OI] 6300 \\
\hline 1.5 & 22.39 & 0.00 & 0.01 & 0.91 & 0.00 & 0.20 & 0.05 \\
2.0 & 22.46 & 0.00 & 0.01 & 0.68 & 0.00 & 0.10 & 0.02 \\
2.5 & 22.48 & 0.00 & 0.00 & 0.56 & 0.00 & 0.06 & 0.02 \\
3.0 & 22.39 & 0.00 & 0.01 & 0.71 & 0.00 & 0.12 & 0.03 \\
3.5 & 22.80 & 1.10 & 0.05 & 2.91 & 0.12 & 1.22 & 0.30 \\
4.0 & 22.94 & 1.26 & 0.06 & 4.04 & 0.14 & 1.35 & 0.48 \\
4.5 & 22.88 & 0.59 & 0.06 & 3.85 & 0.07 & 0.80 & 0.44 \\
5.0 & 22.80 & 0.42 & 0.05 & 3.12 & 0.05 & 0.66 & 0.31 \\
5.1 & 23.00 & 1.13 & 0.07 & 4.89 & 0.12 & 1.11 & 0.60 \\
5.2 & 23.02 & 0.98 & 0.08 & 5.49 & 0.11 & 0.62 & 0.81 \\
5.3 & 22.98 & 0.84 & 0.08 & 4.90 & 0.09 & 0.45 & 0.79 \\
5.4 & 22.97 & 0.66 & 0.08 & 4.78 & 0.07 & 0.30 & 0.92 \\
\hline
\end{tabular}

Table 6.e. Emission line spectrum at twice solar metallicity (II)

\begin{tabular}{cccccccc}
\hline \hline Age & {$[\mathrm{NII}] 6584$} & [SII] 6716 & [SII] 6731 & [SIII] 9532 & [CII] $158 \mu \mathrm{m}$ & $\mathrm{EW}(\mathrm{H} \beta)$ & $T_{e}\left(\mathrm{~K}^{\prime}\right)$ \\
\hline 1.5 & 0.62 & 0.33 & 0.22 & 0.48 & 1.96 & 593.74 \\
2.0 & 0.58 & 0.29 & 0.20 & 0.46 & 2.41 & 442.16 & 4580 \\
2.5 & 0.56 & 0.28 & 0.19 & 0.42 & 2.72 & 373.11 & 4560 \\
3.0 & 0.56 & 0.30 & 0.21 & 0.41 & 2.26 & 349.80 & 4480 \\
3.5 & 1.47 & 1.00 & 0.69 & 1.03 & 3.17 & 332.44 & 5840 \\
4.0 & 2.18 & 1.59 & 1.10 & 1.17 & 4.66 & 108.26 & 6280 \\
4.5 & 2.14 & 1.55 & 1.07 & 0.96 & 4.23 & 185.06 & 6220 \\
5.0 & 1.79 & 1.22 & 0.84 & 0.93 & 3.53 & 260.61 & 6010 \\
5.1 & 2.72 & 2.06 & 1.42 & 1.13 & 5.57 & 210.49 & 6490 \\
5.2 & 3.22 & 2.70 & 1.86 & 0.92 & 7.08 & 110.34 & 6580 \\
5.3 & 3.02 & 2.63 & 1.81 & 0.80 & 7.29 & 91.52 & 6460 \\
5.4 & 3.08 & 2.87 & 1.97 & 0.69 & 8.49 & 68.37 & 6380 \\
\hline
\end{tabular}


TABLE 7

$$
M_{T}=0.6 \times 10^{5} M_{\odot} \text {, IMF PARAMETERS: } \alpha=2.35, m_{\mathrm{low}}=0.85 M_{\odot}, m_{\mathrm{up}}=120 M_{\odot}
$$

Table 7.a. Parameters independent of metallicity

\begin{tabular}{ccccccccc}
\hline \hline Age & $\log L_{L y \alpha}$ & $\log Q H$ & $\mathrm{QHe} / \mathrm{QH}$ & $\mathrm{QO} / \mathrm{QH}$ & $\log u$ & $\log I_{H_{\beta}}$ & $\epsilon$ & $M_{H I I} / \mathrm{M}_{\odot}$ \\
\hline 1.5 & 41.17 & 51.65 & 0.24 & 0.06 & -2.69 & 39.35 & 0.067 & $0.10 \mathrm{E}+07$ \\
2.0 & 41.10 & 51.60 & 0.16 & 0.03 & -2.73 & 39.30 & 0.061 & $0.98 \mathrm{E}+06$ \\
2.5 & 41.08 & 51.59 & 0.12 & 0.02 & -2.74 & 39.29 & 0.060 & $0.95 \mathrm{E}+06$ \\
3.0 & 41.12 & 51.61 & 0.19 & 0.05 & -2.73 & 39.32 & 0.062 & $0.94 \mathrm{E}+06$ \\
3.5 & 41.33 & 51.60 & 0.45 & 0.31 & -2.74 & 39.33 & 0.060 & $0.92 \mathrm{E}+06$ \\
4.0 & 41.08 & 51.33 & 0.46 & 0.33 & -3.01 & 39.07 & 0.032 & $0.50 \mathrm{E}+06$ \\
4.5 & 40.87 & 51.19 & 0.42 & 0.27 & -3.15 & 38.92 & 0.024 & $0.36 \mathrm{E}+06$ \\
5.0 & 40.88 & 51.24 & 0.40 & 0.21 & -3.10 & 38.95 & 0.026 & $0.40 \mathrm{E}+06$ \\
5.1 & 40.86 & 51.11 & 0.48 & 0.34 & -3.23 & 38.85 & 0.019 & $0.30 \mathrm{E}+06$ \\
5.2 & 40.58 & 50.84 & 0.47 & 0.34 & -3.50 & 38.58 & 0.010 & $0.16 \mathrm{E}+06$ \\
5.3 & 40.51 & 50.78 & 0.43 & 0.31 & -3.56 & 38.52 & 0.009 & $0.14 \mathrm{E}+06$ \\
5.4 & 40.40 & 50.66 & 0.42 & 0.31 & -3.68 & 38.40 & 0.007 & $0.11 \mathrm{E}+06$ \\
\hline
\end{tabular}

Table 7.b. Emission line spectrum at solar metallicity (I)

\begin{tabular}{cccccccc}
\hline \hline Age & Ly $\alpha 1216$ & HeII 1640 & MgII 2800 & [OII] 3727 & HeII 4686 & [OIII] 5007 & [OI] 6300 \\
\hline 1.5 & 22.90 & 0.00 & 0.03 & 1.71 & 0.00 & 1.08 & 0.05 \\
2.0 & 22.87 & 0.00 & 0.02 & 1.63 & 0.00 & 0.63 & 0.02 \\
2.5 & 22.86 & 0.00 & 0.01 & 1.63 & 0.00 & 0.43 & 0.02 \\
3.0 & 22.86 & 0.00 & 0.02 & 1.54 & 0.00 & 0.79 & 0.04 \\
3.5 & 23.86 & 1.24 & 0.08 & 3.64 & 0.14 & 5.03 & 0.23 \\
4.0 & 24.10 & 1.41 & 0.10 & 5.10 & 0.15 & 4.14 & 0.39 \\
4.5 & 23.96 & 0.67 & 0.11 & 5.24 & 0.07 & 2.66 & 0.35 \\
5.0 & 23.66 & 0.48 & 0.10 & 4.69 & 0.05 & 2.35 & 0.26 \\
5.1 & 24.28 & 1.31 & 0.12 & 6.55 & 0.14 & 3.16 & 0.52 \\
5.2 & 24.49 & 1.19 & 0.14 & 7.95 & 0.13 & 1.81 & 0.71 \\
5.3 & 24.33 & 1.05 & 0.14 & 7.57 & 0.11 & 1.41 & 0.72 \\
5.4 & 24.57 & 0.85 & 0.16 & 7.90 & 0.09 & 0.96 & 0.93 \\
\hline
\end{tabular}

Table 7.c. Emission line spectrum at solar metallicity (II)

\begin{tabular}{cccccccc}
\hline Age & {$[\mathrm{NII}] 6584$} & {$[\mathrm{SII}] 6716$} & [SII] 6731 & [SIII] 9532 & [CII] 158 $\mu \mathrm{m}$ & $\mathrm{EW}\left(\mathrm{H}_{\beta}\right)$ & $T_{e}(K)$ \\
\hline 1.5 & 0.68 & 0.31 & 0.22 & 0.81 & 0.91 & 569.59 & 6310 \\
2.0 & 0.75 & 0.30 & 0.21 & 0.75 & 1.21 & 423.00 & 6190 \\
2.5 & 0.82 & 0.32 & 0.22 & 0.73 & 1.45 & 358.64 & 6180 \\
3.0 & 0.68 & 0.31 & 0.22 & 0.72 & 1.07 & 349.66 & 6130 \\
3.5 & 1.19 & 0.80 & 0.55 & 1.56 & 1.42 & 334.20 & 8040 \\
4.0 & 1.78 & 1.27 & 0.87 & 1.45 & 2.22 & 111.46 & 8120 \\
4.5 & 1.88 & 1.25 & 0.87 & 1.19 & 2.09 & 188.13 & 7880 \\
5.0 & 1.66 & 1.01 & 0.70 & 1.18 & 1.63 & 257.95 & 7730 \\
5.1 & 2.32 & 1.67 & 1.15 & 1.29 & 2.80 & 210.63 & 8160 \\
5.2 & 2.91 & 2.23 & 1.54 & 1.02 & 3.62 & 113.66 & 8130 \\
5.3 & 2.86 & 2.25 & 1.55 & 0.91 & 3.78 & 94.00 & 8010 \\
5.4 & 3.08 & 2.62 & 1.81 & 0.80 & 4.71 & 71.53 & 7900 \\
\hline
\end{tabular}

Table 7.d. Emission line spectrum at twice solar metallicity (I)

\begin{tabular}{cccccccc}
\hline \hline Age & Ly $\alpha 1216$ & HeII 1640 & MgII 2800 & [OII] 3727 & HeII 4686 & [OIII] 5007 & [OI] 6300 \\
\hline 1.5 & 22.22 & 0.00 & 0.01 & 0.71 & 0.00 & 0.19 & 0.04 \\
2.0 & 22.32 & 0.00 & 0.00 & 0.55 & 0.00 & 0.09 & 0.02 \\
2.5 & 22.37 & 0.00 & 0.00 & 0.47 & 0.00 & 0.05 & 0.01 \\
3.0 & 22.20 & 0.00 & 0.01 & 0.57 & 0.00 & 0.12 & 0.03 \\
3.5 & 22.64 & 1.20 & 0.04 & 2.65 & 0.13 & 1.54 & 0.24 \\
4.0 & 22.90 & 1.36 & 0.05 & 3.49 & 0.15 & 1.54 & 0.38 \\
4.5 & 22.82 & 0.64 & 0.06 & 3.47 & 0.07 & 0.93 & 0.36 \\
5.0 & 22.72 & 0.45 & 0.05 & 2.75 & 0.05 & 0.74 & 0.25 \\
5.1 & 22.97 & 1.25 & 0.06 & 4.33 & 0.14 & 1.37 & 0.49 \\
5.2 & 23.01 & 1.13 & 0.07 & 5.10 & 0.12 & 0.94 & 0.66 \\
5.3 & 22.98 & 0.99 & 0.07 & 4.70 & 0.11 & 0.72 & 0.65 \\
5.4 & 22.99 & 0.79 & 0.08 & 4.90 & 0.09 & 0.52 & 0.80 \\
\hline
\end{tabular}

Table 7.e. Emission line spectrum at twice solar metallicity (II)

\begin{tabular}{cccccccc}
\hline \hline Age & {$[\mathrm{NII}] 6584$} & [SII] 6716 & [SII] 6731 & [SIII] 9532 & [CII] 158 $\mu \mathrm{m}$ & $\mathrm{EW}\left(\mathrm{H}_{\beta}\right)$ & $T_{e}(K)$ \\
\hline 1.5 & 0.46 & 0.23 & 0.16 & 0.39 & 1.47 & 610.16 & 4230 \\
2.0 & 0.45 & 0.20 & 0.14 & 0.39 & 1.97 & 446.02 & 4250 \\
2.5 & 0.45 & 0.20 & 0.14 & 0.38 & 2.35 & 376.30 & 4300 \\
3.0 & 0.42 & 0.21 & 0.15 & 0.33 & 1.71 & 364.18 & 4100 \\
3.5 & 1.22 & 0.79 & 0.54 & 1.07 & 2.44 & 341.07 & 5750 \\
4.0 & 1.78 & 1.25 & 0.86 & 1.19 & 3.70 & 108.78 & 6120 \\
4.5 & 1.81 & 1.25 & 0.86 & 0.95 & 3.43 & 183.19 & 6030 \\
5.0 & 1.48 & 0.96 & 0.66 & 0.89 & 2.79 & 260.14 & 5790 \\
5.1 & 2.30 & 1.67 & 1.15 & 1.19 & 4.61 & 208.70 & 6370 \\
5.2 & 2.89 & 2.27 & 1.56 & 1.04 & 5.88 & 111.18 & 6550 \\
5.3 & 2.78 & 2.25 & 1.55 & 0.92 & 6.10 & 91.84 & 6450 \\
5.4 & 2.99 & 2.58 & 1.77 & 0.83 & 7.38 & 69.51 & 6420 \\
\hline
\end{tabular}


TABLE 8

$M_{T}=0.4 \times 10^{5} M_{\odot}$, IMF PARAMETERS: $\alpha=2.35, m_{\mathrm{low}}=0.85 M_{\odot}, m_{\mathrm{up}}=60 M_{\odot}$

Table 8.a. Parameters independent of metallicity

\begin{tabular}{ccccccccc}
\hline Age & $\log L_{L y \alpha}$ & $\log Q H$ & $\mathrm{QHe} / \mathrm{QH}$ & $\mathrm{QO} / \mathrm{QH}$ & $\log u$ & $\log I_{H \beta}$ & $\epsilon$ & $M_{H I I} / \mathrm{M}_{\odot}$ \\
\hline 1.5 & 40.67 & 51.15 & 0.21 & 0.05 & -3.18 & 38.86 & 0.022 & $0.34 \mathrm{E}+06$ \\
2.0 & 40.62 & 51.12 & 0.16 & 0.03 & -3.21 & 38.83 & 0.020 & $0.33 \mathrm{E}+06$ \\
2.5 & 40.63 & 51.14 & 0.15 & 0.03 & -3.20 & 38.84 & 0.021 & $0.32 \mathrm{E}+06$ \\
3.0 & 40.60 & 51.12 & 0.12 & 0.02 & -3.22 & 38.82 & 0.020 & $0.30 \mathrm{E}+06$ \\
3.5 & 40.43 & 50.97 & 0.06 & 0.01 & -3.36 & 38.67 & 0.014 & $0.23 \mathrm{E}+06$ \\
4.0 & 40.42 & 50.89 & 0.15 & 0.08 & -3.44 & 38.60 & 0.012 & $0.19 \mathrm{E}+06$ \\
4.5 & 40.70 & 51.02 & 0.41 & 0.26 & -3.32 & 38.74 & 0.016 & $0.24 \mathrm{E}+06$ \\
5.0 & 40.73 & 51.09 & 0.40 & 0.21 & -3.25 & 38.80 & 0.019 & $0.29 \mathrm{E}+06$ \\
5.1 & 40.71 & 50.95 & 0.49 & 0.34 & -3.39 & 38.70 & 0.014 & $0.20 \mathrm{E}+06$ \\
5.2 & 40.43 & 50.68 & 0.47 & 0.34 & -3.66 & 38.43 & 0.007 & $0.11 \mathrm{E}+06$ \\
5.3 & 40.35 & 50.62 & 0.43 & 0.31 & -3.71 & 38.37 & 0.006 & $0.10 \mathrm{E}+06$ \\
5.4 & 40.24 & 50.50 & 0.42 & 0.31 & -3.83 & 38.24 & 0.005 & $0.77 \mathrm{E}+05$ \\
\hline
\end{tabular}

Table 8.b. Emission line spectrum at solar metallicity (I)

\begin{tabular}{cccccccc}
\hline \hline Age & Ly $\alpha$ 1216 & HeII 1640 & MgII 2800 & [OII] 3727 & HeII 4686 & [OIII] 5007 & [OI] 6300 \\
\hline 1.5 & 23.00 & 0.00 & 0.04 & 2.90 & 0.00 & 0.63 & 0.08 \\
2.0 & 22.96 & 0.00 & 0.03 & 2.64 & 0.00 & 0.40 & 0.06 \\
2.5 & 22.94 & 0.00 & 0.03 & 2.55 & 0.00 & 0.36 & 0.05 \\
3.0 & 22.92 & 0.00 & 0.02 & 2.34 & 0.00 & 0.23 & 0.04 \\
3.5 & 22.90 & 0.00 & 0.02 & 1.87 & 0.00 & 0.04 & 0.05 \\
4.0 & 22.99 & 0.09 & 0.04 & 2.90 & 0.01 & 0.38 & 0.13 \\
4.5 & 24.06 & 0.62 & 0.13 & 6.08 & 0.07 & 2.01 & 0.45 \\
5.0 & 23.75 & 0.47 & 0.11 & 5.46 & 0.05 & 1.93 & 0.33 \\
5.1 & 24.41 & 1.22 & 0.13 & 7.47 & 0.13 & 2.40 & 0.63 \\
5.2 & 24.62 & 1.08 & 0.16 & 8.51 & 0.12 & 1.18 & 0.87 \\
5.3 & 24.44 & 0.94 & 0.16 & 8.00 & 0.10 & 0.88 & 0.87 \\
5.4 & 24.64 & 0.74 & 0.17 & 7.99 & 0.08 & 0.56 & 1.10 \\
\hline
\end{tabular}

Table 8.c. Emission line spectrum at solar metallicity (II)

\begin{tabular}{cccccccc}
\hline \hline Age & {$[\mathrm{NII}] 6584$} & {$[\mathrm{SII}] 6716$} & {$[\mathrm{SII}] 6731$} & {$[\mathrm{SIII}] 9532$} & {$[\mathrm{CII}] 158 \mu \mathrm{m}$} & $\mathrm{EW}(\mathrm{H} \beta)$ & $T_{e}\left(\mathrm{~K}^{r}\right)$ \\
\hline 1.5 & 1.29 & 0.66 & 0.46 & 0.81 & 1.68 & 421.59 & 6840 \\
2.0 & 1.27 & 0.65 & 0.45 & 0.72 & 1.83 & 352.38 & 6690 \\
2.5 & 1.25 & 0.63 & 0.43 & 0.71 & 1.85 & 339.68 & 6660 \\
3.0 & 1.23 & 0.63 & 0.44 & 0.63 & 1.99 & 294.79 & 6570 \\
3.5 & 1.14 & 0.70 & 0.48 & 0.40 & 2.36 & 171.54 & 6300 \\
4.0 & 1.46 & 0.98 & 0.68 & 0.52 & 2.39 & 59.14 & 6820 \\
4.5 & 2.24 & 1.55 & 1.07 & 1.08 & 2.59 & 179.51 & 7880 \\
5.0 & 1.99 & 1.25 & 0.86 & 1.13 & 1.99 & 261.46 & 7820 \\
5.1 & 2.69 & 1.99 & 1.37 & 1.17 & 3.34 & 215.70 & 8140 \\
5.2 & 3.19 & 2.58 & 1.78 & 0.88 & 4.19 & 114.92 & 8120 \\
5.3 & 3.10 & 2.58 & 1.78 & 0.78 & 4.33 & 94.73 & 8000 \\
5.4 & 3.22 & 2.93 & 2.02 & 0.66 & 5.40 & 71.39 & 7830 \\
\hline
\end{tabular}

Table 8.d. Emission line spectrum at twice solar metallicity (I)

\begin{tabular}{cccccccc}
\hline Age & Ly $\alpha 1216$ & HeII 1640 & MgII 2800 & [OII] 3727 & HeII 4686 & [OIII] 5007 & [OI] 6300 \\
\hline 1.5 & 22.60 & 0.00 & 0.01 & 1.11 & 0.00 & 0.16 & 0.06 \\
2.0 & 22.61 & 0.00 & 0.01 & 0.87 & 0.00 & 0.10 & 0.04 \\
2.5 & 22.61 & 0.00 & 0.01 & 0.83 & 0.00 & 0.08 & 0.03 \\
3.0 & 22.60 & 0.00 & 0.00 & 0.67 & 0.00 & 0.05 & 0.03 \\
3.5 & 22.56 & 0.00 & 0.00 & 0.47 & 0.00 & 0.01 & 0.03 \\
4.0 & 22.63 & 0.08 & 0.01 & 1.00 & 0.01 & 0.10 & 0.08 \\
4.5 & 22.87 & 0.59 & 0.06 & 3.77 & 0.06 & 0.78 & 0.43 \\
5.0 & 22.79 & 0.44 & 0.05 & 3.12 & 0.05 & 0.69 & 0.31 \\
5.1 & 23.00 & 1.17 & 0.07 & 4.90 & 0.13 & 1.19 & 0.59 \\
5.2 & 23.03 & 1.02 & 0.08 & 5.54 & 0.11 & 0.67 & 0.80 \\
5.3 & 22.99 & 0.88 & 0.08 & 4.99 & 0.10 & 0.50 & 0.78 \\
5.4 & 22.98 & 0.69 & 0.08 & 4.95 & 0.08 & 0.33 & 0.92 \\
\hline
\end{tabular}

Table 8.e. Emission line spectrum at twice solar metallicity (II)

\begin{tabular}{cccccccc}
\hline \hline Age & {$[\mathrm{NII}] 6584$} & {$[\mathrm{SII}] 6716$} & {$[\mathrm{SII}] 6731$} & {$[\mathrm{SIII}] 9532$} & [CII] 158 $\mu \mathrm{m}$ & $\mathrm{EW}(\mathrm{H} \beta)$ & $T_{e}\left(K^{\prime}\right)$ \\
\hline 1.5 & 0.88 & 0.50 & 0.34 & 0.57 & 2.89 & 437.27 & 5060 \\
2.0 & 0.81 & 0.46 & 0.32 & 0.51 & 3.13 & 363.43 & 4970 \\
2.5 & 0.79 & 0.44 & 0.30 & 0.50 & 3.14 & 351.38 & 4950 \\
3.0 & 0.72 & 0.42 & 0.29 & 0.43 & 3.36 & 302.13 & 4840 \\
3.5 & 0.62 & 0.42 & 0.29 & 0.28 & 3.92 & 175.75 & 4710 \\
4.0 & 0.92 & 0.68 & 0.47 & 0.37 & 4.00 & 59.34 & 5090 \\
4.5 & 2.10 & 1.52 & 1.05 & 0.95 & 4.19 & 176.91 & 6180 \\
5.0 & 1.77 & 1.19 & 0.82 & 0.94 & 3.42 & 263.23 & 6000 \\
5.1 & 2.70 & 2.03 & 1.40 & 1.16 & 5.48 & 213.10 & 6480 \\
5.2 & 3.22 & 2.66 & 1.83 & 0.95 & 6.89 & 111.64 & 6600 \\
5.3 & 3.04 & 2.61 & 1.79 & 0.83 & 7.16 & 92.13 & 6480 \\
5.4 & 3.13 & 2.88 & 1.98 & 0.72 & 8.44 & 69.35 & 6390 \\
\hline
\end{tabular}


TABLE 9

$M_{T}=0.6 \times 10^{5} M_{\odot}$, IMF PARAMETERS: $\alpha=2.35, m_{\mathrm{low}}=0.85 M_{\odot}, m_{\mathrm{up}}=60 M_{\odot}$

Table 9.a. Parameters independent of metallicity

\begin{tabular}{ccccccccc}
\hline \hline Age & $\log L_{L y_{\alpha}}$ & $\log Q H$ & $\mathrm{QHe} / \mathrm{QH}$ & $\mathrm{QO} / \mathrm{QH}$ & $\log u$ & $\log I_{H}$ & $\epsilon$ & $M_{H I I} / \mathrm{M}_{\odot}$ \\
\hline 1.5 & 40.87 & 51.35 & 0.21 & 0.05 & -2.98 & 39.06 & 0.034 & $0.55 \mathrm{E}+06$ \\
2.0 & 40.82 & 51.32 & 0.16 & 0.03 & -3.02 & 39.02 & 0.032 & $0.48 \mathrm{E}+06$ \\
2.5 & 40.83 & 51.33 & 0.15 & 0.03 & -3.00 & 39.04 & 0.033 & $0.52 \mathrm{E}+06$ \\
3.0 & 40.80 & 51.32 & 0.12 & 0.02 & -3.02 & 39.02 & 0.032 & $0.49 \mathrm{E}+06$ \\
3.5 & 40.62 & 51.16 & 0.06 & 0.01 & -3.18 & 38.86 & 0.022 & $0.34 \mathrm{E}+06$ \\
4.0 & 40.64 & 51.10 & 0.18 & 0.09 & -3.23 & 38.81 & 0.019 & $0.31 \mathrm{E}+06$ \\
4.5 & 40.91 & 51.22 & 0.42 & 0.27 & -3.11 & 38.95 & 0.025 & $0.40 \mathrm{E}+06$ \\
5.0 & 40.92 & 51.27 & 0.40 & 0.21 & -3.06 & 39.00 & 0.029 & $0.45 \mathrm{E}+06$ \\
5.1 & 40.89 & 51.14 & 0.49 & 0.34 & -3.20 & 38.88 & 0.021 & $0.32 \mathrm{E}+06$ \\
5.2 & 40.62 & 50.88 & 0.48 & 0.35 & -3.46 & 38.62 & 0.011 & $0.18 \mathrm{E}+06$ \\
5.3 & 40.54 & 50.82 & 0.44 & 0.32 & -3.52 & 38.56 & 0.010 & $0.16 \mathrm{E}+06$ \\
5.4 & 40.43 & 50.69 & 0.43 & 0.32 & -3.64 & 38.44 & 0.008 & $0.12 \mathrm{E}+06$ \\
\hline
\end{tabular}

Table 9.b. Emission line spectrum at solar metallicity (I)

\begin{tabular}{cccccccc}
\hline \hline Age & Ly $\alpha$ 1216 & HeII 1640 & MgII 2800 & [OII] 3727 & HeII 4686 & [OIII] 5007 & [OI] 6300 \\
\hline 1.5 & 22.95 & 0.00 & 0.03 & 2.36 & 0.00 & 0.80 & 0.06 \\
2.0 & 22.93 & 0.00 & 0.03 & 2.22 & 0.00 & 0.53 & 0.04 \\
2.5 & 22.92 & 0.00 & 0.02 & 2.19 & 0.00 & 0.49 & 0.04 \\
3.0 & 22.90 & 0.00 & 0.02 & 2.02 & 0.00 & 0.31 & 0.03 \\
3.5 & 22.88 & 0.00 & 0.02 & 1.82 & 0.00 & 0.06 & 0.04 \\
4.0 & 23.04 & 0.13 & 0.04 & 2.70 & 0.01 & 0.68 & 0.13 \\
4.5 & 23.98 & 0.69 & 0.11 & 5.15 & 0.08 & 2.82 & 0.34 \\
5.0 & 23.60 & 0.46 & 0.09 & 4.35 & 0.05 & 2.44 & 0.24 \\
5.1 & 24.28 & 1.34 & 0.12 & 6.39 & 0.15 & 3.35 & 0.50 \\
5.2 & 24.47 & 1.23 & 0.14 & 7.86 & 0.13 & 2.02 & 0.69 \\
5.3 & 24.34 & 1.09 & 0.14 & 7.54 & 0.12 & 1.57 & 0.69 \\
5.4 & 24.60 & 0.89 & 0.15 & 7.95 & 0.10 & 1.09 & 0.91 \\
\hline
\end{tabular}

Table 9.c. Emission line spectrum at solar metallicity (II)

\begin{tabular}{cccccccc}
\hline \hline Age & {$[\mathrm{NII}] 6584$} & [SII] 6716 & [SII] 6731 & [SIII] 9532 & [CII] $158 \mu \mathrm{m}$ & $\mathrm{EW}(\mathrm{H} \beta)$ & $T_{e}\left(\mathrm{~K}^{r}\right)$ \\
\hline 1.5 & 1.03 & 0.49 & 0.34 & 0.83 & 1.37 & 432.27 \\
2.0 & 1.05 & 0.49 & 0.34 & 0.76 & 1.55 & 357.44 & 6530 \\
2.5 & 1.05 & 0.48 & 0.33 & 0.76 & 1.58 & 346.11 & 6510 \\
3.0 & 1.06 & 0.49 & 0.34 & 0.68 & 1.79 & 295.59 & 6410 \\
3.5 & 1.10 & 0.59 & 0.41 & 0.49 & 2.26 & 166.80 & 6270 \\
4.0 & 1.31 & 0.83 & 0.57 & 0.64 & 2.03 & 58.65 & 6770 \\
4.5 & 1.82 & 1.21 & 0.84 & 1.22 & 2.03 & 189.46 & 7880 \\
5.0 & 1.55 & 0.94 & 0.65 & 1.19 & 1.52 & 267.97 & 7710 \\
5.1 & 2.25 & 1.61 & 1.11 & 1.33 & 2.70 & 213.74 & 8170 \\
5.2 & 2.85 & 2.16 & 1.49 & 1.06 & 3.52 & 116.71 & 8140 \\
5.3 & 2.83 & 2.19 & 1.51 & 0.95 & 3.64 & 96.23 & 8040 \\
5.4 & 3.07 & 2.57 & 1.77 & 0.84 & 4.58 & 73.22 & 7940 \\
\hline
\end{tabular}

Table 9.d. Emission line spectrum at twice solar metallicity (I)

\begin{tabular}{cccccccc}
\hline \hline Age & Ly $\alpha$ 1216 & HeII 1640 & MgII 2800 & [OII] 3727 & HeII 4686 & [OIII] 5007 & [OI] 6300 \\
\hline 1.5 & 22.46 & 0.00 & 0.01 & 0.91 & 0.00 & 0.17 & 0.05 \\
2.0 & 22.50 & 0.00 & 0.01 & 0.74 & 0.00 & 0.11 & 0.03 \\
2.5 & 22.51 & 0.00 & 0.01 & 0.71 & 0.00 & 0.09 & 0.02 \\
3.0 & 22.51 & 0.00 & 0.00 & 0.58 & 0.00 & 0.05 & 0.02 \\
3.5 & 22.53 & 0.00 & 0.00 & 0.44 & 0.00 & 0.01 & 0.02 \\
4.0 & 22.53 & 0.12 & 0.01 & 1.00 & 0.01 & 0.14 & 0.09 \\
4.5 & 22.81 & 0.67 & 0.06 & 3.45 & 0.07 & 0.96 & 0.35 \\
5.0 & 22.70 & 0.45 & 0.05 & 2.70 & 0.05 & 0.76 & 0.24 \\
5.1 & 22.96 & 1.28 & 0.06 & 4.27 & 0.14 & 1.43 & 0.47 \\
5.2 & 23.01 & 1.16 & 0.07 & 5.08 & 0.13 & 1.03 & 0.63 \\
5.3 & 22.97 & 1.02 & 0.07 & 4.64 & 0.11 & 0.79 & 0.63 \\
5.4 & 23.00 & 0.83 & 0.08 & 4.98 & 0.09 & 0.59 & 0.79 \\
\hline
\end{tabular}

Table 9.e. Emission line spectrum at twice solar metallicity (II)

\begin{tabular}{cccccccc}
\hline \hline Age & {$[\mathrm{NII}] 6584$} & [SII] 6716 & [SII] 6731 & [SIII] 9532 & [CII] 158 $\mu \mathrm{m}$ & $\mathrm{EW}(\mathrm{H} \beta)$ & $T_{e}(K)$ \\
\hline 1.5 & 0.67 & 0.36 & 0.25 & 0.50 & 2.30 & 449.91 & 4720 \\
2.0 & 0.64 & 0.33 & 0.23 & 0.47 & 2.61 & 372.17 & 4700 \\
2.5 & 0.63 & 0.33 & 0.22 & 0.47 & 2.66 & 359.67 & 4690 \\
3.0 & 0.60 & 0.31 & 0.22 & 0.43 & 2.97 & 306.33 & 4660 \\
3.5 & 0.57 & 0.35 & 0.24 & 0.32 & 3.72 & 171.67 & 4620 \\
4.0 & 0.82 & 0.58 & 0.40 & 0.39 & 3.36 & 58.89 & 4930 \\
4.5 & 1.77 & 1.21 & 0.83 & 0.96 & 3.33 & 184.61 & 6000 \\
5.0 & 1.43 & 0.92 & 0.63 & 0.87 & 2.64 & 265.22 & 5750 \\
5.1 & 2.24 & 1.62 & 1.11 & 1.20 & 4.45 & 212.01 & 6350 \\
5.2 & 2.83 & 2.20 & 1.51 & 1.07 & 5.73 & 114.24 & 6540 \\
5.3 & 2.73 & 2.18 & 1.50 & 0.95 & 5.94 & 94.25 & 6440 \\
5.4 & 2.99 & 2.54 & 1.75 & 0.87 & 7.24 & 71.18 & 6430 \\
\hline
\end{tabular}


TABLE 10

$M_{T}=0.4 \times 10^{5} M_{\odot}$, IMF PARAMETERS: $\alpha=2.35, m_{\text {low }}=0.85 M_{\odot}, m_{\mathrm{up}}=40 M_{\odot}$

Table 10.a. Parameters independent of metallicity

\begin{tabular}{ccccccccc}
\hline \hline Age & $\log L_{L y_{\alpha}}$ & $\log Q H$ & $\mathrm{QHe}_{\mathrm{QHH}}$ & $\mathrm{QO} / \mathrm{QH}$ & $\log u$ & $\log I_{H}$ & $\epsilon$ & $M_{H I I} / \mathrm{M}_{\odot}$ \\
\hline 1.5 & 40.36 & 50.86 & 0.15 & 0.03 & -3.47 & 38.58 & 0.011 & $0.18 \mathrm{E}+06$ \\
2.0 & 40.32 & 50.83 & 0.13 & 0.02 & -3.50 & 38.54 & 0.010 & $0.16 \mathrm{E}+06$ \\
2.5 & 40.34 & 50.85 & 0.13 & 0.02 & -3.48 & 38.56 & 0.011 & $0.17 \mathrm{E}+06$ \\
3.0 & 40.33 & 50.85 & 0.11 & 0.02 & -3.49 & 38.55 & 0.011 & $0.17 \mathrm{E}+06$ \\
3.5 & 40.25 & 50.79 & 0.05 & 0.01 & -3.54 & 38.50 & 0.009 & $0.15 \mathrm{E}+06$ \\
4.0 & 40.31 & 50.84 & 0.06 & 0.01 & -3.49 & 38.54 & 0.011 & $0.17 \mathrm{E}+06$ \\
4.5 & 40.19 & 50.73 & 0.05 & 0.01 & -3.61 & 38.43 & 0.008 & $0.12 \mathrm{E}+06$ \\
5.0 & 40.05 & 50.59 & 0.05 & 0.01 & -3.74 & 38.30 & 0.006 & $0.94 \mathrm{E}+05$ \\
5.1 & 40.06 & 50.60 & 0.05 & 0.01 & -3.74 & 38.31 & 0.006 & $0.92 \mathrm{E}+05$ \\
5.2 & 39.75 & 50.32 & 0.01 & 0.00 & -4.02 & 38.02 & 0.003 & $0.49 \mathrm{E}+05$ \\
5.3 & 39.74 & 50.31 & 0.01 & 0.00 & -4.03 & 38.01 & 0.003 & $0.48 \mathrm{E}+05$ \\
5.4 & 39.65 & 50.22 & 0.01 & 0.00 & -4.12 & 37.92 & 0.003 & $0.38 \mathrm{E}+05$ \\
\hline
\end{tabular}

Table 10.b. Emission line spectrum at solar metallicity (I)

\begin{tabular}{cccccccc}
\hline \hline Age & Ly $\alpha$ 1216 & HeII 1640 & MgII 2800 & [OII] 3727 & HeII 4686 & [OIII] 5007 & [OI] 6300 \\
\hline 1.5 & 22.98 & 0.00 & 0.04 & 2.98 & 0.00 & 0.18 & 0.08 \\
2.0 & 22.95 & 0.00 & 0.03 & 2.74 & 0.00 & 0.13 & 0.08 \\
2.5 & 22.96 & 0.00 & 0.03 & 2.75 & 0.00 & 0.14 & 0.07 \\
3.0 & 22.94 & 0.00 & 0.03 & 2.58 & 0.00 & 0.11 & 0.07 \\
3.5 & 22.90 & 0.00 & 0.02 & 1.87 & 0.00 & 0.02 & 0.06 \\
4.0 & 22.91 & 0.00 & 0.02 & 1.92 & 0.00 & 0.03 & 0.06 \\
4.5 & 22.90 & 0.00 & 0.02 & 1.82 & 0.00 & 0.02 & 0.07 \\
5.0 & 22.89 & 0.00 & 0.02 & 1.75 & 0.00 & 0.01 & 0.08 \\
5.1 & 22.89 & 0.00 & 0.02 & 1.75 & 0.00 & 0.01 & 0.08 \\
5.2 & 22.81 & 0.00 & 0.01 & 1.11 & 0.00 & 0.00 & 0.09 \\
5.3 & 22.81 & 0.00 & 0.01 & 1.13 & 0.00 & 0.00 & 0.09 \\
5.4 & 22.81 & 0.00 & 0.01 & 0.91 & 0.00 & 0.00 & 0.09 \\
\hline
\end{tabular}

Table 10.c. Emission line spectrum at solar metallicity (II)

\begin{tabular}{cccccccc}
\hline \hline Age & {$[\mathrm{NII}] 6584$} & [SII] 6716 & [SII] 6731 & [SIII] 9532 & [CII] 158 $\mu \mathrm{m}$ & $\left.\mathrm{EW}_{(\mathrm{H}}\right)$ & $T_{e}(\mathrm{~K})$ \\
\hline 1.5 & 1.47 & 0.87 & 0.60 & 0.59 & 2.19 & 285.13 \\
2.0 & 1.41 & 0.87 & 0.60 & 0.51 & 2.28 & 248.97 & 6700 \\
2.5 & 1.41 & 0.86 & 0.59 & 0.53 & 2.25 & 247.49 & 6710 \\
3.0 & 1.37 & 0.85 & 0.58 & 0.49 & 2.30 & 228.97 & 6630 \\
3.5 & 1.15 & 0.79 & 0.55 & 0.32 & 2.49 & 182.62 & 6300 \\
4.0 & 1.16 & 0.77 & 0.53 & 0.35 & 2.44 & 194.39 & 6320 \\
4.5 & 1.13 & 0.82 & 0.56 & 0.28 & 2.53 & 128.05 & 6260 \\
5.0 & 1.11 & 0.87 & 0.60 & 0.22 & 2.61 & 91.47 & 6220 \\
5.1 & 1.11 & 0.87 & 0.60 & 0.22 & 2.60 & 91.72 & 6230 \\
5.2 & 0.84 & 0.79 & 0.54 & 0.09 & 2.83 & 45.34 & 5830 \\
5.3 & 0.85 & 0.80 & 0.55 & 0.09 & 2.85 & 41.78 & 5840 \\
5.4 & 0.74 & 0.74 & 0.51 & 0.06 & 2.89 & 33.61 & 5640 \\
\hline
\end{tabular}

Table 10.d. Emission line spectrum at twice solar metallicity (I)

\begin{tabular}{cccccccc}
\hline \hline Age & Ly $\alpha$ 1216 & HeII 1640 & MgII 2800 & [OII] 3727 & HeII 4686 & [OIII] 5007 & [OI] 6300 \\
\hline 1.5 & 22.67 & 0.00 & 0.01 & 0.99 & 0.00 & 0.06 & 0.05 \\
2.0 & 22.65 & 0.00 & 0.01 & 0.89 & 0.00 & 0.04 & 0.05 \\
2.5 & 22.66 & 0.00 & 0.01 & 0.87 & 0.00 & 0.04 & 0.05 \\
3.0 & 22.64 & 0.00 & 0.01 & 0.80 & 0.00 & 0.04 & 0.04 \\
3.5 & 22.57 & 0.00 & 0.00 & 0.50 & 0.00 & 0.01 & 0.03 \\
4.0 & 22.57 & 0.00 & 0.00 & 0.52 & 0.00 & 0.01 & 0.03 \\
4.5 & 22.56 & 0.00 & 0.00 & 0.48 & 0.00 & 0.01 & 0.04 \\
5.0 & 22.57 & 0.00 & 0.00 & 0.46 & 0.00 & 0.00 & 0.04 \\
5.1 & 22.57 & 0.00 & 0.00 & 0.46 & 0.00 & 0.00 & 0.04 \\
5.2 & 22.45 & 0.00 & 0.00 & 0.19 & 0.00 & 0.00 & 0.03 \\
5.3 & 22.46 & 0.00 & 0.00 & 0.20 & 0.00 & 0.00 & 0.03 \\
5.4 & 22.44 & 0.00 & 0.00 & 0.14 & 0.00 & 0.00 & 0.03 \\
\hline
\end{tabular}

Table 10.e. Emission line spectrum at twice solar metallicity (II)

\begin{tabular}{cccccccc}
\hline \hline Age & {$[\mathrm{NII}] 6584$} & {$[\mathrm{SII}] 6716$} & {$[\mathrm{SII}] 6731$} & {$[\mathrm{SIII}] 9532$} & {$[\mathrm{CII}] 158 \mu \mathrm{m}$} & $\mathrm{EW}(\mathrm{H} \beta)$ & $T_{e}(K)$ \\
\hline 1.5 & 0.97 & 0.63 & 0.43 & 0.47 & 3.77 & 289.36 & 5160 \\
2.0 & 0.93 & 0.62 & 0.42 & 0.41 & 3.90 & 252.07 & 5110 \\
2.5 & 0.91 & 0.60 & 0.41 & 0.42 & 3.85 & 250.21 & 5060 \\
3.0 & 0.87 & 0.58 & 0.40 & 0.38 & 3.92 & 231.43 & 5020 \\
3.5 & 0.66 & 0.49 & 0.34 & 0.23 & 4.18 & 187.56 & 4760 \\
4.0 & 0.67 & 0.48 & 0.33 & 0.26 & 4.10 & 200.33 & 4780 \\
4.5 & 0.65 & 0.50 & 0.34 & 0.21 & 4.24 & 131.30 & 4740 \\
5.0 & 0.64 & 0.53 & 0.36 & 0.17 & 4.39 & 93.11 & 4720 \\
5.1 & 0.64 & 0.53 & 0.36 & 0.17 & 4.39 & 93.37 & 4720 \\
5.2 & 0.35 & 0.36 & 0.25 & 0.05 & 4.67 & 45.49 & 4220 \\
5.3 & 0.36 & 0.37 & 0.25 & 0.05 & 4.71 & 42.00 & 4240 \\
5.4 & 0.28 & 0.30 & 0.21 & 0.03 & 4.76 & 33.69 & 4020 \\
\hline
\end{tabular}


TABLE 11

$$
M_{T}=0.6 \times 10^{5} M_{\odot} \text {, IMF PARAMETERS: } \alpha=2.35, m_{\mathrm{low}}=0.85 M_{\odot}, m_{\mathrm{up}}=40 M_{\odot}
$$

Table 11.a. Parameters independent of metallicity

\begin{tabular}{ccccccccc}
\hline \hline Age & $\log L_{L y \alpha}$ & $\log Q H$ & QHe/QH & QO/QH & $\log u$ & $\log I_{H}$ & $\epsilon$ & $M_{H I I} / \mathrm{M}_{\odot}$ \\
\hline 1.5 & 40.54 & 51.04 & 0.15 & 0.03 & -3.29 & 38.76 & 0.017 & $0.27 \mathrm{E}+06$ \\
2.0 & 40.50 & 51.02 & 0.13 & 0.02 & -3.32 & 38.72 & 0.016 & $0.25 \mathrm{E}+06$ \\
2.5 & 40.52 & 51.03 & 0.13 & 0.02 & -3.30 & 38.74 & 0.016 & $0.26 \mathrm{E}+06$ \\
3.0 & 40.51 & 51.03 & 0.11 & 0.02 & -3.31 & 38.73 & 0.016 & $0.25 \mathrm{E}+06$ \\
3.5 & 40.43 & 50.97 & 0.05 & 0.01 & -3.36 & 38.67 & 0.014 & $0.23 \mathrm{E}+06$ \\
4.0 & 40.49 & 51.03 & 0.06 & 0.01 & -3.31 & 38.72 & 0.016 & $0.25 \mathrm{E}+06$ \\
4.5 & 40.37 & 50.91 & 0.05 & 0.01 & -3.42 & 38.61 & 0.012 & $0.20 \mathrm{E}+06$ \\
5.0 & 40.23 & 50.78 & 0.05 & 0.01 & -3.56 & 38.48 & 0.009 & $0.14 \mathrm{E}+06$ \\
5.1 & 40.24 & 50.78 & 0.05 & 0.01 & -3.55 & 38.48 & 0.009 & $0.15 \mathrm{E}+06$ \\
5.2 & 39.94 & 50.50 & 0.01 & 0.00 & -3.84 & 38.20 & 0.005 & $0.73 \mathrm{E}+05$ \\
5.3 & 39.93 & 50.49 & 0.01 & 0.00 & -3.85 & 38.20 & 0.005 & $0.71 \mathrm{E}+05$ \\
5.4 & 39.83 & 50.40 & 0.01 & 0.00 & -3.93 & 38.11 & 0.004 & $0.62 \mathrm{E}+05$ \\
\hline
\end{tabular}

Table 11.b. Emission line spectrum at solar metallicity (I)

\begin{tabular}{cccccccc}
\hline Age & Ly $\alpha$ 1216 & HeII 1640 & MgII 2800 & [OII] 3727 & HeII 4686 & [OIII] 5007 & [OI] 6300 \\
\hline 1.5 & 22.95 & 0.00 & 0.03 & 2.76 & 0.00 & 0.30 & 0.06 \\
2.0 & 22.94 & 0.00 & 0.03 & 2.57 & 0.00 & 0.22 & 0.06 \\
2.5 & 22.95 & 0.00 & 0.03 & 2.53 & 0.00 & 0.22 & 0.06 \\
3.0 & 22.93 & 0.00 & 0.03 & 2.43 & 0.00 & 0.19 & 0.05 \\
3.5 & 22.89 & 0.00 & 0.02 & 1.87 & 0.00 & 0.04 & 0.05 \\
4.0 & 22.89 & 0.00 & 0.02 & 1.90 & 0.00 & 0.04 & 0.05 \\
4.5 & 22.90 & 0.00 & 0.02 & 1.83 & 0.00 & 0.03 & 0.05 \\
5.0 & 22.89 & 0.00 & 0.02 & 1.79 & 0.00 & 0.02 & 0.06 \\
5.1 & 22.89 & 0.00 & 0.02 & 1.79 & 0.00 & 0.02 & 0.06 \\
5.2 & 22.82 & 0.00 & 0.01 & 1.20 & 0.00 & 0.00 & 0.08 \\
5.3 & 22.83 & 0.00 & 0.02 & 1.22 & 0.00 & 0.00 & 0.08 \\
5.4 & 22.81 & 0.00 & 0.01 & 1.03 & 0.00 & 0.00 & 0.08 \\
\hline
\end{tabular}

Table 11.c. Emission line spectrum at solar metallicity (II)

\begin{tabular}{cccccccc}
\hline \hline Age & [NII] 6584 & [SII] 6716 & [SII] 6731 & [SIII] 9532 & [CII] 158 $\mu \mathrm{m}$ & $\mathrm{EW}(\mathrm{H} \beta)$ & $T_{e}(K)$ \\
\hline 1.5 & 1.35 & 0.71 & 0.49 & 0.68 & 1.96 & 285.14 & 6750 \\
2.0 & 1.32 & 0.72 & 0.50 & 0.61 & 2.07 & 248.28 & 6660 \\
2.5 & 1.30 & 0.71 & 0.49 & 0.61 & 2.05 & 245.39 & 6620 \\
3.0 & 1.29 & 0.71 & 0.49 & 0.58 & 2.11 & 228.20 & 6600 \\
3.5 & 1.14 & 0.70 & 0.48 & 0.40 & 2.38 & 181.93 & 6300 \\
4.0 & 1.14 & 0.67 & 0.46 & 0.44 & 2.33 & 194.06 & 6310 \\
4.5 & 1.13 & 0.73 & 0.50 & 0.36 & 2.42 & 128.50 & 6270 \\
5.0 & 1.12 & 0.79 & 0.55 & 0.29 & 2.48 & 91.45 & 6250 \\
5.1 & 1.12 & 0.79 & 0.54 & 0.29 & 2.48 & 91.50 & 6250 \\
5.2 & 0.87 & 0.76 & 0.52 & 0.12 & 2.67 & 45.51 & 5900 \\
5.3 & 0.88 & 0.77 & 0.53 & 0.12 & 2.68 & 42.05 & 5910 \\
5.4 & 0.80 & 0.74 & 0.51 & 0.09 & 2.72 & 33.79 & 5760 \\
\hline
\end{tabular}

Table 11.d. Emission line spectrum at twice solar metallicity (I)

\begin{tabular}{cccccccc}
\hline \hline Age & Ly $\alpha$ 1216 & HeII 1640 & MgII 2800 & [OII] 3727 & HeII 4686 & [OIII] 5007 & [OI] 6300 \\
\hline 1.5 & 22.64 & 0.00 & 0.01 & 0.88 & 0.00 & 0.08 & 0.04 \\
2.0 & 22.63 & 0.00 & 0.01 & 0.78 & 0.00 & 0.06 & 0.04 \\
2.5 & 22.63 & 0.00 & 0.01 & 0.79 & 0.00 & 0.06 & 0.04 \\
3.0 & 22.62 & 0.00 & 0.00 & 0.71 & 0.00 & 0.05 & 0.03 \\
3.5 & 22.55 & 0.00 & 0.00 & 0.47 & 0.00 & 0.01 & 0.03 \\
4.0 & 22.56 & 0.00 & 0.00 & 0.48 & 0.00 & 0.01 & 0.03 \\
4.5 & 22.55 & 0.00 & 0.00 & 0.47 & 0.00 & 0.01 & 0.03 \\
5.0 & 22.56 & 0.00 & 0.00 & 0.46 & 0.00 & 0.01 & 0.03 \\
5.1 & 22.56 & 0.00 & 0.00 & 0.46 & 0.00 & 0.01 & 0.03 \\
5.2 & 22.49 & 0.00 & 0.00 & 0.23 & 0.00 & 0.00 & 0.03 \\
5.3 & 22.49 & 0.00 & 0.00 & 0.24 & 0.00 & 0.00 & 0.03 \\
5.4 & 22.46 & 0.00 & 0.00 & 0.18 & 0.00 & 0.00 & 0.03 \\
\hline
\end{tabular}

Table 11.e. Emission line spectrum at twice solar metallicity (II)

\begin{tabular}{cccccccc}
\hline \hline Age & {$[\mathrm{NII}] 6584$} & {$[\mathrm{SII}] 6716$} & [SII] 6731 & [SIII] 9532 & [CII] $158 \mu \mathrm{m}$ & $\mathrm{EW}(\mathrm{H} \beta)$ & $T_{e}(K)$ \\
\hline 1.5 & 0.85 & 0.50 & 0.35 & 0.50 & 3.36 & 289.72 & 5030 \\
2.0 & 0.82 & 0.50 & 0.34 & 0.45 & 3.54 & 251.76 & 4950 \\
2.5 & 0.81 & 0.49 & 0.34 & 0.45 & 3.49 & 250.75 & 4950 \\
3.0 & 0.78 & 0.47 & 0.33 & 0.42 & 3.59 & 231.34 & 4890 \\
3.5 & 0.62 & 0.42 & 0.29 & 0.28 & 3.95 & 186.10 & 4690 \\
4.0 & 0.62 & 0.40 & 0.28 & 0.30 & 3.86 & 199.70 & 4690 \\
4.5 & 0.62 & 0.44 & 0.30 & 0.25 & 4.01 & 131.50 & 4700 \\
5.0 & 0.63 & 0.48 & 0.33 & 0.21 & 4.16 & 93.31 & 4710 \\
5.1 & 0.63 & 0.48 & 0.33 & 0.21 & 4.15 & 93.36 & 4710 \\
5.2 & 0.39 & 0.37 & 0.25 & 0.07 & 4.40 & 45.77 & 4270 \\
5.3 & 0.40 & 0.38 & 0.26 & 0.08 & 4.43 & 42.28 & 4290 \\
5.4 & 0.33 & 0.33 & 0.23 & 0.05 & 4.47 & 33.80 & 4130 \\
\hline
\end{tabular}


TABLE 12

$M_{T}=0.4 \times 10^{5} M_{\odot}$, IMF PARAMETERS: $\alpha=2.8, m_{\mathrm{low}}=0.85 M_{\odot}, m_{\mathrm{up}}=120 M_{\odot}$

Table 12.a. Parameters independent of metallicity

\begin{tabular}{ccccccccc}
\hline Age & $\log L_{L y_{\alpha}}$ & $\log Q H$ & $\mathrm{QHe} / \mathrm{QH}$ & $\mathrm{QO} / \mathrm{QH}$ & $\log u$ & $\log I_{H}$ & $\epsilon$ & $M_{H I I} / \mathrm{M}_{\odot}$ \\
\hline 1.5 & 40.20 & 50.68 & 0.20 & 0.05 & -3.65 & 38.40 & 0.007 & $0.12 \mathrm{E}+06$ \\
2.0 & 40.14 & 50.65 & 0.15 & 0.03 & -3.69 & 38.35 & 0.007 & $0.11 \mathrm{E}+06$ \\
2.5 & 40.16 & 50.66 & 0.15 & 0.03 & -3.67 & 38.38 & 0.007 & $0.11 \mathrm{E}+06$ \\
3.0 & 40.08 & 50.60 & 0.11 & 0.02 & -3.74 & 38.31 & 0.006 & $0.93 \mathrm{E}+05$ \\
3.5 & 39.95 & 50.49 & 0.05 & 0.01 & -3.84 & 38.20 & 0.005 & $0.76 \mathrm{E}+05$ \\
4.0 & 40.15 & 50.52 & 0.27 & 0.18 & -3.81 & 38.25 & 0.005 & $0: 80 \mathrm{E}+05$ \\
4.5 & 40.12 & 50.49 & 0.32 & 0.20 & -3.85 & 38.21 & 0.005 & $0.71 \mathrm{E}+05$ \\
5.0 & 40.21 & 50.58 & 0.37 & 0.19 & -3.75 & 38.30 & 0.006 & $0.92 \mathrm{E}+05$ \\
5.1 & 40.19 & 50.47 & 0.43 & 0.30 & -3.87 & 38.20 & 0.004 & $0.70 \mathrm{E}+05$ \\
5.2 & 39.91 & 50.19 & 0.42 & 0.30 & -4.14 & 37.92 & 0.002 & $0.37 \mathrm{E}+05$ \\
5.3 & 39.84 & 50.14 & 0.38 & 0.27 & -4.20 & 37.87 & 0.002 & $0.31 \mathrm{E}+05$ \\
5.4 & 39.73 & 50.03 & 0.36 & 0.27 & -4.31 & 37.75 & 0.002 & $0.25 \mathrm{E}+05$ \\
\hline
\end{tabular}

Table 12.b. Emission line spectrum at solar metallicity (I)

\begin{tabular}{cccccccc}
\hline \hline Age & Ly $\alpha$ 1216 & HeII 1640 & MgII 2800 & [OII] 3727 & HeII 4686 & [OIII] 5007 & [OI] 6300 \\
\hline 1.5 & 23.04 & 0.00 & 0.06 & 3.66 & 0.00 & 0.19 & 0.16 \\
2.0 & 22.99 & 0.00 & 0.04 & 3.01 & 0.00 & 0.09 & 0.12 \\
2.5 & 22.97 & 0.00 & 0.04 & 3.03 & 0.00 & 0.09 & 0.11 \\
3.0 & 22.94 & 0.00 & 0.03 & 2.61 & 0.00 & 0.05 & 0.10 \\
3.5 & 22.88 & 0.00 & 0.02 & 1.78 & 0.00 & 0.01 & 0.09 \\
4.0 & 23.44 & 0.43 & 0.10 & 5.27 & 0.05 & 0.32 & 0.56 \\
4.5 & 23.65 & 0.27 & 0.13 & 5.79 & 0.03 & 0.34 & 0.64 \\
5.0 & 23.69 & 0.29 & 0.14 & 6.34 & 0.03 & 0.49 & 0.58 \\
5.1 & 24.39 & 0.74 & 0.17 & 7.90 & 0.08 & 0.47 & 1.04 \\
5.2 & 24.39 & 0.59 & 0.19 & 7.25 & 0.06 & 0.17 & 1.36 \\
5.3 & 24.06 & 0.48 & 0.18 & 6.43 & 0.05 & 0.11 & 1.28 \\
5.4 & 23.91 & 0.35 & 0.17 & 5.78 & 0.04 & 0.07 & 1.36 \\
\hline
\end{tabular}

Table 12.c. Emission line spectrum at solar metallicity (II)

\begin{tabular}{cccccccc}
\hline \hline Age & {$[\mathrm{NII}] 6584$} & {$[\mathrm{SII}] 6716$} & {$[\mathrm{SII}] 6731$} & [SIII] 9532 & [CII] $158 \mu \mathrm{m}$ & $\left.\mathrm{EW}_{(\mathrm{H}}\right)$ & $T_{e}(\mathrm{~K})$ \\
\hline 1.5 & 1.71 & 1.12 & 0.77 & 0.59 & 2.44 & 316.56 & 7030 \\
2.0 & 1.52 & 1.04 & 0.71 & 0.46 & 2.47 & 265.50 & 6790 \\
2.5 & 1.53 & 1.03 & 0.71 & 0.46 & 2.45 & 265.92 & 6810 \\
3.0 & 1.40 & 1.01 & 0.70 & 0.36 & 2.55 & 204.65 & 6620 \\
3.5 & 1.12 & 0.92 & 0.63 & 0.20 & 2.70 & 137.55 & 6240 \\
4.0 & 2.33 & 2.02 & 1.39 & 0.50 & 4.00 & 100.01 & 7440 \\
4.5 & 2.50 & 2.13 & 1.47 & 0.55 & 3.84 & 135.79 & 7620 \\
5.0 & 2.59 & 1.98 & 1.37 & 0.73 & 3.35 & 191.88 & 7790 \\
5.1 & 3.18 & 2.86 & 1.97 & 0.65 & 5.13 & 156.64 & 7840 \\
5.2 & 3.20 & 3.35 & 2.31 & 0.44 & 6.32 & 81.01 & 7720 \\
5.3 & 2.95 & 3.21 & 2.21 & 0.38 & 6.38 & 68.90 & 7560 \\
5.4 & 2.80 & 3.29 & 2.26 & 0.31 & 7.23 & 52.63 & 7350 \\
\hline
\end{tabular}

Table 12.d. Emission line spectrum at twice solar metallicity (I)

\begin{tabular}{cccccccc}
\hline \hline Age & Ly $\alpha$ 1216 & HeII 1640 & MgII 2800 & [OII] 3727 & HeII 4686 & [OIII] 5007 & [OI] 6300 \\
\hline 1.5 & 22.74 & 0.00 & 0.02 & 1.45 & 0.00 & 0.08 & 0.11 \\
2.0 & 22.68 & 0.00 & 0.01 & 1.07 & 0.00 & 0.04 & 0.08 \\
2.5 & 22.68 & 0.00 & 0.01 & 1.05 & 0.00 & 0.04 & 0.07 \\
3.0 & 22.64 & 0.00 & 0.01 & 0.86 & 0.00 & 0.02 & 0.06 \\
3.5 & 22.57 & 0.00 & 0.00 & 0.48 & 0.00 & 0.00 & 0.04 \\
4.0 & 22.80 & 0.39 & 0.04 & 2.50 & 0.04 & 0.16 & 0.40 \\
4.5 & 22.83 & 0.25 & 0.05 & 2.95 & 0.03 & 0.17 & 0.49 \\
5.0 & 22.86 & 0.27 & 0.05 & 3.35 & 0.03 & 0.25 & 0.46 \\
5.1 & 22.95 & 0.69 & 0.08 & 4.72 & 0.08 & 0.29 & 0.86 \\
5.2 & 22.90 & 0.54 & 0.08 & 4.05 & 0.06 & 0.11 & 1.01 \\
5.3 & 22.85 & 0.44 & 0.06 & 3.34 & 0.05 & 0.07 & 0.89 \\
5.4 & 22.81 & 0.32 & 0.05 & 2.78 & 0.04 & 0.04 & 0.87 \\
\hline
\end{tabular}

Table 12.e. Emission line spectrum at twice solar metallicity (II)

\begin{tabular}{cccccccc}
\hline \hline Age & {$[\mathrm{NII}] 6584$} & {$[\mathrm{SII}] 6716$} & [SII] 6731 & [SIII] 9532 & [CII] 158 $\mu \mathrm{m}$ & $\mathrm{EW}(\mathrm{H} \beta)$ & $T_{e}(K)$ \\
\hline 1.5 & 1.27 & 0.89 & 0.61 & 0.52 & 4.24 & 322.28 & 5430 \\
2.0 & 1.07 & 0.77 & 0.53 & 0.39 & 4.29 & 269.68 & 5210 \\
2.5 & 1.05 & 0.76 & 0.52 & 0.39 & 4.24 & 268.46 & 5210 \\
3.0 & 0.94 & 0.72 & 0.49 & 0.30 & 4.39 & 209.64 & 5100 \\
3.5 & 0.66 & 0.56 & 0.39 & 0.15 & 4.57 & 140.47 & 4740 \\
4.0 & 1.92 & 1.74 & 1.20 & 0.48 & 6.58 & 98.41 & 5840 \\
4.5 & 2.16 & 1.93 & 1.32 & 0.55 & 6.45 & 134.49 & 6020 \\
5.0 & 2.28 & 1.84 & 1.26 & 0.72 & 5.76 & 192.18 & 6140 \\
5.1 & 3.05 & 2.79 & 1.92 & 0.71 & 8.26 & 153.93 & 6370 \\
5.2 & 2.92 & 3.10 & 2.12 & 0.49 & 10.30 & 79.82 & 6180 \\
5.3 & 2.56 & 2.82 & 1.94 & 0.40 & 10.32 & 68.06 & 6020 \\
5.4 & 2.29 & 2.73 & 1.87 & 0.32 & 11.34 & 51.29 & 5810 \\
\hline
\end{tabular}


TABLE 13

$M_{T}=0.6 \times 10^{5} M_{\odot}$, IMF PARAMETERS: $\alpha=2.8, m_{\mathrm{low}}=0.85 M_{\odot}, m_{\mathrm{up}}=120 M_{\odot}$

Table 13.a. Parameters independent of metallicity

\begin{tabular}{ccccccccc}
\hline \hline Age & $\log L_{L_{\alpha}}$ & $\log Q H$ & $\mathrm{QHe} / \mathrm{QH}$ & $\mathrm{QO} / \mathrm{QH}$ & $\log u$ & $\log I_{H}$ & $\epsilon$ & $M_{H I I} / \mathrm{M}_{\odot}$ \\
\hline 1.5 & 40.55 & 51.03 & 0.22 & 0.05 & -3.31 & 38.74 & 0.016 & $0.25 \mathrm{E}+06$ \\
2.0 & 40.49 & 50.99 & 0.16 & 0.03 & -3.34 & 38.70 & 0.015 & $0.24 \mathrm{E}+06$ \\
2.5 & 40.47 & 50.99 & 0.13 & 0.02 & -3.35 & 38.69 & 0.015 & $0.23 \mathrm{E}+06$ \\
3.0 & 40.45 & 50.96 & 0.14 & 0.03 & -3.38 & 38.67 & 0.014 & $0.21 \mathrm{E}+06$ \\
3.5 & 40.59 & 50.96 & 0.30 & 0.19 & -3.38 & 38.68 & 0.014 & $0.21 \mathrm{E}+06$ \\
4.0 & 40.48 & 50.79 & 0.36 & 0.25 & -3.55 & 38.53 & 0.009 & $0.15 \mathrm{E}+06$ \\
4.5 & 40.32 & 50.69 & 0.33 & 0.21 & -3.65 & 38.41 & 0.007 & $0.11 \mathrm{E}+06$ \\
5.0 & 40.44 & 50.80 & 0.38 & 0.19 & -3.53 & 38.52 & 0.010 & $0.15 \mathrm{E}+06$ \\
5.1 & 40.41 & 50.68 & 0.45 & 0.31 & -3.66 & 38.42 & 0.007 & $0.11 \mathrm{E}+06$ \\
5.2 & 40.15 & 50.41 & 0.44 & 0.32 & -3.92 & 38.15 & 0.004 & $0.62 \mathrm{E}+05$ \\
5.3 & 40.07 & 50.36 & 0.40 & 0.29 & -3.98 & 38.09 & 0.003 & $0.53 \mathrm{E}+05$ \\
5.4 & 39.96 & 50.25 & 0.38 & 0.28 & -4.09 & 37.98 & 0.003 & $0.42 \mathrm{E}+05$ \\
\hline
\end{tabular}

Table 13.b. Emission line spectrum at solar metallicity (I)

\begin{tabular}{cccccccc}
\hline \hline Age & Ly $\alpha$ 1216 & HeII 1640 & MgII 2800 & [OII] 3727 & HeII 4686 & [OIII] 5007 & [OI] 6300 \\
\hline 1.5 & 23.04 & 0.00 & 0.05 & 3.32 & 0.00 & 0.55 & 0.11 \\
2.0 & 22.97 & 0.00 & 0.04 & 2.87 & 0.00 & 0.29 & 0.07 \\
2.5 & 22.95 & 0.00 & 0.03 & 2.62 & 0.00 & 0.20 & 0.06 \\
3.0 & 22.95 & 0.00 & 0.03 & 2.73 & 0.00 & 0.24 & 0.07 \\
3.5 & 23.53 & 0.51 & 0.09 & 4.72 & 0.06 & 1.19 & 0.36 \\
4.0 & 23.90 & 0.81 & 0.12 & 6.37 & 0.09 & 1.11 & 0.61 \\
4.5 & 23.73 & 0.37 & 0.13 & 5.87 & 0.04 & 0.68 & 0.55 \\
5.0 & 23.71 & 0.35 & 0.13 & 6.11 & 0.04 & 0.98 & 0.45 \\
5.1 & 24.40 & 0.94 & 0.15 & 8.01 & 0.10 & 1.06 & 0.83 \\
5.2 & 24.60 & 0.79 & 0.18 & 8.21 & 0.09 & 0.42 & 1.15 \\
5.3 & 24.32 & 0.67 & 0.17 & 7.35 & 0.07 & 0.30 & 1.10 \\
5.4 & 24.24 & 0.50 & 0.17 & 6.83 & 0.05 & 0.18 & 1.23 \\
\hline
\end{tabular}

Table 13.c. Emission line spectrum at solar metallicity (II)

\begin{tabular}{cccccccc}
\hline \hline Age & [NII] 6584 & [SII] 6716 & [SII] 6731 & [SII] 9532 & [CII] 158 $\mu \mathrm{m}$ & $\mathrm{EW}(\mathrm{H} \beta)$ & $T_{e}(K)$ \\
\hline 1.5 & 1.47 & 0.80 & 0.55 & 0.80 & 1.85 & 387.85 & 6970 \\
2.0 & 1.39 & 0.76 & 0.53 & 0.67 & 2.02 & 317.67 & 6770 \\
2.5 & 1.34 & 0.75 & 0.51 & 0.60 & 2.10 & 285.84 & 6670 \\
3.0 & 1.38 & 0.78 & 0.54 & 0.60 & 2.10 & 245.11 & 6720 \\
3.5 & 1.96 & 1.38 & 0.95 & 0.84 & 2.72 & 230.37 & 7460 \\
4.0 & 2.56 & 2.02 & 1.39 & 0.82 & 3.80 & 107.98 & 7740 \\
4.5 & 2.43 & 1.89 & 1.31 & 0.70 & 3.36 & 136.40 & 7670 \\
5.0 & 2.39 & 1.64 & 1.13 & 0.91 & 2.71 & 208.14 & 7820 \\
5.1 & 3.06 & 2.47 & 1.71 & 0.86 & 4.28 & 171.12 & 7990 \\
5.2 & 3.32 & 3.06 & 2.11 & 0.62 & 5.30 & 90.11 & 7930 \\
5.3 & 3.10 & 2.97 & 2.05 & 0.53 & 5.37 & 76.61 & 7790 \\
5.4 & 3.02 & 3.14 & 2.16 & 0.44 & 6.24 & 58.86 & 7590 \\
\hline
\end{tabular}

Table 13.d. Emission line spectrum at twice solar metallicity (I)

\begin{tabular}{cccccccc}
\hline \hline Age & Ly $\alpha 1216$ & HeII 1640 & MgII 2800 & [OII] 3727 & HeII 4686 & [OIII] 5007 & [OI] 6300 \\
\hline 1.5 & 22.66 & 0.00 & 0.01 & 1.30 & 0.00 & 0.17 & 0.08 \\
2.0 & 22.65 & 0.00 & 0.01 & 0.96 & 0.00 & 0.08 & 0.05 \\
2.5 & 22.65 & 0.00 & 0.01 & 0.80 & 0.00 & 0.05 & 0.04 \\
3.0 & 22.65 & 0.00 & 0.01 & 0.86 & 0.00 & 0.07 & 0.04 \\
3.5 & 22.75 & 0.47 & 0.04 & 2.39 & 0.05 & 0.41 & 0.30 \\
4.0 & 22.88 & 0.76 & 0.05 & 3.63 & 0.08 & 0.53 & 0.52 \\
4.5 & 22.85 & 0.34 & 0.05 & 3.10 & 0.04 & 0.32 & 0.45 \\
5.0 & 22.85 & 0.33 & 0.05 & 3.28 & 0.04 & 0.43 & 0.38 \\
5.1 & 22.99 & 0.89 & 0.07 & 5.07 & 0.10 & 0.59 & 0.75 \\
5.2 & 22.98 & 0.74 & 0.08 & 4.96 & 0.08 & 0.27 & 0.96 \\
5.3 & 22.93 & 0.62 & 0.07 & 4.22 & 0.07 & 0.18 & 0.89 \\
5.4 & 22.88 & 0.46 & 0.07 & 3.70 & 0.05 & 0.11 & 0.93 \\
\hline
\end{tabular}

Table 13.e. Emission line spectrum at twice solar metallicity (II)

\begin{tabular}{cccccccc}
\hline \hline Age & {$[\mathrm{NII}] 6584$} & {$[\mathrm{SII}] 6716$} & {$[\mathrm{SII}] 6731$} & {$[\mathrm{SIII}] 9532$} & {$[\mathrm{CII}] 158 \mu \mathrm{m}$} & $\mathrm{EW}(\mathrm{H} \beta)$ & $T_{e}(K)$ \\
\hline 1.5 & 1.04 & 0.62 & 0.43 & 0.61 & 3.23 & 400.49 & 5260 \\
2.0 & 0.91 & 0.55 & 0.38 & 0.51 & 3.45 & 325.71 & 5100 \\
2.5 & 0.84 & 0.52 & 0.35 & 0.45 & 3.57 & 290.03 & 4980 \\
3.0 & 0.86 & 0.54 & 0.37 & 0.45 & 3.58 & 247.93 & 5020 \\
3.5 & 1.61 & 1.21 & 0.83 & 0.69 & 4.36 & 231.69 & 5770 \\
4.0 & 2.35 & 1.92 & 1.32 & 0.80 & 6.08 & 104.68 & 6160 \\
4.5 & 2.12 & 1.75 & 1.20 & 0.67 & 5.56 & 135.16 & 6060 \\
5.0 & 2.10 & 1.54 & 1.06 & 0.85 & 4.66 & 208.74 & 6130 \\
5.1 & 3.03 & 2.51 & 1.72 & 0.91 & 6.90 & 167.21 & 6490 \\
5.2 & 3.20 & 3.02 & 2.07 & 0.68 & 8.68 & 88.15 & 6430 \\
5.3 & 2.90 & 2.84 & 1.95 & 0.57 & 8.85 & 74.27 & 6270 \\
5.4 & 2.72 & 2.87 & 1.97 & 0.47 & 9.90 & 56.40 & 6100 \\
\hline
\end{tabular}


TABLE 14

$M_{T}=4 \times 10^{5} M_{\odot}$, IMF PARAMETERS: $\alpha=2.8, m_{\text {low }}=0.85 M_{\odot}, m_{\mathrm{up}}=120 M_{\odot}$

Table 14.a. Parameters independent of metallicity

\begin{tabular}{ccccccccc}
\hline \hline Age & $\log L_{L y_{\alpha}}$ & $\log Q H$ & $\mathrm{QHe} / \mathrm{QH}$ & $\mathrm{QO} / \mathrm{QH}$ & $\log u$ & $\log I_{H_{\beta}}$ & $\epsilon$ & $M_{H I I} / \mathrm{M}_{\odot}$ \\
\hline 1.5 & 41.47 & 51.95 & 0.23 & 0.05 & -2.39 & 39.66 & 0.136 & $0.21 \mathrm{E}+07$ \\
2.0 & 41.40 & 51.91 & 0.16 & 0.03 & -2.43 & 39.61 & 0.123 & $0.19 \mathrm{E}+07$ \\
2.5 & 41.39 & 51.90 & 0.13 & 0.02 & -2.43 & 39.60 & 0.121 & $0.19 \mathrm{E}+07$ \\
3.0 & 41.41 & 51.90 & 0.17 & 0.04 & -2.44 & 39.61 & 0.121 & $0.18 \mathrm{E}+07$ \\
3.5 & 41.57 & 51.88 & 0.37 & 0.25 & -2.46 & 39.60 & 0.116 & $0.17 \mathrm{E}+07$ \\
4.0 & 41.40 & 51.68 & 0.40 & 0.29 & -2.66 & 39.40 & 0.072 & $0.11 \mathrm{E}+07$ \\
4.5 & 41.23 & 51.57 & 0.37 & 0.23 & -2.77 & 39.29 & 0.057 & $0.85 \mathrm{E}+06$ \\
5.0 & 41.26 & 51.63 & 0.37 & 0.19 & -2.70 & 39.35 & 0.066 & $0.10 \mathrm{E}+07$ \\
5.1 & 41.24 & 51.51 & 0.44 & 0.31 & -2.83 & 39.24 & 0.049 & $0.75 \mathrm{E}+06$ \\
5.2 & 40.97 & 51.25 & 0.43 & 0.31 & -3.09 & 38.99 & 0.027 & $0.41 \mathrm{E}+06$ \\
5.3 & 40.90 & 51.20 & 0.39 & 0.28 & -3.14 & 38.93 & 0.024 & $0.37 \mathrm{E}+06$ \\
5.4 & 40.79 & 51.08 & 0.38 & 0.28 & -3.26 & 38.82 & 0.018 & $0.28 \mathrm{E}+06$ \\
\hline
\end{tabular}

Table 14.b. Emission line spectrum at solar metallicity (I)

\begin{tabular}{cccccccc}
\hline \hline Age & Ly $\alpha 1216$ & HeII 1640 & MgII 2800 & [OII] 3727 & HeII 4686 & [OIII] 5007 & [OI] 6300 \\
\hline 1.5 & 22.79 & 0.00 & 0.02 & 1.09 & 0.00 & 1.07 & 0.03 \\
2.0 & 22.78 & 0.00 & 0.01 & 1.14 & 0.00 & 0.64 & 0.01 \\
2.5 & 22.77 & 0.00 & 0.01 & 1.23 & 0.00 & 0.44 & 0.01 \\
3.0 & 22.73 & 0.00 & 0.01 & 0.99 & 0.00 & 0.68 & 0.02 \\
3.5 & 23.49 & 1.06 & 0.05 & 2.12 & 0.12 & 4.40 & 0.11 \\
4.0 & 23.67 & 1.33 & 0.07 & 2.89 & 0.15 & 4.61 & 0.19 \\
4.5 & 23.66 & 0.66 & 0.08 & 3.23 & 0.07 & 3.26 & 0.18 \\
5.0 & 23.46 & 0.46 & 0.07 & 2.87 & 0.05 & 2.96 & 0.14 \\
5.1 & 23.85 & 1.36 & 0.08 & 3.84 & 0.15 & 4.46 & 0.25 \\
5.2 & 23.99 & 1.32 & 0.10 & 5.03 & 0.14 & 3.30 & 0.37 \\
5.3 & 23.84 & 1.17 & 0.09 & 4.81 & 0.13 & 2.72 & 0.36 \\
5.4 & 24.05 & 0.99 & 0.10 & 5.54 & 0.11 & 2.31 & 0.51 \\
\hline
\end{tabular}

Table 14.c. Emission line spectrum at solar metallicity (II)

\begin{tabular}{cccccccc}
\hline \hline Age & {$[\mathrm{NII}] 6584$} & {$[\mathrm{SII}] 6716$} & [SII] 6731 & [SIII] 9532 & [CII] 158 $\mu \mathrm{m}$ & $\mathrm{EW}(\mathrm{H} \beta)$ & $T_{e}(\mathrm{~K})$ \\
\hline 1.5 & 0.41 & 0.18 & 0.13 & 0.71 & 0.60 & 437.33 & 5870 \\
2.0 & 0.51 & 0.17 & 0.12 & 0.68 & 0.90 & 339.06 & 5770 \\
2.5 & 0.59 & 0.18 & 0.13 & 0.67 & 1.11 & 300.87 & 5810 \\
3.0 & 0.44 & 0.17 & 0.12 & 0.61 & 0.80 & 280.78 & 5640 \\
3.5 & 0.69 & 0.44 & 0.31 & 1.36 & 0.85 & 253.47 & 7600 \\
4.0 & 0.99 & 0.68 & 0.47 & 1.47 & 1.30 & 100.17 & 7830 \\
4.5 & 1.09 & 0.70 & 0.48 & 1.23 & 1.21 & 151.16 & 7490 \\
5.0 & 0.94 & 0.56 & 0.39 & 1.20 & 0.87 & 203.12 & 7330 \\
5.1 & 1.30 & 0.90 & 0.62 & 1.47 & 1.58 & 163.85 & 7970 \\
5.2 & 1.81 & 1.29 & 0.89 & 1.26 & 2.21 & 89.62 & 7970 \\
5.3 & 1.81 & 1.30 & 0.90 & 1.14 & 2.35 & 76.48 & 7800 \\
5.4 & 2.14 & 1.62 & 1.12 & 1.06 & 3.06 & 58.92 & 7810 \\
\hline
\end{tabular}

Table 14.d. Emission line spectrum at twice solar metallicity (I)

\begin{tabular}{|c|c|c|c|c|c|c|c|}
\hline Age & Ly $\alpha 1216$ & HeII 1640 & MgII 2800 & [OII] 3727 & HeII 4686 & [OIII] 5007 & [OI] 6300 \\
\hline 1.5 & 21.97 & 0.00 & 0.01 & 0.46 & 0.00 & 0.16 & 0.02 \\
\hline 2.0 & 22.07 & 0.00 & 0.00 & 0.37 & 0.00 & 0.07 & 0.01 \\
\hline 2.5 & 22.13 & 0.00 & 0.00 & 0.37 & 0.00 & 0.03 & 0.01 \\
\hline 3.0 & 21.97 & 0.00 & 0.00 & 0.35 & 0.00 & 0.08 & 0.01 \\
\hline 3.5 & 21.73 & 2.89 & 0.00 & 0.00 & 0.33 & 0.00 & 0.00 \\
\hline 4.0 & 21.80 & 2.86 & 0.00 & 0.00 & 0.32 & 0.01 & 0.00 \\
\hline 4.5 & 22.54 & 0.62 & 0.04 & 2.14 & 0.07 & 0.80 & 0.19 \\
\hline 5.0 & 22.45 & 0.44 & 0.03 & 1.75 & 0.05 & 0.71 & 0.14 \\
\hline 5.1 & 22.75 & 1.32 & 0.04 & 2.77 & 0.15 & 1.38 & 0.27 \\
\hline 5.2 & 22.84 & 1.26 & 0.05 & 3.31 & 0.14 & 1.10 & 0.36 \\
\hline 5.3 & 22.81 & 1.12 & 0.04 & 3.04 & 0.12 & 0.89 & 0.35 \\
\hline 5.4 & 22.88 & 0.94 & 0.05 & 3.49 & 0.10 & 0.83 & 0.47 \\
\hline \multicolumn{8}{|c|}{ Table 14.e. Emission line spectrum at twice solar metallicity (II) } \\
\hline Age & [NII] 6584 & [SII] 6716 & [SII] 6731 & [SIII] 9532 & [CII] $158 \mu \mathrm{m}$ & $\mathrm{EW}\left(\mathrm{H}_{\beta}\right)$ & $T_{e}(K)$ \\
\hline 1.5 & 0.28 & 0.13 & 0.09 & 0.27 & 0.91 & 465.80 & 3640 \\
\hline 2.0 & 0.29 & 0.11 & 0.08 & 0.29 & 1.38 & 358.59 & 3720 \\
\hline 2.5 & 0.33 & 0.11 & 0.08 & 0.31 & 1.75 & 316.88 & 3860 \\
\hline 3.0 & 0.26 & 0.11 & 0.08 & 0.23 & 1.20 & 292.11 & 3520 \\
\hline 3.5 & 0.00 & 0.00 & 0.00 & 0.01 & 0.24 & 35.11 & 2120 \\
\hline 4.0 & 0.00 & 0.00 & 0.00 & 0.03 & 0.39 & 10.03 & 2350 \\
\hline 4.5 & 1.02 & 0.65 & 0.45 & 0.69 & 1.99 & 151.69 & 5270 \\
\hline 5.0 & 0.84 & 0.51 & 0.35 & 0.64 & 1.51 & 205.80 & 5060 \\
\hline 5.1 & 1.33 & 0.89 & 0.61 & 1.03 & 2.67 & 161.38 & 5790 \\
\hline 5.2 & 1.74 & 1.25 & 0.86 & 0.98 & 3.69 & 87.48 & 6020 \\
\hline 5.3 & 1.69 & 1.25 & 0.86 & 0.88 & 3.87 & 73.94 & 5920 \\
\hline 5.4 & 2.00 & 1.55 & 1.07 & 0.89 & 4.90 & 56.79 & 6060 \\
\hline
\end{tabular}


TABLE 15

$M_{T}=4 \times 10^{5} M_{\odot}$, IMF PARAMETERS: $\alpha=3.35, m_{\mathrm{low}}=0.85 M_{\odot}, m_{\mathrm{up}}=120 M_{\odot}$

Table 15.a. Parameters independent of metallicity

\begin{tabular}{ccccccccc}
\hline \hline Age & $\log L_{L_{y}}$ & $\log Q H$ & $\mathrm{QHe} / \mathrm{QH}$ & $\mathrm{QO} / \mathrm{QH}$ & $\log u$ & $\log I_{H_{\beta}}$ & $\epsilon$ & $M_{H I I} / \mathrm{M}_{\odot}$ \\
\hline 1.5 & 40.62 & 51.11 & 0.20 & 0.05 & -3.23 & 38.81 & 0.019 & $0.30 \mathrm{E}+06$ \\
2.0 & 40.56 & 51.07 & 0.15 & 0.03 & -3.27 & 38.78 & 0.018 & $0.27 \mathrm{E}+06$ \\
2.5 & 40.56 & 51.07 & 0.13 & 0.02 & -3.26 & 38.78 & 0.018 & $0.29 \mathrm{E}+06$ \\
3.0 & 40.51 & 51.03 & 0.12 & 0.02 & -3.31 & 38.73 & 0.016 & $0.25 \mathrm{E}+06$ \\
3.5 & 40.50 & 50.98 & 0.13 & 0.07 & -3.36 & 38.69 & 0.014 & $0.22 \mathrm{E}+06$ \\
4.0 & 40.57 & 50.93 & 0.27 & 0.18 & -3.41 & 38.66 & 0.013 & $0.19 \mathrm{E}+06$ \\
4.5 & 40.51 & 50.89 & 0.29 & 0.18 & -3.45 & 38.61 & 0.012 & $0.18 \mathrm{E}+06$ \\
5.0 & 40.58 & 50.97 & 0.33 & 0.16 & -3.37 & 38.69 & 0.014 & $0.22 \mathrm{E}+06$ \\
5.1 & 40.56 & 50.86 & 0.38 & 0.26 & -3.48 & 38.60 & 0.011 & $0.17 \mathrm{E}+06$ \\
5.2 & 40.30 & 50.60 & 0.37 & 0.27 & -3.74 & 38.34 & 0.006 & $0.91 \mathrm{E}+05$ \\
5.3 & 40.23 & 50.56 & 0.33 & 0.24 & -3.78 & 38.29 & 0.005 & $0.85 \mathrm{E}+05$ \\
5.4 & 40.12 & 50.45 & 0.31 & 0.23 & -3.89 & 38.18 & 0.004 & $0.65 \mathrm{E}+05$ \\
\hline
\end{tabular}

Table 15.b. Emission line spectrum at solar metallicity (I)

\begin{tabular}{cccccccc}
\hline \hline Age & Ly $\alpha$ 1216 & HeII 1640 & MgII 2800 & [OII] 3727 & HeII 4686 & [OIII] 5007 & [OI] 6300 \\
\hline 1.5 & 23.01 & 0.00 & 0.04 & 2.95 & 0.00 & 0.56 & 0.09 \\
2.0 & 22.96 & 0.00 & 0.03 & 2.64 & 0.00 & 0.31 & 0.06 \\
2.5 & 22.95 & 0.00 & 0.03 & 2.51 & 0.00 & 0.25 & 0.05 \\
3.0 & 22.94 & 0.00 & 0.03 & 2.42 & 0.00 & 0.20 & 0.05 \\
3.5 & 22.96 & 0.20 & 0.03 & 2.80 & 0.02 & 0.37 & 0.08 \\
4.0 & 23.40 & 0.63 & 0.08 & 4.59 & 0.07 & 1.08 & 0.36 \\
4.5 & 23.54 & 0.38 & 0.10 & 4.80 & 0.04 & 0.98 & 0.38 \\
5.0 & 23.51 & 0.31 & 0.10 & 4.88 & 0.03 & 1.18 & 0.32 \\
5.1 & 23.92 & 0.87 & 0.11 & 6.25 & 0.10 & 1.41 & 0.55 \\
5.2 & 24.09 & 0.77 & 0.14 & 6.92 & 0.08 & 0.68 & 0.77 \\
5.3 & 23.84 & 0.63 & 0.13 & 6.29 & 0.07 & 0.50 & 0.72 \\
5.4 & 23.85 & 0.48 & 0.13 & 6.01 & 0.05 & 0.32 & 0.83 \\
\hline
\end{tabular}

Table 15.c. Emission line spectrum at solar metallicity (II)

\begin{tabular}{cccccccc}
\hline \hline Age & {$[\mathrm{NII}] 6584$} & {$[\mathrm{SII}] 6716$} & [SII] 6731 & [SIII] 9532 & [CII] 158 $\mu \mathrm{m}$ & $\mathrm{EW}(\mathrm{H} \beta)$ & $T_{e}(K)$ \\
\hline 1.5 & 1.34 & 0.70 & 0.48 & 0.78 & 1.76 & 197.21 & 6840 \\
2.0 & 1.31 & 0.69 & 0.48 & 0.67 & 1.94 & 169.94 & 6680 \\
2.5 & 1.28 & 0.68 & 0.47 & 0.64 & 1.99 & 162.93 & 6610 \\
3.0 & 1.28 & 0.71 & 0.49 & 0.58 & 2.09 & 137.09 & 6570 \\
3.5 & 1.42 & 0.90 & 0.62 & 0.54 & 2.45 & 115.13 & 6740 \\
4.0 & 1.98 & 1.46 & 1.01 & 0.77 & 3.09 & 76.12 & 7400 \\
4.5 & 2.02 & 1.46 & 1.01 & 0.77 & 2.76 & 95.52 & 7460 \\
5.0 & 1.95 & 1.27 & 0.88 & 0.92 & 2.22 & 121.96 & 7580 \\
5.1 & 2.46 & 1.86 & 1.29 & 0.90 & 3.38 & 102.36 & 7810 \\
5.2 & 2.81 & 2.39 & 1.65 & 0.67 & 4.22 & 54.81 & 7820 \\
5.3 & 2.64 & 2.31 & 1.59 & 0.58 & 4.25 & 48.32 & 7680 \\
5.4 & 2.63 & 2.49 & 1.72 & 0.47 & 4.97 & 37.28 & 7510 \\
\hline
\end{tabular}

Table 15.d. Emission line spectrum at twice solar metallicity (I)

\begin{tabular}{cccccccc}
\hline \hline Age & Ly $\alpha 1216$ & HeII 1640 & MgII 2800 & [OII] 3727 & HeII 4686 & [OIII] 5007 & [OI] 6300 \\
\hline 1.5 & 22.62 & 0.00 & 0.01 & 1.10 & 0.00 & 0.14 & 0.06 \\
2.0 & 22.62 & 0.00 & 0.01 & 0.85 & 0.00 & 0.08 & 0.04 \\
2.5 & 22.62 & 0.00 & 0.01 & 0.77 & 0.00 & 0.07 & 0.03 \\
3.0 & 22.61 & 0.00 & 0.01 & 0.72 & 0.00 & 0.05 & 0.03 \\
3.5 & 22.64 & 0.17 & 0.01 & 0.91 & 0.02 & 0.10 & 0.05 \\
4.0 & 22.75 & 0.58 & 0.03 & 2.21 & 0.06 & 0.39 & 0.28 \\
4.5 & 22.76 & 0.35 & 0.04 & 2.40 & 0.04 & 0.35 & 0.31 \\
5.0 & 22.76 & 0.29 & 0.04 & 2.52 & 0.03 & 0.41 & 0.27 \\
5.1 & 22.89 & 0.82 & 0.05 & 3.64 & 0.09 & 0.62 & 0.49 \\
5.2 & 22.92 & 0.71 & 0.06 & 3.99 & 0.08 & 0.36 & 0.66 \\
5.3 & 22.88 & 0.59 & 0.05 & 3.40 & 0.06 & 0.26 & 0.59 \\
5.4 & 22.85 & 0.44 & 0.05 & 3.13 & 0.05 & 0.17 & 0.64 \\
\hline
\end{tabular}

Table 15.e. Emission line spectrum at twice solar metallicity (II)

\begin{tabular}{cccccccc}
\hline \hline Age & {$[\mathrm{NII}] 6584$} & {$[\mathrm{SII}] 6716$} & [SII] 6731 & [SIII] 9532 & [CII] 158 $\mu \mathrm{m}$ & $\mathrm{EW}(\mathrm{H} \beta)$ & $T_{e}(K)$ \\
\hline 1.5 & 0.90 & 0.52 & 0.36 & 0.57 & 3.05 & 200.18 & 5090 \\
2.0 & 0.82 & 0.48 & 0.33 & 0.49 & 3.32 & 171.80 & 5000 \\
2.5 & 0.79 & 0.46 & 0.32 & 0.46 & 3.38 & 166.03 & 4930 \\
3.0 & 0.77 & 0.48 & 0.33 & 0.42 & 3.55 & 139.04 & 4900 \\
3.5 & 0.89 & 0.61 & 0.42 & 0.39 & 3.99 & 115.36 & 5010 \\
4.0 & 1.59 & 1.24 & 0.85 & 0.65 & 4.95 & 74.58 & 5700 \\
4.5 & 1.65 & 1.28 & 0.88 & 0.65 & 4.51 & 94.64 & 5790 \\
5.0 & 1.62 & 1.15 & 0.79 & 0.76 & 3.85 & 120.20 & 5810 \\
5.1 & 2.27 & 1.79 & 1.23 & 0.85 & 5.50 & 98.76 & 6180 \\
5.2 & 2.62 & 2.32 & 1.59 & 0.68 & 6.93 & 53.10 & 6270 \\
5.3 & 2.37 & 2.16 & 1.48 & 0.58 & 7.01 & 46.54 & 6110 \\
5.4 & 2.30 & 2.24 & 1.54 & 0.48 & 7.83 & 35.86 & 6010 \\
\hline
\end{tabular}


TABLE 16

$M_{T}=6 \times 10^{5} M_{\odot}$, IMF PARAMETERS: $\alpha=3.35, m_{\mathrm{low}}=0.85 M_{\odot}, m_{\mathrm{up}}=120 M_{\odot}$

Table 16.a. Parameters independent of metallicity

\begin{tabular}{ccccccccc}
\hline \hline Age & $\log L_{L y_{\alpha}}$ & $\log Q H$ & $\mathrm{QHe} / \mathrm{QH}$ & $\mathrm{QO} / \mathrm{QH}$ & $\log u$ & $\log I_{H}$ & $\epsilon$ & $M_{H I I} / \mathrm{M}_{\odot}$ \\
\hline 1.5 & 40.86 & 51.34 & 0.21 & 0.05 & -2.99 & 39.05 & 0.033 & $0.53 \mathrm{E}+06$ \\
2.0 & 40.79 & 51.30 & 0.15 & 0.03 & -3.04 & 39.00 & 0.030 & $0.46 \mathrm{E}+06$ \\
2.5 & 40.79 & 51.30 & 0.13 & 0.02 & -3.03 & 39.00 & 0.030 & $0.49 \mathrm{E}+06$ \\
3.0 & 40.79 & 51.29 & 0.15 & 0.03 & -3.05 & 38.99 & 0.030 & $0.45 \mathrm{E}+06$ \\
3.5 & 40.88 & 51.26 & 0.27 & 0.17 & -3.08 & 38.98 & 0.027 & $0.43 \mathrm{E}+06$ \\
4.0 & 40.76 & 51.12 & 0.28 & 0.19 & -3.22 & 38.85 & 0.020 & $0.31 \mathrm{E}+06$ \\
4.5 & 40.67 & 51.06 & 0.28 & 0.17 & -3.27 & 38.78 & 0.018 & $0.28 \mathrm{E}+06$ \\
5.0 & 40.76 & 51.15 & 0.33 & 0.16 & -3.18 & 38.87 & 0.022 & $0.34 \mathrm{E}+06$ \\
5.1 & 40.74 & 51.04 & 0.38 & 0.26 & -3.29 & 38.78 & 0.017 & $0.27 \mathrm{E}+06$ \\
5.2 & 40.48 & 50.79 & 0.38 & 0.27 & -3.55 & 38.52 & 0.009 & $0.14 \mathrm{E}+06$ \\
5.3 & 40.42 & 50.74 & 0.33 & 0.24 & -3.60 & 38.47 & 0.008 & $0.13 \mathrm{E}+06$ \\
5.4 & 40.31 & 50.63 & 0.32 & 0.23 & -3.70 & 38.37 & 0.007 & $0.10 \mathrm{E}+06$ \\
\hline
\end{tabular}

Table 16.b. Emission line spectrum at solar metallicity (I)

\begin{tabular}{cccccccc}
\hline \hline Age & Ly $\alpha$ 1216 & HeII 1640 & MgII 2800 & [OII] 3727 & HeII 4686 & [OIII] 5007 & [OI] 6300 \\
\hline 1.5 & 22.95 & 0.00 & 0.03 & 2.37 & 0.00 & 0.79 & 0.06 \\
2.0 & 22.92 & 0.00 & 0.02 & 2.21 & 0.00 & 0.44 & 0.04 \\
2.5 & 22.91 & 0.00 & 0.02 & 2.10 & 0.00 & 0.34 & 0.03 \\
3.0 & 22.93 & 0.00 & 0.02 & 2.11 & 0.00 & 0.48 & 0.04 \\
3.5 & 23.30 & 0.62 & 0.06 & 3.30 & 0.07 & 1.73 & 0.22 \\
4.0 & 23.37 & 0.73 & 0.07 & 3.98 & 0.08 & 1.57 & 0.28 \\
4.5 & 23.46 & 0.40 & 0.08 & 4.14 & 0.04 & 1.31 & 0.29 \\
5.0 & 23.47 & 0.34 & 0.09 & 4.23 & 0.04 & 1.59 & 0.25 \\
5.1 & 23.84 & 0.98 & 0.10 & 5.47 & 0.11 & 2.07 & 0.44 \\
5.2 & 24.04 & 0.91 & 0.12 & 6.63 & 0.10 & 1.20 & 0.63 \\
5.3 & 23.85 & 0.77 & 0.11 & 6.11 & 0.08 & 0.92 & 0.59 \\
5.4 & 23.89 & 0.59 & 0.12 & 6.09 & 0.06 & 0.62 & 0.71 \\
\hline
\end{tabular}

Table 16.c. Emission line spectrum at solar metallicity (II)

\begin{tabular}{cccccccc}
\hline \hline Age & {$[\mathrm{NII}] 6584$} & [SII] 6716 & [SII] 6731 & [SIII] 9532 & [CII] $158 \mu \mathrm{m}$ & $\left.\mathrm{EW}_{(\mathrm{H}}\right)$ & $T_{e}(K)$ \\
\hline 1.5 & 1.04 & 0.50 & 0.34 & 0.82 & 1.39 & 218.65 & 6630 \\
2.0 & 1.08 & 0.50 & 0.35 & 0.73 & 1.65 & 182.83 & 6510 \\
2.5 & 1.08 & 0.50 & 0.34 & 0.70 & 1.76 & 172.30 & 6440 \\
3.0 & 1.05 & 0.51 & 0.35 & 0.70 & 1.66 & 161.06 & 6450 \\
3.5 & 1.40 & 0.93 & 0.64 & 0.94 & 2.16 & 146.64 & 7190 \\
4.0 & 1.69 & 1.18 & 0.82 & 0.90 & 2.59 & 80.08 & 7360 \\
4.5 & 1.73 & 1.18 & 0.81 & 0.85 & 2.31 & 94.25 & 7340 \\
5.0 & 1.63 & 1.01 & 0.70 & 1.00 & 1.79 & 123.65 & 7470 \\
5.1 & 2.12 & 1.53 & 1.06 & 1.04 & 2.85 & 103.06 & 7780 \\
5.2 & 2.61 & 2.07 & 1.43 & 0.82 & 3.62 & 55.68 & 7830 \\
5.3 & 2.50 & 2.02 & 1.40 & 0.72 & 3.67 & 48.57 & 7710 \\
5.4 & 2.58 & 2.25 & 1.55 & 0.61 & 4.37 & 37.94 & 7590 \\
\hline
\end{tabular}

Table 16.d. Emission line spectrum at twice solar metallicity (I)

\begin{tabular}{cccccccc}
\hline \hline Age & Ly $\alpha 1216$ & HeII 1640 & MgII 2800 & [OII] 3727 & HeII 4686 & [OIII] 5007 & [OI] 6300 \\
\hline 1.5 & 22.47 & 0.00 & 0.01 & 0.91 & 0.00 & 0.16 & 0.05 \\
2.0 & 22.52 & 0.00 & 0.01 & 0.71 & 0.00 & 0.08 & 0.03 \\
2.5 & 22.53 & 0.00 & 0.00 & 0.62 & 0.00 & 0.06 & 0.02 \\
3.0 & 22.49 & 0.00 & 0.01 & 0.67 & 0.00 & 0.08 & 0.03 \\
3.5 & 22.58 & 0.57 & 0.02 & 1.63 & 0.06 & 0.41 & 0.19 \\
4.0 & 22.70 & 0.68 & 0.03 & 1.97 & 0.07 & 0.45 & 0.24 \\
4.5 & 22.69 & 0.36 & 0.03 & 2.11 & 0.04 & 0.38 & 0.25 \\
5.0 & 22.67 & 0.32 & 0.04 & 2.26 & 0.04 & 0.47 & 0.23 \\
5.1 & 22.85 & 0.94 & 0.05 & 3.31 & 0.10 & 0.77 & 0.41 \\
5.2 & 22.91 & 0.84 & 0.06 & 3.82 & 0.09 & 0.56 & 0.55 \\
5.3 & 22.87 & 0.71 & 0.05 & 3.34 & 0.08 & 0.43 & 0.50 \\
5.4 & 22.87 & 0.54 & 0.05 & 3.34 & 0.06 & 0.31 & 0.58 \\
\hline
\end{tabular}

Table 16.e. Emission line spectrum at twice solar metallicity (II)

\begin{tabular}{cccccccc}
\hline \hline Age & {$[\mathrm{NII}] 6584$} & [SII] 6716 & [SII] 6731 & [SIII] 9532 & [CII] 158 $\mu \mathrm{m}$ & $\mathrm{EW}(\mathrm{H} \beta)$ & $T_{e}(K)$ \\
\hline 1.5 & 0.68 & 0.36 & 0.25 & 0.50 & 2.33 & 221.94 & 4720 \\
2.0 & 0.65 & 0.34 & 0.23 & 0.46 & 2.77 & 186.49 & 4720 \\
2.5 & 0.62 & 0.33 & 0.23 & 0.44 & 2.93 & 177.01 & 4690 \\
3.0 & 0.61 & 0.34 & 0.23 & 0.41 & 2.75 & 164.41 & 4620 \\
3.5 & 1.07 & 0.76 & 0.52 & 0.60 & 3.44 & 146.29 & 5240 \\
4.0 & 1.34 & 1.00 & 0.69 & 0.66 & 4.15 & 78.22 & 5520 \\
4.5 & 1.38 & 1.02 & 0.70 & 0.63 & 3.77 & 93.03 & 5560 \\
5.0 & 1.35 & 0.91 & 0.63 & 0.73 & 3.08 & 122.31 & 5590 \\
5.1 & 1.96 & 1.47 & 1.01 & 0.90 & 4.66 & 99.30 & 6040 \\
5.2 & 2.41 & 1.98 & 1.36 & 0.79 & 5.84 & 54.44 & 6250 \\
5.3 & 2.23 & 1.88 & 1.29 & 0.68 & 6.01 & 47.86 & 6100 \\
5.4 & 2.31 & 2.07 & 1.43 & 0.60 & 6.89 & 36.75 & 6060 \\
\hline
\end{tabular}


TABLE 17

$M_{T}=8 \times 10^{5} M_{\odot}$, IMF PARAMETERS: $\alpha=3.35, m_{\mathrm{low}}=0.85 M_{\odot}, m_{\mathrm{up}}=120 M_{\odot}$

Table 17.a. Parameters independent of metallicity

\begin{tabular}{ccccccccc}
\hline \hline Age & $\log L_{L y_{\alpha}}$ & $\log Q H$ & $Q_{\mathrm{He} / Q \mathrm{H}}$ & $\mathrm{QO} / \mathrm{QH}$ & $\log u$ & $\log I_{H_{\beta}}$ & $\epsilon$ & $M_{H I I} / \mathrm{M}_{\odot}$ \\
\hline 1.5 & 40.98 & 51.47 & 0.21 & 0.05 & -2.87 & 39.17 & 0.044 & $0.69 \mathrm{E}+06$ \\
2.0 & 40.92 & 51.42 & 0.15 & 0.03 & -2.91 & 39.13 & 0.040 & $0.64 \mathrm{E}+06$ \\
2.5 & 40.91 & 51.43 & 0.13 & 0.02 & -2.91 & 39.12 & 0.040 & $0.64 \mathrm{E}+06$ \\
3.0 & 40.90 & 51.41 & 0.14 & 0.03 & -2.93 & 39.11 & 0.039 & $0.60 \mathrm{E}+06$ \\
3.5 & 40.99 & 51.37 & 0.25 & 0.16 & -2.96 & 39.09 & 0.036 & $0.57 \mathrm{E}+06$ \\
4.0 & 40.90 & 51.25 & 0.28 & 0.19 & -3.09 & 38.98 & 0.027 & $0.41 \mathrm{E}+06$ \\
4.5 & 40.81 & 51.20 & 0.29 & 0.18 & -3.14 & 38.91 & 0.024 & $0.37 \mathrm{E}+06$ \\
5.0 & 40.88 & 51.27 & 0.33 & 0.16 & -3.06 & 38.98 & 0.028 & $0.46 \mathrm{E}+06$ \\
5.1 & 40.86 & 51.17 & 0.37 & 0.26 & -3.17 & 38.90 & 0.022 & $0.35 \mathrm{E}+06$ \\
5.2 & 40.60 & 50.91 & 0.37 & 0.26 & -3.43 & 38.64 & 0.012 & $0.19 \mathrm{E}+06$ \\
5.3 & 40.53 & 50.86 & 0.33 & 0.23 & -3.47 & 38.59 & 0.011 & $0.18 \mathrm{E}+06$ \\
5.4 & 40.43 & 50.76 & 0.31 & 0.23 & -3.58 & 38.49 & 0.009 & $0.14 \mathrm{E}+06$ \\
\hline
\end{tabular}

Table 17.b. Emission line spectrum at solar metallicity (I)

\begin{tabular}{cccccccc}
\hline \hline Age & Ly $\alpha 1216$ & HeII 1640 & MgII 2800 & [OII] 3727 & HeII 4686 & [OIII] 5007 & [OI] 6300 \\
\hline 1.5 & 22.92 & 0.00 & 0.03 & 2.02 & 0.00 & 0.84 & 0.05 \\
2.0 & 22.90 & 0.00 & 0.02 & 1.96 & 0.00 & 0.52 & 0.03 \\
2.5 & 22.90 & 0.00 & 0.02 & 1.90 & 0.00 & 0.41 & 0.03 \\
3.0 & 22.90 & 0.00 & 0.02 & 1.85 & 0.00 & 0.50 & 0.03 \\
3.5 & 23.18 & 0.61 & 0.05 & 2.67 & 0.07 & 1.72 & 0.16 \\
4.0 & 23.34 & 0.79 & 0.06 & 3.46 & 0.09 & 1.91 & 0.24 \\
4.5 & 23.45 & 0.44 & 0.08 & 3.71 & 0.05 & 1.62 & 0.25 \\
5.0 & 23.43 & 0.36 & 0.08 & 3.76 & 0.04 & 1.82 & 0.21 \\
5.1 & 23.76 & 1.02 & 0.09 & 4.83 & 0.11 & 2.46 & 0.36 \\
5.2 & 23.93 & 0.96 & 0.11 & 6.05 & 0.10 & 1.55 & 0.52 \\
5.3 & 23.74 & 0.82 & 0.10 & 5.56 & 0.09 & 1.22 & 0.49 \\
5.4 & 23.83 & 0.65 & 0.11 & 5.79 & 0.07 & 0.86 & 0.62 \\
\hline
\end{tabular}

Table 17.c. Emission line spectrum at solar metallicity (II)

\begin{tabular}{cccccccc}
\hline \hline Age & [NII] 6584 & [SII] 6716 & [SII] 6731 & [SIII] 9532 & [CII] 158 $\mu \mathrm{m}$ & $\mathrm{EW}(\mathrm{H} \beta)$ & $T_{e}(K)$ \\
\hline 1.5 & 0.87 & 0.40 & 0.28 & 0.80 & 1.21 & 217.67 & 6460 \\
2.0 & 0.95 & 0.41 & 0.28 & 0.75 & 1.49 & 182.27 & 6380 \\
2.5 & 0.96 & 0.42 & 0.29 & 0.72 & 1.61 & 172.14 & 6340 \\
3.0 & 0.92 & 0.42 & 0.29 & 0.71 & 1.52 & 157.56 & 6310 \\
3.5 & 1.16 & 0.74 & 0.51 & 0.93 & 1.86 & 142.00 & 7000 \\
4.0 & 1.46 & 1.00 & 0.69 & 0.97 & 2.27 & 78.50 & 7310 \\
4.5 & 1.52 & 1.01 & 0.69 & 0.92 & 2.03 & 95.90 & 7270 \\
5.0 & 1.42 & 0.87 & 0.60 & 1.03 & 1.54 & 120.74 & 7370 \\
5.1 & 1.85 & 1.31 & 0.90 & 1.12 & 2.46 & 101.22 & 7740 \\
5.2 & 2.37 & 1.81 & 1.25 & 0.90 & 3.19 & 54.76 & 7800 \\
5.3 & 2.28 & 1.76 & 1.22 & 0.79 & 3.27 & 47.95 & 7650 \\
5.4 & 2.43 & 2.02 & 1.40 & 0.68 & 3.95 & 37.12 & 7580 \\
\hline
\end{tabular}

Table 17.d. Emission line spectrum at twice solar metallicity (I)

\begin{tabular}{cccccccc}
\hline \hline Age & Ly $\alpha$ 1216 & HeII 1640 & MgII 2800 & [OII] 3727 & HeII 4686 & [OIII] 5007 & [OI] 6300 \\
\hline 1.5 & 22.37 & 0.00 & 0.01 & 0.79 & 0.00 & 0.16 & 0.04 \\
2.0 & 22.44 & 0.00 & 0.00 & 0.63 & 0.00 & 0.08 & 0.02 \\
2.5 & 22.46 & 0.00 & 0.00 & 0.56 & 0.00 & 0.06 & 0.02 \\
3.0 & 22.41 & 0.00 & 0.00 & 0.59 & 0.00 & 0.08 & 0.02 \\
3.5 & 22.49 & 0.56 & 0.02 & 1.29 & 0.06 & 0.21 & 0.14 \\
4.0 & 22.62 & 0.74 & 0.02 & 1.76 & 0.08 & 0.47 & 0.21 \\
4.5 & 22.61 & 0.41 & 0.03 & 1.97 & 0.05 & 0.41 & 0.23 \\
5.0 & 22.59 & 0.33 & 0.03 & 2.06 & 0.04 & 0.48 & 0.20 \\
5.1 & 22.79 & 0.97 & 0.04 & 2.87 & 0.11 & 0.80 & 0.34 \\
5.2 & 22.88 & 0.89 & 0.05 & 3.44 & 0.10 & 0.64 & 0.46 \\
5.3 & 22.84 & 0.75 & 0.04 & 3.00 & 0.08 & 0.49 & 0.42 \\
5.4 & 22.85 & 0.59 & 0.05 & 3.07 & 0.06 & 0.38 & 0.49 \\
\hline
\end{tabular}

Table 17.e. Emission line spectrum at twice solar metallicity (II)

\begin{tabular}{cccccccc}
\hline \hline Age & [NII] 6584 & [SII] 6716 & [SII] 6731 & [SIII] 9532 & [CII] $158 \mu \mathrm{m}$ & $\mathrm{EW}(\mathrm{H} \beta)$ & $T_{e}(K)$ \\
\hline 1.5 & 0.57 & 0.29 & 0.20 & 0.45 & 2.01 & 222.57 & 4500 \\
2.0 & 0.56 & 0.27 & 0.19 & 0.43 & 2.47 & 187.29 & 4520 \\
2.5 & 0.54 & 0.27 & 0.18 & 0.41 & 2.65 & 177.39 & 4520 \\
3.0 & 0.53 & 0.27 & 0.19 & 0.38 & 2.50 & 161.19 & 4430 \\
3.5 & 0.86 & 0.59 & 0.40 & 0.52 & 2.93 & 141.34 & 4950 \\
4.0 & 1.15 & 0.83 & 0.57 & 0.64 & 3.63 & 77.06 & 5350 \\
4.5 & 1.22 & 0.87 & 0.60 & 0.61 & 3.25 & 95.18 & 5400 \\
5.0 & 1.18 & 0.77 & 0.53 & 0.68 & 2.66 & 120.02 & 5400 \\
5.1 & 1.66 & 1.22 & 0.84 & 0.87 & 4.02 & 98.86 & 5880 \\
5.2 & 2.14 & 1.70 & 1.17 & 0.81 & 5.19 & 53.43 & 6130 \\
5.3 & 1.98 & 1.61 & 1.11 & 0.71 & 5.29 & 47.15 & 6000 \\
5.4 & 2.10 & 1.82 & 1.25 & 0.63 & 6.16 & 36.53 & 6000 \\
\hline
\end{tabular}


TABLE 18

$$
M_{T}=2 \times 10^{6} M_{\odot} \text {, IMF PARAMETERS: } \alpha=3.35, m_{\mathrm{low}}=0.85 M_{\odot}, m_{\mathrm{up}}=120 M_{\odot}
$$

Table 18.a. Parameters independent of metallicity

\begin{tabular}{ccccccccc}
\hline \hline Age & $\log L_{L y_{\alpha}}$ & $\log Q H$ & QHe/QH & QO/QH & $\log u$ & $\log I_{H}$ & $\epsilon$ & $M_{H I I} / \mathrm{M}_{\odot}$ \\
\hline 1.5 & 41.42 & 51.90 & 0.21 & 0.05 & -2.43 & 39.61 & 0.121 & $0.20 \mathrm{E}+07$ \\
2.0 & 41.35 & 51.86 & 0.15 & 0.03 & -2.48 & 39.56 & 0.110 & $0.17 \mathrm{E}+07$ \\
2.5 & 41.35 & 51.86 & 0.13 & 0.02 & -2.48 & 39.56 & 0.110 & $0.17 \mathrm{E}+07$ \\
3.0 & 41.34 & 51.84 & 0.15 & 0.03 & -2.49 & 39.54 & 0.105 & $0.17 \mathrm{E}+07$ \\
3.5 & 41.44 & 51.81 & 0.28 & 0.18 & -2.52 & 39.52 & 0.099 & $0.16 \mathrm{E}+07$ \\
4.0 & 41.34 & 51.68 & 0.31 & 0.21 & -2.66 & 39.40 & 0.072 & $0.11 \mathrm{E}+07$ \\
4.5 & 41.23 & 51.61 & 0.30 & 0.19 & -2.73 & 39.33 & 0.062 & $0.94 \mathrm{E}+06$ \\
5.0 & 41.29 & 51.68 & 0.33 & 0.17 & -2.66 & 39.39 & 0.073 & $0.11 \mathrm{E}+07$ \\
5.1 & 41.27 & 51.57 & 0.38 & 0.26 & -2.77 & 39.30 & 0.057 & $0.85 \mathrm{E}+06$ \\
5.2 & 41.01 & 51.32 & 0.37 & 0.27 & -3.02 & 39.05 & 0.031 & $0.49 \mathrm{E}+06$ \\
5.3 & 40.94 & 51.27 & 0.33 & 0.24 & -3.07 & 39.00 & 0.028 & $0.43 \mathrm{E}+06$ \\
5.4 & 40.84 & 51.16 & 0.31 & 0.23 & -3.18 & 38.90 & 0.022 & $0.33 \mathrm{E}+06$ \\
\hline
\end{tabular}

Table 18.b. Emission line spectrum at solar metallicity (I)

\begin{tabular}{cccccccc}
\hline \hline Age & Ly $\alpha 1216$ & HeII 1640 & MgII 2800 & [OII] 3727 & HeII 4686 & [OIII] 5007 & [OI] 6300 \\
\hline 1.5 & 22.78 & 0.00 & 0.02 & 1.12 & 0.00 & 0.95 & 0.03 \\
2.0 & 22.77 & 0.00 & 0.01 & 1.20 & 0.00 & 0.58 & 0.01 \\
2.5 & 22.78 & 0.00 & 0.01 & 1.27 & 0.00 & 0.44 & 0.01 \\
3.0 & 22.76 & 0.00 & 0.01 & 1.10 & 0.00 & 0.55 & 0.01 \\
3.5 & 23.23 & 0.74 & 0.04 & 1.69 & 0.08 & 2.63 & 0.09 \\
4.0 & 23.34 & 0.99 & 0.05 & 2.15 & 0.11 & 3.10 & 0.14 \\
4.5 & 23.39 & 0.52 & 0.06 & 2.46 & 0.06 & 2.40 & 0.14 \\
5.0 & 23.35 & 0.39 & 0.06 & 2.44 & 0.04 & 2.47 & 0.12 \\
5.1 & 23.62 & 1.18 & 0.07 & 3.07 & 0.13 & 3.79 & 0.20 \\
5.2 & 23.70 & 1.16 & 0.08 & 4.01 & 0.13 & 2.89 & 0.29 \\
5.3 & 23.55 & 1.01 & 0.07 & 3.77 & 0.11 & 2.36 & 0.27 \\
5.4 & 23.67 & 0.86 & 0.08 & 4.31 & 0.09 & 2.06 & 0.37 \\
\hline
\end{tabular}

Table 18.c. Emission line spectrum at solar metallicity (II)

\begin{tabular}{cccccccc}
\hline \hline Age & {$[\mathrm{NII}] 6584$} & {$[\mathrm{SII}] 6716$} & {$[\mathrm{SII}] 6731$} & [SIII] 9532 & [CII] $158 \mu \mathrm{m}$ & $\mathrm{EW}(\mathrm{H} \beta)$ & $T_{e}(K)$ \\
\hline 1.5 & 0.44 & 0.19 & 0.13 & 0.69 & 0.68 & 232.50 & 5860 \\
2.0 & 0.55 & 0.19 & 0.13 & 0.68 & 0.98 & 193.45 & 5820 \\
2.5 & 0.62 & 0.20 & 0.14 & 0.68 & 1.16 & 181.26 & 5850 \\
3.0 & 0.52 & 0.19 & 0.13 & 0.62 & 0.99 & 165.85 & 5700 \\
3.5 & 0.64 & 0.40 & 0.27 & 1.07 & 0.97 & 148.44 & 6900 \\
4.0 & 0.83 & 0.55 & 0.38 & 1.19 & 1.30 & 80.00 & 7230 \\
4.5 & 0.90 & 0.57 & 0.39 & 1.04 & 1.15 & 98.37 & 7050 \\
5.0 & 0.83 & 0.49 & 0.34 & 1.08 & 0.83 & 122.64 & 7060 \\
5.1 & 1.09 & 0.74 & 0.51 & 1.34 & 1.41 & 100.71 & 7660 \\
5.2 & 1.51 & 1.07 & 0.74 & 1.16 & 2.00 & 55.85 & 7680 \\
5.3 & 1.50 & 1.06 & 0.73 & 1.04 & 2.10 & 49.00 & 7510 \\
5.4 & 1.76 & 1.32 & 0.91 & 0.97 & 2.71 & 38.17 & 7530 \\
\hline
\end{tabular}

Table 18.d. Emission line spectrum at twice solar metallicity (I)

\begin{tabular}{cccccccc}
\hline Age & Ly $\alpha 1216$ & HeII 1640 & MgII 2800 & [OII] 3727 & HeII 4686 & [OIII] 5007 & [OI] 6300 \\
\hline 1.5 & 22.00 & 0.00 & 0.00 & 0.45 & 0.00 & 0.14 & 0.02 \\
2.0 & 22.11 & 0.00 & 0.00 & 0.39 & 0.00 & 0.06 & 0.01 \\
2.5 & 22.17 & 0.00 & 0.00 & 0.38 & 0.00 & 0.04 & 0.01 \\
3.0 & 22.05 & 0.00 & 0.00 & 0.36 & 0.00 & 0.05 & 0.01 \\
3.5 & 22.26 & 0.69 & 0.02 & 0.93 & 0.08 & 0.35 & 0.09 \\
4.0 & 22.36 & 0.95 & 0.02 & 1.30 & 0.11 & 0.51 & 0.14 \\
4.5 & 22.37 & 0.49 & 0.03 & 1.49 & 0.05 & 0.47 & 0.14 \\
5.0 & 22.34 & 0.37 & 0.03 & 1.44 & 0.04 & 0.54 & 0.12 \\
5.1 & 21.86 & 2.43 & 0.00 & 0.00 & 0.27 & 0.01 & 0.00 \\
5.2 & 22.72 & 1.11 & 0.04 & 2.60 & 0.12 & 0.85 & 0.29 \\
5.3 & 22.66 & 0.95 & 0.03 & 2.25 & 0.11 & 0.61 & 0.26 \\
5.4 & 22.75 & 0.80 & 0.04 & 2.51 & 0.09 & 0.60 & 0.34 \\
\hline
\end{tabular}

Table 18.e. Emission line spectrum at twice solar metallicity (II)

\begin{tabular}{cccccccc}
\hline \hline Age & [NII] 6584 & [SII] 6716 & [SII] 6731 & [SIII] 9532 & [CII] 158 $\mu \mathrm{m}$ & $\mathrm{EW}\left(\mathrm{H}_{\beta}\right)$ & $T_{e}(K)$ \\
\hline 1.5 & 0.29 & 0.13 & 0.09 & 0.28 & 1.04 & 241.96 & 3680 \\
2.0 & 0.32 & 0.12 & 0.08 & 0.30 & 1.54 & 200.90 & 3810 \\
2.5 & 0.34 & 0.12 & 0.08 & 0.31 & 1.82 & 188.29 & 3900 \\
3.0 & 0.30 & 0.12 & 0.08 & 0.26 & 1.53 & 171.01 & 3680 \\
3.5 & 0.50 & 0.32 & 0.22 & 0.39 & 1.60 & 153.62 & 4310 \\
4.0 & 0.71 & 0.47 & 0.32 & 0.54 & 2.15 & 79.15 & 4810 \\
4.5 & 0.77 & 0.50 & 0.34 & 0.49 & 1.85 & 98.37 & 4800 \\
5.0 & 0.71 & 0.43 & 0.30 & 0.52 & 1.41 & 123.90 & 4760 \\
5.1 & 0.00 & 0.00 & 0.00 & 0.04 & 0.53 & 12.72 & 2460 \\
5.2 & 1.42 & 1.02 & 0.70 & 0.81 & 3.32 & 53.96 & 5680 \\
5.3 & 1.30 & 0.96 & 0.66 & 0.69 & 3.42 & 47.59 & 5540 \\
5.4 & 1.53 & 1.18 & 0.81 & 0.71 & 4.34 & 36.75 & 5670 \\
\hline
\end{tabular}


TABLE 19

$M_{T}=2 \times 10^{6} M_{\odot}$, IMF PARAMETERS: $\alpha=3.35, m_{\text {low }}=0.85 M_{\odot}, m_{\mathrm{up}}=60 M_{\odot}$

Table 19.a. Parameters independent of metallicity

\begin{tabular}{ccccccccc}
\hline \hline Age & $\log L_{L y_{\alpha}}$ & $\log Q H$ & $\mathrm{QHe} / \mathrm{QH}$ & $\mathrm{QO} / \mathrm{QH}$ & $\log u$ & $\log I_{H_{\beta}}$ & $\epsilon$ & $M_{H I I} / \mathrm{M}_{\odot}$ \\
\hline 1.5 & 41.24 & 51.73 & 0.19 & 0.04 & -2.60 & 39.44 & 0.082 & $0.13 \mathrm{E}+07$ \\
2.0 & 41.19 & 51.70 & 0.14 & 0.03 & -2.64 & 39.40 & 0.075 & $0.12 \mathrm{E}+07$ \\
2.5 & 41.20 & 51.71 & 0.14 & 0.02 & -2.63 & 39.41 & 0.078 & $0.12 \mathrm{E}+07$ \\
3.0 & 41.17 & 51.70 & 0.11 & 0.02 & -2.64 & 39.39 & 0.075 & $0.12 \mathrm{E}+07$ \\
3.5 & 41.04 & 51.58 & 0.05 & 0.01 & -2.75 & 39.28 & 0.058 & $0.93 \mathrm{E}+06$ \\
4.0 & 41.04 & 51.54 & 0.12 & 0.05 & -2.80 & 39.24 & 0.053 & $0.80 \mathrm{E}+06$ \\
4.5 & 41.23 & 51.61 & 0.30 & 0.19 & -2.73 & 39.33 & 0.062 & $0.94 \mathrm{E}+06$ \\
5.0 & 41.29 & 51.68 & 0.33 & 0.16 & -2.65 & 39.39 & 0.073 & $0.12 \mathrm{E}+07$ \\
5.1 & 41.27 & 51.57 & 0.38 & 0.26 & -2.77 & 39.30 & 0.057 & $0.85 \mathrm{E}+06$ \\
5.2 & 41.01 & 51.32 & 0.37 & 0.27 & -3.02 & 39.05 & 0.031 & $0.49 \mathrm{E}+06$ \\
5.3 & 40.94 & 51.27 & 0.33 & 0.24 & -3.07 & 39.00 & 0.028 & $0.43 \mathrm{E}+06$ \\
5.4 & 40.84 & 51.16 & 0.31 & 0.23 & -3.18 & 38.90 & 0.022 & $0.33 \mathrm{E}+06$ \\
\hline
\end{tabular}

Table 19.b. Emission line spectrum at solar metallicity (I)

\begin{tabular}{cccccccc}
\hline \hline Age & Ly $\alpha$ 1216 & HeII 1640 & MgII 2800 & [OII] 3727 & HeII 4686 & [OIII] 5007 & [OI] 6300 \\
\hline 1.5 & 22.82 & 0.00 & 0.02 & 1.37 & 0.00 & 0.82 & 0.03 \\
2.0 & 22.83 & 0.00 & 0.01 & 1.42 & 0.00 & 0.55 & 0.02 \\
2.5 & 22.83 & 0.00 & 0.01 & 1.43 & 0.00 & 0.50 & 0.02 \\
3.0 & 22.83 & 0.00 & 0.01 & 1.48 & 0.00 & 0.34 & 0.02 \\
3.5 & 22.85 & 0.00 & 0.01 & 1.60 & 0.00 & 0.10 & 0.02 \\
4.0 & 22.84 & 0.08 & 0.01 & 1.45 & 0.01 & 0.50 & 0.02 \\
4.5 & 23.39 & 0.52 & 0.06 & 2.46 & 0.06 & 2.40 & 0.14 \\
5.0 & 23.35 & 0.39 & 0.06 & 2.43 & 0.04 & 2.46 & 0.12 \\
5.1 & 23.61 & 1.18 & 0.07 & 3.06 & 0.13 & 3.78 & 0.20 \\
5.2 & 23.70 & 1.16 & 0.08 & 3.99 & 0.13 & 2.87 & 0.29 \\
5.3 & 23.55 & 1.01 & 0.07 & 3.76 & 0.11 & 2.35 & 0.27 \\
5.4 & 23.67 & 0.86 & 0.08 & 4.31 & 0.09 & 2.06 & 0.37 \\
\hline
\end{tabular}

Table 19.c. Emission line spectrum at solar metallicity (II)

\begin{tabular}{cccccccc}
\hline \hline Age & {$[$ [II] 6584} & {$[$ SII] 6716} & [SII] 6731 & [SIII] 9532 & [CII] 158 $\mu \mathrm{m}$ & $\mathrm{EW}(\mathrm{H} \beta)$ & $T_{e}(K)$ \\
\hline 1.5 & 0.59 & 0.24 & 0.17 & 0.72 & 0.93 & 173.51 \\
2.0 & 0.68 & 0.26 & 0.18 & 0.70 & 1.20 & 149.21 & 6010 \\
2.5 & 0.69 & 0.25 & 0.17 & 0.70 & 1.23 & 148.38 \\
3.0 & 0.78 & 0.27 & 0.19 & 0.69 & 1.47 & 136.02 & 6030 \\
3.5 & 0.97 & 0.35 & 0.24 & 0.65 & 2.05 & 95.94 & 6130 \\
4.0 & 0.80 & 0.36 & 0.25 & 0.61 & 1.60 & 56.19 & 6040 \\
4.5 & 0.90 & 0.57 & 0.39 & 1.04 & 1.14 & 98.24 & 7050 \\
5.0 & 0.83 & 0.49 & 0.34 & 1.08 & 0.83 & 122.45 & 7050 \\
5.1 & 1.09 & 0.74 & 0.51 & 1.33 & 1.42 & 100.76 & 7650 \\
5.2 & 1.51 & 1.07 & 0.74 & 1.16 & 2.01 & 55.75 & 7670 \\
5.3 & 1.50 & 1.06 & 0.73 & 1.04 & 2.11 & 48.91 & 7500 \\
5.4 & 1.76 & 1.32 & 0.91 & 0.97 & 2.71 & 38.11 & 7530 \\
\hline
\end{tabular}

Table 19.d. Emission line spectrum at twice solar metallicity (I)

\begin{tabular}{cccccccc}
\hline \hline Age & Ly $\alpha$ 1216 & HeII 1640 & MgII 2800 & [OII] 3727 & HeII 4686 & [OIII] 5007 & [OI] 6300 \\
\hline 1.5 & 22.14 & 0.00 & 0.00 & 0.50 & 0.00 & 0.11 & 0.02 \\
2.0 & 22.24 & 0.00 & 0.00 & 0.44 & 0.00 & 0.06 & 0.01 \\
2.5 & 22.26 & 0.00 & 0.00 & 0.44 & 0.00 & 0.05 & 0.01 \\
3.0 & 22.31 & 0.00 & 0.00 & 0.41 & 0.00 & 0.03 & 0.01 \\
3.5 & 22.45 & 0.00 & 0.00 & 0.38 & 0.00 & 0.01 & 0.01 \\
4.0 & 22.24 & 0.07 & 0.00 & 0.45 & 0.01 & 0.05 & 0.02 \\
4.5 & 22.37 & 0.49 & 0.03 & 1.49 & 0.05 & 0.48 & 0.14 \\
5.0 & 22.33 & 0.37 & 0.03 & 1.44 & 0.04 & 0.54 & 0.12 \\
5.1 & 21.86 & 2.43 & 0.00 & 0.00 & 0.27 & 0.01 & 0.00 \\
5.2 & 22.71 & 1.11 & 0.04 & 2.60 & 0.12 & 0.85 & 0.29 \\
5.3 & 22.66 & 0.95 & 0.03 & 2.24 & 0.11 & 0.61 & 0.26 \\
5.4 & 22.75 & 0.80 & 0.04 & 2.51 & 0.09 & 0.61 & 0.34 \\
\hline
\end{tabular}

Table 19.e. Emission line spectrum at twice solar metallicity (II)

\begin{tabular}{cccccccc}
\hline \hline Age & [NII] 6584 & [SII] 6716 & [SII] 6731 & [SIII] 9532 & [CII] $158 \mu \mathrm{m}$ & $\mathrm{EW}(\mathrm{H} \beta)$ & $T_{e}(K)$ \\
\hline 1.5 & 0.36 & 0.16 & 0.11 & 0.32 & 1.45 & 178.11 & 3940 \\
2.0 & 0.38 & 0.16 & 0.11 & 0.33 & 1.91 & 153.93 & 4050 \\
2.5 & 0.39 & 0.16 & 0.11 & 0.34 & 1.98 & 152.99 & 4070 \\
3.0 & 0.41 & 0.17 & 0.11 & 0.35 & 2.36 & 140.92 & 4150 \\
3.5 & 0.44 & 0.20 & 0.14 & 0.35 & 3.24 & 98.07 & 4290 \\
4.0 & 0.42 & 0.21 & 0.15 & 0.28 & 2.47 & 57.57 & 4100 \\
4.5 & 0.77 & 0.50 & 0.34 & 0.49 & 1.86 & 98.22 & 4790 \\
5.0 & 0.71 & 0.43 & 0.30 & 0.52 & 1.41 & 123.99 & 4770 \\
5.1 & 0.00 & 0.00 & 0.00 & 0.04 & 0.53 & 12.73 & 2450 \\
5.2 & 1.41 & 1.02 & 0.70 & 0.81 & 3.31 & 53.86 & 5680 \\
5.3 & 1.30 & 0.96 & 0.66 & 0.68 & 3.42 & 47.51 & 5540 \\
5.4 & 1.53 & 1.18 & 0.81 & 0.71 & 4.34 & 36.69 & 5670 \\
\hline
\end{tabular}


TABLE 20

$M_{T}=2 \times 10^{6} M_{\odot}$, IMF PARAMETERS: $\alpha=3.35, m_{\text {low }}=0.85 M_{\odot}, m_{\mathrm{up}}=40 M_{\odot}$

Table 20.a. Parameters independent of metallicity

\begin{tabular}{ccccccccc}
\hline \hline Age & $\log L_{L y_{\alpha}}$ & $\log Q H$ & $\mathrm{QHe} / \mathrm{QH}$ & $\mathrm{QO} / \mathrm{QH}$ & $\log u$ & $\log I_{H}$ & $\epsilon$ & $M_{H I I} / \mathrm{M}_{\odot}$ \\
\hline 1.5 & 41.01 & 51.52 & 0.14 & 0.02 & -2.81 & 39.22 & 0.050 & $0.82 \mathrm{E}+06$ \\
2.0 & 40.97 & 51.49 & 0.11 & 0.02 & -2.85 & 39.18 & 0.046 & $0.73 \mathrm{E}+06$ \\
2.5 & 40.98 & 51.50 & 0.12 & 0.02 & -2.84 & 39.19 & 0.048 & $0.74 \mathrm{E}+06$ \\
3.0 & 40.97 & 51.49 & 0.10 & 0.02 & -2.85 & 39.18 & 0.047 & $0.72 \mathrm{E}+06$ \\
3.5 & 40.91 & 51.45 & 0.05 & 0.01 & -2.89 & 39.15 & 0.043 & $0.65 \mathrm{E}+06$ \\
4.0 & 40.96 & 51.50 & 0.05 & 0.01 & -2.84 & 39.19 & 0.048 & $0.74 \mathrm{E}+06$ \\
4.5 & 40.87 & 51.42 & 0.05 & 0.01 & -2.92 & 39.11 & 0.040 & $0.62 \mathrm{E}+06$ \\
5.0 & 40.77 & 51.32 & 0.05 & 0.01 & -3.02 & 39.01 & 0.032 & $0.49 \mathrm{E}+06$ \\
5.1 & 40.78 & 51.32 & 0.05 & 0.01 & -3.01 & 39.02 & 0.032 & $0.51 \mathrm{E}+06$ \\
5.2 & 40.49 & 51.05 & 0.01 & 0.00 & -3.29 & 38.75 & 0.017 & $0.26 \mathrm{E}+06$ \\
5.3 & 40.48 & 51.04 & 0.01 & 0.00 & -3.29 & 38.74 & 0.017 & $0.27 \mathrm{E}+06$ \\
5.4 & 40.39 & 50.96 & 0.01 & 0.00 & -3.38 & 38.66 & 0.014 & $0.21 \mathrm{E}+06$ \\
\hline
\end{tabular}

Table 20.b. Emission line spectrum at solar metallicity (I)

\begin{tabular}{cccccccc}
\hline Age & Ly $\alpha$ 1216 & HeII 1640 & MgII 2800 & [OII] 3727 & HeII 4686 & [OIII] 5007 & [OI] 6300 \\
\hline 1.5 & 22.88 & 0.00 & 0.02 & 1.75 & 0.00 & 0.50 & 0.02 \\
2.0 & 22.86 & 0.00 & 0.02 & 1.75 & 0.00 & 0.36 & 0.03 \\
2.5 & 22.86 & 0.00 & 0.02 & 1.74 & 0.00 & 0.38 & 0.02 \\
3.0 & 22.86 & 0.00 & 0.01 & 1.72 & 0.00 & 0.29 & 0.02 \\
3.5 & 22.87 & 0.00 & 0.01 & 1.67 & 0.00 & 0.08 & 0.03 \\
4.0 & 22.86 & 0.00 & 0.01 & 1.67 & 0.00 & 0.10 & 0.02 \\
4.5 & 22.87 & 0.00 & 0.01 & 1.67 & 0.00 & 0.08 & 0.03 \\
5.0 & 22.87 & 0.00 & 0.01 & 1.69 & 0.00 & 0.06 & 0.03 \\
5.1 & 22.87 & 0.00 & 0.01 & 1.69 & 0.00 & 0.06 & 0.03 \\
5.2 & 22.85 & 0.00 & 0.01 & 1.33 & 0.00 & 0.01 & 0.04 \\
5.3 & 22.85 & 0.00 & 0.01 & 1.35 & 0.00 & 0.01 & 0.04 \\
5.4 & 22.83 & 0.00 & 0.01 & 1.23 & 0.00 & 0.00 & 0.04 \\
\hline
\end{tabular}

Table 20.c. Emission line spectrum at solar metallicity (II)

\begin{tabular}{cccccccc}
\hline \hline Age & [NII] 6584 & [SII] 6716 & [SII] 6731 & [SIII] 9532 & [CII] 158 $\mu \mathrm{m}$ & $\mathrm{EW}(\mathrm{H} \beta)$ & $T_{e}(K)$ \\
\hline 1.5 & 0.85 & 0.35 & 0.24 & 0.73 & 1.42 & 113.46 & 6270 \\
2.0 & 0.93 & 0.38 & 0.26 & 0.70 & 1.65 & 100.72 & 6260 \\
2.5 & 0.91 & 0.37 & 0.26 & 0.70 & 1.62 & 100.75 & 6260 \\
3.0 & 0.94 & 0.38 & 0.26 & 0.68 & 1.76 & 95.94 & 6240 \\
3.5 & 1.02 & 0.42 & 0.29 & 0.61 & 2.15 & 83.91 & 6170 \\
4.0 & 1.01 & 0.39 & 0.27 & 0.63 & 2.09 & 90.88 & 6180 \\
4.5 & 1.02 & 0.44 & 0.30 & 0.58 & 2.15 & 70.34 & 6180 \\
5.0 & 1.04 & 0.50 & 0.34 & 0.53 & 2.18 & 54.38 & 6200 \\
5.1 & 1.04 & 0.49 & 0.34 & 0.54 & 2.18 & 54.68 & 6200 \\
5.2 & 0.91 & 0.59 & 0.41 & 0.29 & 2.38 & 28.50 & 5920 \\
5.3 & 0.92 & 0.60 & 0.41 & 0.30 & 2.38 & 27.41 & 5940 \\
5.4 & 0.87 & 0.61 & 0.42 & 0.23 & 2.39 & 22.47 & 5870 \\
\hline
\end{tabular}

Table 20.d. Emission line spectrum at twice solar metallicity (I)

\begin{tabular}{cccccccc}
\hline \hline Age & Ly $\alpha$ 1216 & HeII 1640 & MgII 2800 & [OII] 3727 & HeII 4686 & [OIII] 5007 & [OI] 6300 \\
\hline 1.5 & 22.39 & 0.00 & 0.00 & 0.55 & 0.00 & 0.07 & 0.02 \\
2.0 & 22.42 & 0.00 & 0.00 & 0.49 & 0.00 & 0.04 & 0.02 \\
2.5 & 22.41 & 0.00 & 0.00 & 0.48 & 0.00 & 0.05 & 0.02 \\
3.0 & 22.42 & 0.00 & 0.00 & 0.46 & 0.00 & 0.03 & 0.02 \\
3.5 & 22.47 & 0.00 & 0.00 & 0.40 & 0.00 & 0.01 & 0.02 \\
4.0 & 22.47 & 0.00 & 0.00 & 0.39 & 0.00 & 0.01 & 0.01 \\
4.5 & 22.48 & 0.00 & 0.00 & 0.39 & 0.00 & 0.01 & 0.02 \\
5.0 & 22.51 & 0.00 & 0.00 & 0.39 & 0.00 & 0.01 & 0.02 \\
5.1 & 22.52 & 0.00 & 0.00 & 0.39 & 0.00 & 0.01 & 0.02 \\
5.2 & 22.48 & 0.00 & 0.00 & 0.29 & 0.00 & 0.00 & 0.02 \\
5.3 & 22.48 & 0.00 & 0.00 & 0.29 & 0.00 & 0.00 & 0.02 \\
5.4 & 22.45 & 0.00 & 0.00 & 0.26 & 0.00 & 0.00 & 0.02 \\
\hline
\end{tabular}

Table 20.e. Emission line spectrum at twice solar metallicity (II)

\begin{tabular}{cccccccc}
\hline \hline Age & [NII] 6584 & [SII] 6716 & [SII] 6731 & [SIII] 9532 & [CII] 158 $\mu \mathrm{m}$ & $\left.\mathrm{EW}_{(\mathrm{H}} \beta\right)$ & $T_{e}(K)$ \\
\hline 1.5 & 0.49 & 0.23 & 0.16 & 0.40 & 2.33 & 116.06 & 4370 \\
2.0 & 0.49 & 0.24 & 0.16 & 0.38 & 2.67 & 103.09 & 4400 \\
2.5 & 0.48 & 0.23 & 0.16 & 0.38 & 2.62 & 103.12 & 4380 \\
3.0 & 0.49 & 0.23 & 0.16 & 0.37 & 2.82 & 98.34 & 4390 \\
3.5 & 0.47 & 0.24 & 0.16 & 0.34 & 3.43 & 85.68 & 4370 \\
4.0 & 0.46 & 0.22 & 0.15 & 0.35 & 3.33 & 93.07 & 4350 \\
4.5 & 0.47 & 0.25 & 0.17 & 0.33 & 3.44 & 71.73 & 4390 \\
5.0 & 0.50 & 0.28 & 0.19 & 0.31 & 3.53 & 55.26 & 4450 \\
5.1 & 0.49 & 0.28 & 0.19 & 0.32 & 3.52 & 55.56 & 4450 \\
5.2 & 0.40 & 0.29 & 0.20 & 0.16 & 3.80 & 28.89 & 4240 \\
5.3 & 0.41 & 0.30 & 0.21 & 0.16 & 3.81 & 27.85 & 4260 \\
5.4 & 0.37 & 0.29 & 0.20 & 0.12 & 3.85 & 22.61 & 4180 \\
\hline
\end{tabular}




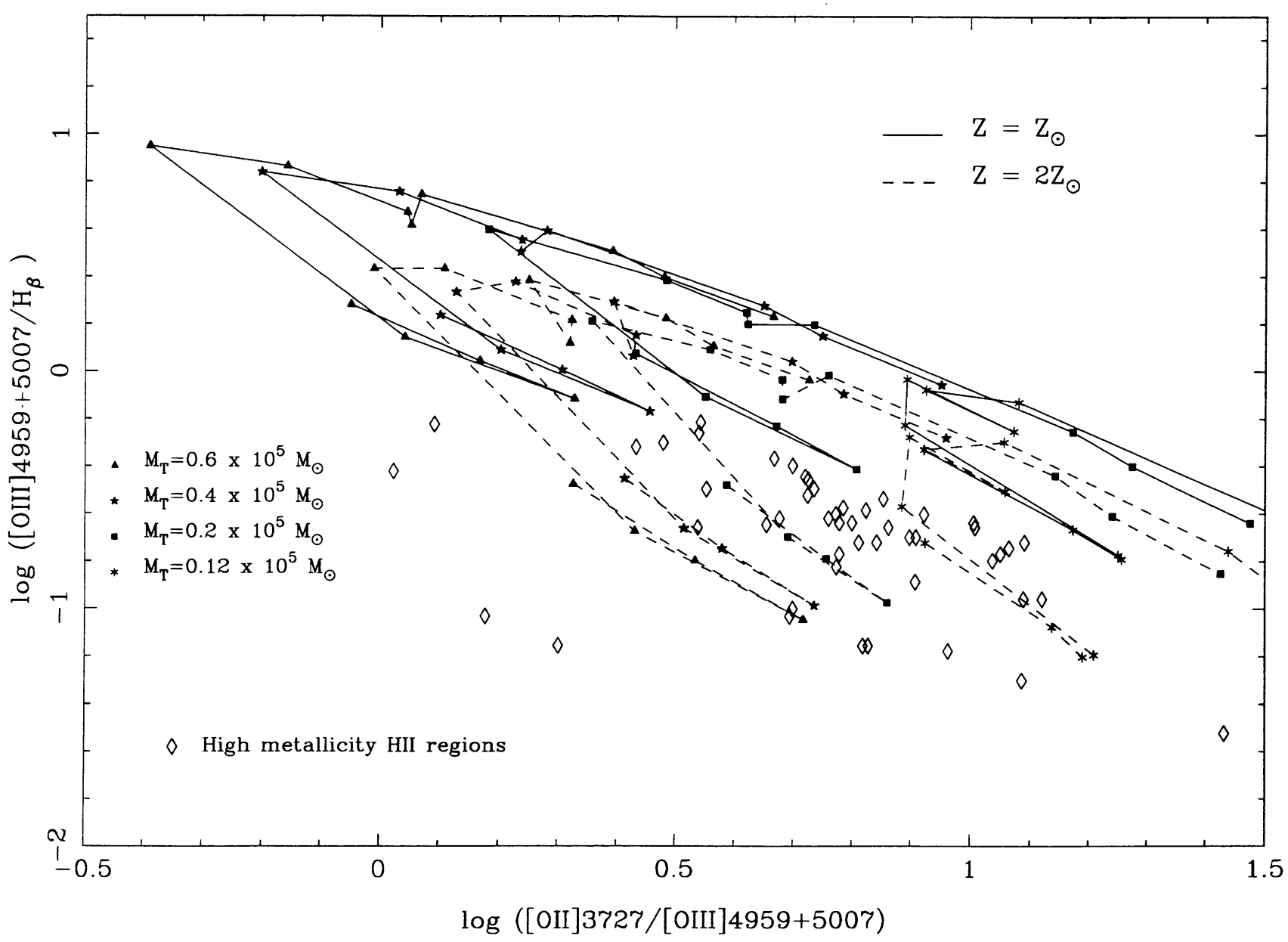

FIG. 5.-Diagnostic diagram. Clusters synthesized with the standard IMF. Different types of symbols represent the evolution of clusters of different masses, $M_{T}=0.12,0.2,0.4,0.6 \times 10^{5} M_{\odot}$. These points, representative of the ages of $1.5,2,2.5,3,3.5,4,4.5,5,5.1,5.2,5.3$, and 5.4 Myr, are joined with a solid line $\left(Z=Z_{\odot}\right)$ or a dashed line $\left(2 Z_{\odot}\right)$.

emitting regions would then correspond to different gas clouds with different physical properties. This case is worth exploring constructing models with a different geometry and matterbounded regions.

A second alternative, consistent with observations, is to assume that the ionizing cluster consists of smaller subclusters of different ages with the older ones providing the bulk of the continuum light and the younger ones dominating the ionization as has been previously suggested by Díaz et al. (1991). We have applied this approach to the case of NGC 604 in M33 and all the observables [ size, $u, n_{\mathrm{H}}$, emission-line ratios, $\mathrm{EW}(\mathrm{H} \beta)$, $\mathrm{H} \alpha$ luminosity, etc.] have been fitted satisfactorily by appropriate clusters synthesized with a standard IMF (Díaz \& García-Vargas 1994).

The model results concerning emission lines can be more easily examined by means of the diagnostic diagrams. Figure 5 shows an excitation diagram in which the value of $\log ([\mathrm{O} \mathrm{III]} / \mathrm{H} \beta)$ is plotted versus $\log ([\mathrm{O} \mathrm{II}] /[\mathrm{O} \mathrm{III}])$. Different types of symbols represent the evolution of clusters of different masses, $M_{T}=0.12,0.2,0.4,0.6 \times 10^{5} M_{\odot}$. These points, repre- sentative of the ages of $1.5,2,2.5,3,3.5,4,4.5,5,5.1,5.2,5.3$, and $5.4 \mathrm{Myr}$ are joined with a solid line $\left(Z_{\odot}\right)$ or a dashed line $\left(2 Z_{\odot}\right)$.

The observational data for extragalactic low-excitation (high-metallicity) H II regions are from Phillips et al. (1984); Díaz (1985); McCall, Rybski, \& Shields (1985); Vílchez et al. (1988); Díaz et al. (1990, 1991); and Henry et al. (1992). They are plotted as open diamonds in Figures 5-7. These data are located in a certain zone of the diagram, presenting some scatter, especially at the left part.

The lines representing the models show a sequence in the sense that points corresponding to the most massive clusters are in the high-excitation zone, while those corresponding to the less massive clusters are in the low-excitation zone. This effect corresponds to a sequence in ionization parameter. In fact, there is a good correlation between $M_{T}$ and $u$ as determined from the observed ratio [S II] 6716, 6731/[S III]9069, 9532 (Díaz et al. 1991). The age of the cluster, i.e., the shape of the ionizing radiation, affects the ionization parameter but to a lesser degree, compared with the effect of the mass. 


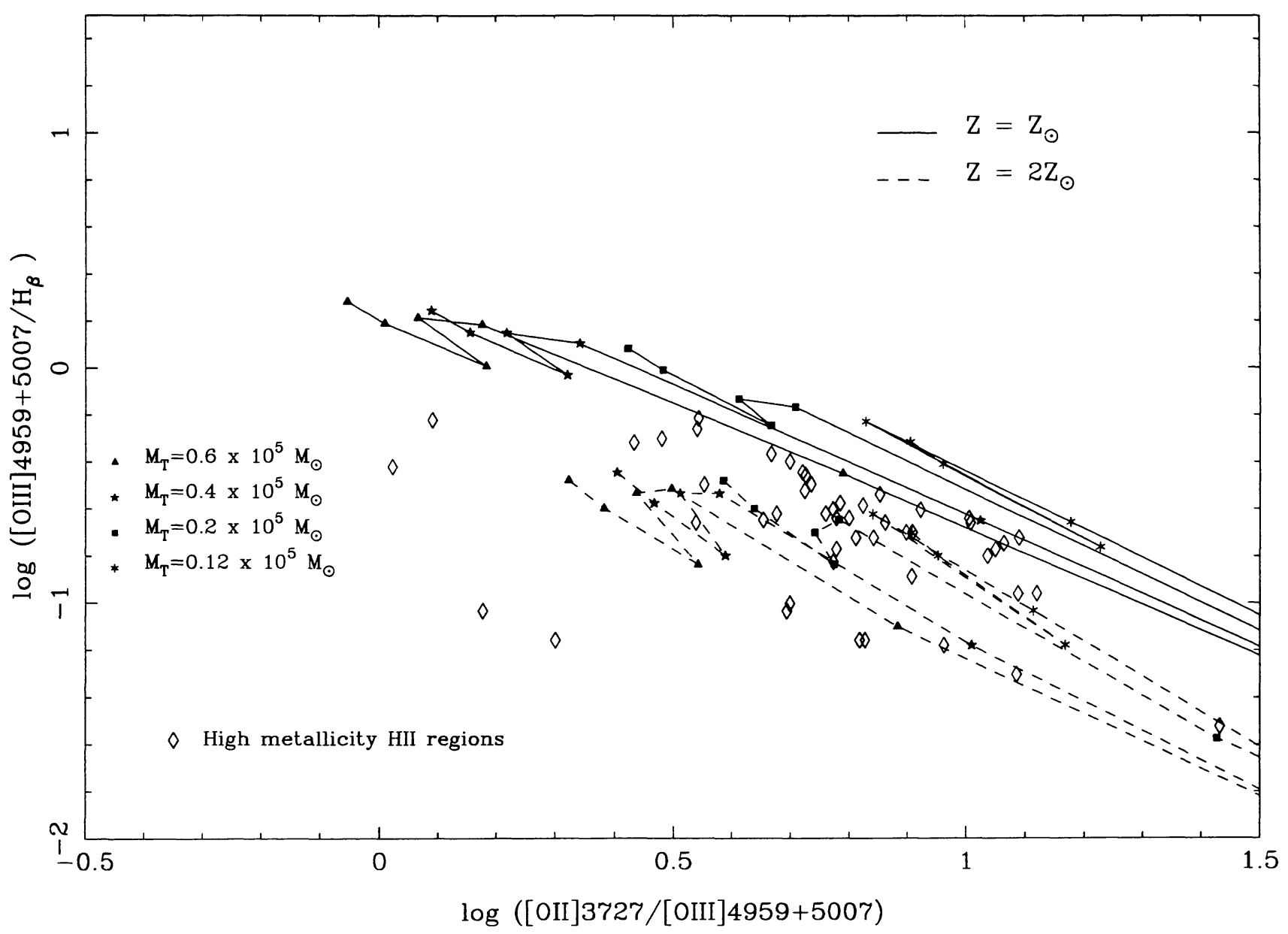

FIG. 6.-Diagnostic diagram. Clusters synthesized with the standard IMF and the stellar evolution given by Maeder (1990).

The effect of a higher metallicity is to move the points down and toward the right in the most massive clusters (high-excitation zone to the low-excitation zone); in the less massive clusters, the effect is to move down the points, but at the same level of ionization. One could think that this effect is due to the fact that the $T_{\text {eff }}$ of the ionizing radiation is a function of metallicity, in the sense that $T_{\text {eff }}$ of the radiation of a star cluster is lower when the metallicity increases as has been suggested by McGaugh (1991). However, in our set of models we have not included the evolutionary tracks or the atmosphere models at twice solar metallicity. The only difference between solar metallicity and twice solar metallicity models is the composition of the gas, which modifies the cooling efficiency predominantly through the oxygen lines.

One of the features of the diagnostic diagrams is the lack of observed data in the high-excitation region, predicted by theoretical models. This effect has been found before, as discussed in Vílchez et al. (1988), and recently, Díaz et al. (1991) have confirmed the existence of an inverse correlation between both ionization parameter and $T_{\text {eff }}$ of the ionizing radiation with metallicity. This could be due to different reasons. First, the evolutionary tracks we have used are not the correct ones, and the more massive stars do not evolve to extreme W-R. We have synthesized the stellar clusters using the new evolutionary tracks by Maeder (1990), in which the more massive stars do not evolve to the extreme $\mathrm{W}-\mathrm{R}$ phase, due to some considerations about the mass-loss rates and the opacity of the W-R winds. The results of these computations can be seen in Figure 6, which shows the same diagnostic diagram as Figure 5, but using clusters, synthesized with Maeder's (1990) stellar evolution, and a standard IMF. These models do not have hot W-R stars and therefore are restricted to the zone in which the $\mathrm{H}$ II regions are located. However, there are some observations which claim that these hot and luminous stars do exist (Davidson \& Kinman 1982; Barlow \& Hummer 1982; Garnett et al. 1991; Dopita et al. 1990), and they are not accounted for by this new stellar evolution. For this reason, we have not considered these evolutionary tracks.

Second, extreme W-R stars do exist and so also do the higher excitation regions but they have been excluded from the sample due to selection effects. In principle, one might think that if extreme W-R stars exist in GEHRs, they would be preferentially selected due to their high luminosity. However, according to our models, the $\mathrm{H} \alpha$ luminosity of a cluster with and 


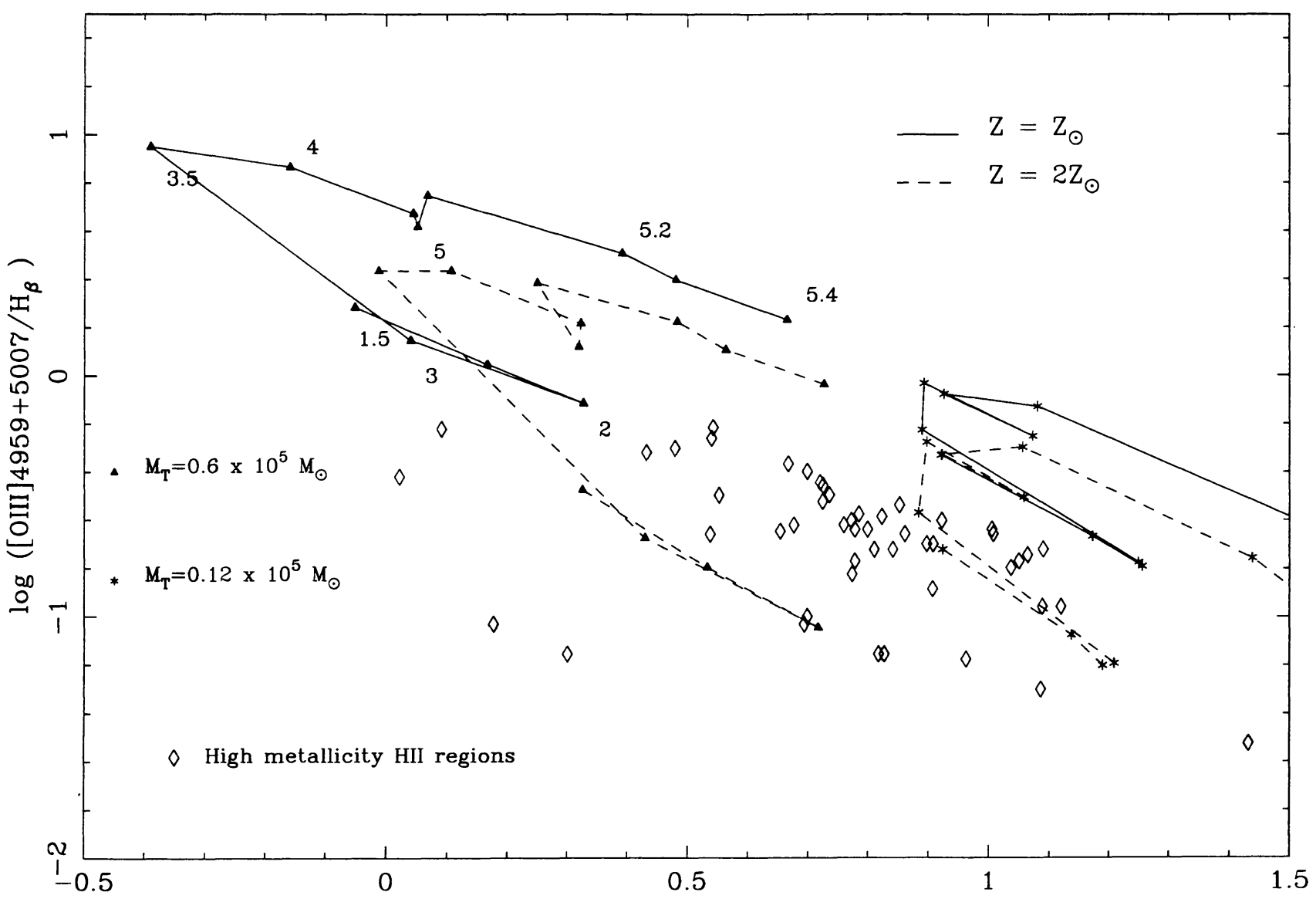

$\log ([\mathrm{OII}] 3727 /[\mathrm{OIII}] 4959+5007)$

FIG. $7 a$

FIG. 7.-( $a$ ) Diagnostic diagram. Standard IMF. $(b)$ Diagnostic diagram. $\alpha=3.35 m_{\mathrm{up}}=120 M_{\odot} .(c)$ Diagnostic diagram. $\alpha=3.35 m_{\mathrm{up}}=40 M_{\odot}$.

without extreme W-R stars is very similar, and most $\mathrm{H}$ II regions have been selected by their $\mathrm{H} \alpha$ luminosity. In fact, the probability of observing an $\mathrm{H}$ II region ionized by one or several of these stars should only depend on the probability of forming them in a given cluster, and this is intrinsically related to the way in which star formation proceeds. In our models we have considered that stars form with a mass spectrum given by an analytical law; this leads to a richness effect in the cluster, already mentioned in this paper, in the sense that very massive stars cannot form in small clusters. If the mass of the ionizing cluster is higher than $M_{\text {lim }}=1 \times 10^{4} M_{\odot}$, there should be some of these high-luminosity hot stars at some point during the evolution of the cluster. According to our models, a given cluster spends $1.9 \mathrm{Myr}$ out of a total of 5.4 Myr in which the $\mathrm{H}$ II region is observable, in this evolutionary phase. Therefore, about $35 \%$ of the observed high metallicity $\mathrm{H}$ II regions should be in the high-excitation zone, a probability high enough for detection. However, if GEHRs are preferentially ionized by a set of small star clusters, as has previously been suggested, this could be the reason that all observed high-metallicity $\mathrm{H}$ in regions have low excitation.

On the other hand, if star formation proceeds stochastically, there is always a probability of forming a very massive star even if the cluster mass is small, and consequently there is a probability of observing an $\mathrm{H}$ II region ionized by it. We have calculated the probability of forming very massive stars $(M>$ $60 M_{\odot}$ ) that will certainly become extreme and luminous W-R by performing Monte Carlo simulations. In clusters with masses higher than $6 \times 10^{3} M_{\odot}$ with a standard IMF, it is $100 \%$. This already lowers the value of $M_{\text {lim }}$. Moreover, the probability of forming one star with $M>60 M_{\odot}$ in clusters with masses as low as $0.6 \times 10^{3} M_{\odot}$ is in the range $35 \%-60 \%$. In this case, if the region is ionized by a set of different, small $\left(M<0.6 \times 10^{3} M_{\odot}\right)$ clusters, the probability of finding one of them with a star of mass higher than $60 M_{\odot}$ in the extreme $\mathrm{W}-\mathrm{R}$ phase is not zero as in the case of the analytical IMF, but 


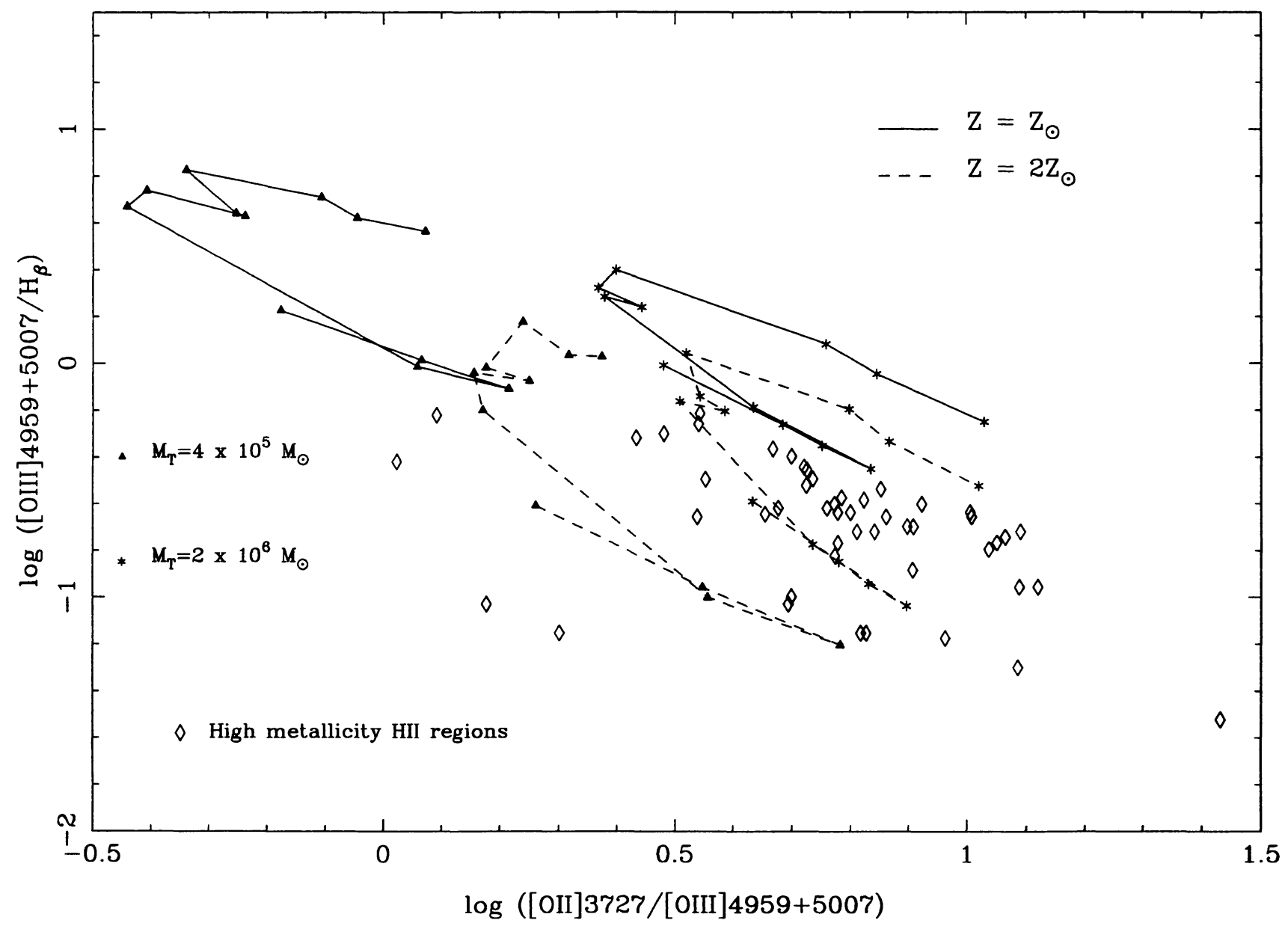

FIG. $7 b$

rises to $12 \%$. Our sample of $\mathrm{H}$ II regions is probably too small to extract any definite conclusion about the presence of these stars out of these figures.

The above discussion leads us to conclude that, if the ionizing clusters have masses greater than $0.6 \times 10^{3} M_{\odot}$, then the IMF parameters ( $\alpha$ and $m_{\mathrm{up}}$ ) have to be different from standard for regions of high metallicity. In fact, the existence of the inverse correlations ( $T_{\text {eff }}$ and $\log u ; T_{\text {eff }}$ and $Z$ ) mentioned above has been interpreted in this sense by different authors. Shields \& Tinsley (1976) suggested a change in the upper limit of the IMF, being higher at low metallicity; Terlevich \& Melnick (1981) suggested an increase in the slope of the IMF $(\alpha)$ with metallicity. This last approach has been used in other works (Campbell 1988; Copetti, Pastoriza, \& Dottori 1985a, b).

Figure $7 a$ shows the $\log ([\mathrm{O} \mathrm{II}] /[\mathrm{O} \mathrm{III}])$ versus $\log ([\mathrm{O} \mathrm{m}] /$ $\mathrm{H} \beta$ ) diagram from models corresponding to clusters with different masses synthesized with the standard IMF and $Z=Z_{\odot}$ (solid line), or $2 Z_{\odot}$ (dashed line). We have selected model clusters with two extreme masses to simplify the figure. Intermediate-mass clusters would fill the space between the two tracks in the plot. Figures $7 b$ and $7 c$ show the same diagnostic diagram as Figure $7 a$ but for clusters synthesized with $\alpha=$
3.35, $m_{\mathrm{up}}=120 M_{\odot}$, and clusters synthesized with $\alpha=3.35$, $m_{\text {up }}=40 M_{\odot}$. Stellar clusters which reproduce the observations have a high value of $\alpha(\simeq 3.35)$, a low value of $m_{\text {up }}$, and $Z \simeq 2 Z_{\odot}$. Therefore, our study would seem to indicate that both effects would be present, and that both $\alpha$ and $m_{\text {up }}$ would need to change with metallicity, with $\alpha$ increasing and $m_{\text {up }}$ decreasing with increasing metallicity.

Still there is some room for an IMF with standard parameter values if the regions are ionized by small star clusters. Spatially resolved observations of nearby GEHRs are then of great importance in order to decide this matter, as well as any independent method to determine the masses of the ionizing clusters.

\section{SUMMARY AND CONCLUSIONS}

The evolutionary models presented in this paper try to give a physical meaning to the functional parameters of giant extragalactic $\mathrm{H}$ II regions-ionization parameter, effective temperature of the ionizing radiation, and metallicity-assigning them to properties of the assumed ionizing clusters-mass, age, and metallicity, respectively.

We have considered clusters of stars of solar metallicity evolving in gaseous environments of metallicity solar or twice 


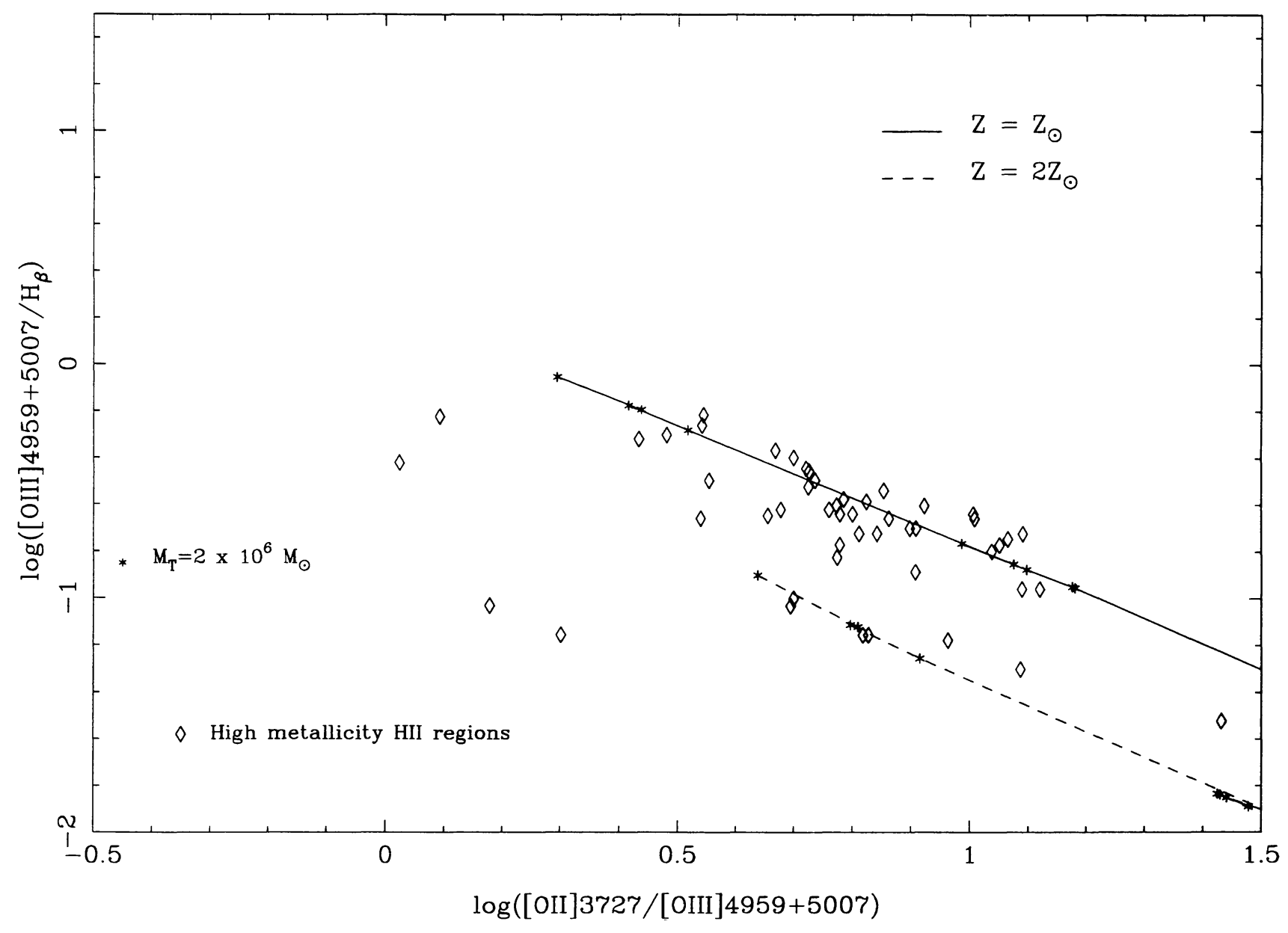

FIG. $7 c$

solar. One feature of these clusters is the appearance of extreme $\mathrm{W}-\mathrm{R}$ stars at some point during the evolution of the cluster. These stars, which have been observed in some occasions, if present would modify the high-energy end of the ionizing radiation, producing $\mathrm{H}$ II regions of high excitation. These regions apparently have not been observed.

This observational fact leads to the conclusion that if the ionizing clusters are relatively massive $\left(M>0.6 \times 10^{3} M_{\odot}\right)$, the IMF parameters have to be different from those derived for the solar neighborhood. In particular, the slope of the IMF should be higher and the upper mass limit should be lower than standard.

On the other hand, no changes to the IMF parameters would be required if the ionization is produced by a combination of ionizing continua coming from different small clusters. This second hypothesis would also explain the low equivalent widths of $\mathrm{H} \beta$ normally observed.

An exhaustive study of nearby GEHRs, including spatial resolution and determinations of the dynamical mass involved, is needed in order to decide among the different possibilities discussed here.

We thank Gary Ferland for kindly making available his code. We also thank Bernard Pagel, Roberto Terlevich, and Elena Terlevich for a critical reading of the manuscript and for many helpful comments and discussions. This work has been partially supported by the Spanish DGICYT project PB870080, and the Integrated Action with Great Britain 1989/198.

\section{REFERENCES}

Barlow, M. J., \& Hummer, D. G. 1982, in IAU Symp. 99, Wolf-Rayet Stars: Observations, Physics, Evolution, ed. C. W. H. de Loore \& A. J. Willis (Dordrecht: Reidel), 387

Binette, L. 1985, A\&A, 143, 334

Campbell, A. 1988, ApJ, 335, 644

Clegg, R. E. S., \& Middlemass, D. 1987, MNRAS, 228, 759
Copetti, M. V. F., Pastoriza, M. G., \& Dottori, H. 1985a, A\&A, 152, 427 1985b, A\&A, 156, 111

Davidson, K., \& Kinman, T. D. 1982, PASP, 94, 634

De Robertis, M. M., \& Osterbrock, D. E. 1986, ApJ, 301, 727

Díaz, A. I. 1985, PhD thesis, Sussex Univ.

Díaz, A. I., \& Garcia-Vargas, M. L. 1994, in preparation 
No. 2, 1994

GIANT EXTRAGALACTIC H II REGIONS

581

Díaz, A. I., Terlevich, E., Pagel, B. E. J., Vílchez, J. M., \& Edmunds, M. G. 1987, MNRAS, 226, 19

1990, in Chemical and Dynamical Evolution of Galaxies, ed. F. Ferrini, J. J. Franco, \& F. Matteucci, p. 239

Díaz, A. I., Terlevich, E., Vílchez, J. M., Pagel, B. E. J., \& Edmunds, M. G. 1991, MNRAS, 253, 245

Dopita, M., Lozinskaya, T., MacGregor, P., \& Rawlings, S. 1990, ApJ, 351,563

Ferland, G. J. 1990, HAZY: A Brief Introduction to CLOUDY, V.76.03, 1990 June

Ferland, G. J., \& Osterbrock, D. E. 1986, ApJ, 300, 658

Garnett, D., Kennicutt, R., Chu, Y., \& Skillman, E. 1991, ApJ, 373, 458

Grevesse, N., \& Anders, E. 1989, in Cosmic Abundances of Matter, ed. C. J. Waddington (AIP Conf. Proc. 183) (New York: AIP)

Henry, R. B. C., Pagel, B. E. J., Lasseter, D. F., \& Chincarini, G. L. 1992, MNRAS, 258, 321

Jacobi, G. H., Hunter, D. A., \& Christian, C. A. 1984, ApJs, 56, 257

Kennicutt, R. C. 1991, in Massive Stars in Starburst, ed. C. Leitherer, N. R. Walborn, T. M. Heckman, \& C. A. Norman, (Cambridge: Cambridge Univ. Press)

Kurucz, R. 1979, ApJS, 40, 1

Maeder, A. 1990, A\&AS, 84, 139
Maeder, A., \& Meynet, G. 1989, A\&A, 210, 55

Mas-Hesse, J. M., \& Kunth, D. 1991, A\&AS, 88, 399

Mathis, J. S., Chu, Y. H., \& Peterson, D. E. 1985, ApJ, 292, 155

McCall, M. C., Rybski, P. M., \& Shields, G. A. 1985, ApJS, 57, 1

McGaugh, S. S. 1991, ApJ, 380, 140

Melnick, J., Terlevich, R., \& Eggleton, P. P. 1985, MNRAS, 216, 255

Péquignot, D. 1984, A\&A, 131, 159

Phillips, M. M., Pagel, B. E. J., Edmunds, M. G., \& Díaz, A. I. 1984, MNRAS, 210,701

Shields, G. A. 1990, ARA\&A, 28, 525

Shields, G. A., \& Tinsley, B. M. 1976, ApJ, 203, 66

Skillmann, E. D. 1985, ApJ, 290, 449

Stasińska, G. 1984, A\&A, 135, 341 1990, A\&AS, 83, 501

Terlevich, R., \& Melnick, J. 1981, MNRAS, 195, 839

Terlevich, R., Tenorio-Tagle, G., Franco, J., \& Melnick, J. 1992, MNRAS, 255,713

van der Hulst, J. M., Kennicutt, R. C., Crane, P. C., \& Rots, A. H. 1988, A\&AS, 195, 38

Vílchez, J. M., Pagel, B. E. J., Díaz, A. I., Terlevich, E., \& Edmunds, M. G. 1988, MNRAS, 235, 633 